TITLE:

\title{
Neural mechanisms of dual-task interference and cognitive capacity limitation in the prefrontal cortex.
}

$\operatorname{AUTHOR}(\mathrm{S})$ :

Watanabe, Kei; Funahashi, Shintaro

\section{CITATION:}

Watanabe, Kei ...[et al]. Neural mechanisms of dual-task interference and cognitive capacity limitation in the prefrontal cortex.. Nature neuroscience 2014

ISSUE DATE:

2014-03-02

URL:

http://hdl.handle.net/2433/182955

\section{RIGHT:}

(C) 2014 Macmillan Publishers Limited.; 許諾条件により本文は2014-0903 に公開.; この論文は出版社版でありません。引用の際には出版社版 をご確認ご利用ください。; This is not the published version. Please cite only the published version. 


\title{
Neural mechanisms of dual-task interference and cognitive capacity limitation in prefrontal cortex
}

\author{
Kei Watanabe ${ }^{1,2,3} \&$ Shintaro Funahashi ${ }^{2 \dagger}$
}

1. Japan Society for the Promotion of Science (JSPS), Tokyo, 102-8472, Japan

2. Kokoro Research Center, Kyoto University, Kyoto, 606-8501, Japan

3. Present address: Department of Experimental Psychology, University of Oxford, OXI 3UD, $U K$

$†$ To whom correspondence should be addressed

Shintaro Funahashi, Ph.D.

Professor

Kokoro Research Center

Kyoto University

46 Shimoadachi-cho, Yoshida Sakyo-ku,

Kyoto, 606-8501, Japan

Phone \& Fax: (+81) 75-753-9674

E-mail: funahashi.shintaro.2z@kyoto-u.ac.jp 


\begin{abstract}
Simultaneous performance of two tasks often leads to performance deficits in the component tasks. This effect, known as dual-task interference, is thought to be a proof of capacity limitation in cognition, and the lateral prefrontal cortex (LPFC) has been highlighted as its putative neural substrate. Here, we recorded single-neuron activities in LPFC while monkeys performed dual-tasks that required the simultaneous performance of a varying-load spatial attention task and a spatial memory task. We found that the performance of the monkeys exhibited dual-task interference, and prefrontal neuron activities showed a decreased ability to represent task-relevant information to a degree proportional to the increased demand of the concurrent counterpart task. The locus of the interference was shown to originate in the simultaneous, overloaded recruitment of the same LPFC neural population by the two tasks. These results provide direct neurophysiological evidence for, and constraints to, psychological models of dual-task interference and capacity limitation.
\end{abstract}


Despite its remarkable flexibility, human cognitive information processing is severely capacity-limited ${ }^{1}$. When conflicting and interfering streams of information have to be processed simultaneously in dual-tasks (e.g., talking on a cell phone while driving ${ }^{2,3}$ ), there are evident behavioral signs of capacity overload, such as a decrease in percent correct rates and prolonged response times relative to those in the individual tasks themselves ${ }^{4}$. Since this effect, known as dual-task interference, is thought to be direct evidence of cognitive capacity limitation ${ }^{1,5}$, dual-task performance has been extensively studied in cognitive psychology ${ }^{4,6-8}$, and several important theories have been proposed.

In one such theory, Kahneman ${ }^{9}$ and others ${ }^{10,11}$ have proposed a concept of 'resource', which corresponds to the brain's task-general information processing capacity that is shareable across concurrent tasks in a graded manner. Presumably, the amount of available resource limits the amount of information that can be processed at a time, and dual-task interference could occur if two tasks accessing this finite resource exceed the total resource that is available. This theory is supported by neuroimaging studies that have reported overlapping activations in the lateral prefrontal cortex (LPFC) between dual-tasks and single-tasks ${ }^{12-14}$. Based on the observations that dual-tasks activate overlapping regions of the LPFC to a greater degree than individual tasks performed separately, these studies suggested that the source of the interference and performance limits in dual-tasks resides in the competition for processing resources between the two concurrent tasks, and that LPFC is a likely substrate for this competition ${ }^{15,16}$. In other variants of this view, limitations in, or malfunctioning of, some task-general factors, such as of the central executive which is responsible for monitoring and coordinating concurrent-task processing, has been proposed as the source of dual-task interference ${ }^{6,17,18}$. Although these and other $^{19,20}$ earlier studies consistently proposed that the LPFC plays a key role in dual-task performance and interference, the underlying neuronal mechanisms remain largely unknown due to the lack of neurophysiological investigations, and several fundamental questions remain unanswered. 
First, it is not known whether dual-task performance affects the activities of LPFC neurons in a manner that could account for the behavioral dual-task interference effect. Second, it has not yet been shown whether the overlap of activity between the two concurrent tasks, which reflects the competition for processing resources, can be observed at the single-neuron level. Third, the neural mechanisms that underlie hypothetical task-general factors, such as cognitive resources, are almost completely unknown.

In this study, we first established a nonhuman primate model of dual-task interference to address these issues at the level of single prefrontal neurons. For the two component tasks in the present dual-task, we selected a visuo-spatial attention task and a visuo-spatial working memory task, since these tasks are known to require intact LPFC functioning ${ }^{21-23}$ by recruiting

the activation of many LPFC neurons ${ }^{24-27}$. We expected that the simultaneous performance of these two LPFC-demanding tasks would cause an interference effect, since the monkeys would be required to engage in the processing of two streams of task information that were overlapped with respect to both time and processing modality. We demonstrated that monkeys were capable of performing this dual-task and that their dual-task performance exhibited an interference effect similar to that in humans. At the neural level, we found that, during the dual-task, LPFC neuron activities exhibited a remarkable attenuation of selectivity for task content (i.e., spatial location), even in correct trials. The degree of this effect was directly proportional to the demand of the concurrent counterpart task. These results indicate that signs of dual-task interference are already manifest at the level of single-neuron activity in LPFC.

\section{RESULTS}

\section{Dual-task paradigm}

Two monkeys were trained in two versions of dual-tasks, standard and easy dual-tasks, performed in separate blocks. Both versions required the simultaneous performance of a spatial 
attention task and a spatial memory task, each of which engaged an independent behavioral response modality (lever-release and saccade, respectively).

Trials for the standard dual-task started with the attention task component (Fig. 1a), which was initiated by the monkey's lever-press together with presentation of a central fixation ring (FR). Two peripheral (Up and Down) rings (Fig. 1b) were presented $1.0 \mathrm{~s}$ after the start of fixation. Subsequently, an attention cue (red filled circle, $0.4 \mathrm{~s}$ ) was presented on one of the three rings to indicate the target ring for the current trial. The position of the attention cue corresponded to different attention conditions: the (Attend) Up, Down, and FR $\mathrm{Ftandard(std)}_{\text {( }}$ conditions. At 2.0-5.0 s from attention cue offset (Wait1 period), in $60 \%$ of the trials (Short trials), the color of the target ring turned to red ( $T_{\text {col }}$ change, $0.4 \mathrm{~s}$ ), and the monkeys were required to release the lever within $0.6 \mathrm{~s}$ (Lever-release). In the remaining $40 \%$ of the trials (Long trials), termination of the wait 1 period led to a change in the color of one of the two non-target rings (Catch change), to which the monkeys were prohibited from responding. The monkeys waited for another $0.4-3.4 \mathrm{~s}$ from the catch change offset (Wait2 period) before $\mathrm{T}_{\text {col }}$ change.

The memory task was added to the attention task (Fig. 1c,d; Memory cue) by the presentation of a memory cue $(0.4 \mathrm{~s})$ in one of five far-peripheral locations (Fig. 1e, black filled squares) at a random timing between attention cue offset and $\mathrm{T}_{\text {col }}$ change onset (1.6-5.1 $\mathrm{s}$ from attention cue offset). The monkeys were required to memorize this location while performing the attention task. At the end of the attention task, after lever-release and the subsequent follow-up fixation period $(0.4 \mathrm{~s})$, all of the rings disappeared and small place-holders were presented at five possible memory cue locations. The monkeys were required to make a saccade within $0.6 \mathrm{~s}$ to the location where the memory cue had been presented and to keep gazing at it for $0.6 \mathrm{~s}$ (Fig. 1c,d; Saccade). Importantly, memory cue presentation was scheduled independently of the attention task, and presentations scheduled after $\mathrm{T}_{\text {col }}$ change onset never occurred. Thus, a memory cue was presented in two-thirds of attention task trials, which made 
these trials a dual-task. The remaining one-third of trials were performed as a single attention task.

The event sequence of the easy dual-task was exactly the same as in the standard dual-task. However, the Up and Down rings were never presented and the attention cue was always presented on FR (the $\mathrm{FR}_{\text {easy }}$ condition). Catch change was scheduled but executed as an 'empty event' without any actual changes in the display items. Therefore, the identical memory task was performed concurrently (dually) with four different attention task conditions (Up, Down, $\left.\mathrm{FR}_{\text {std }}, \mathrm{FR}_{\text {easy }}\right)$. The difficulty levels of these four attention task conditions were expected to be different, which would lead to different degrees of interference in performance of the dually-performed memory task (DMT) (hereafter, the four DMT conditions are referred to as

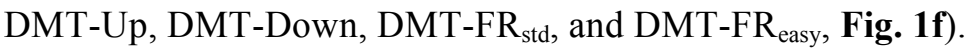

As a control condition, the monkeys performed the single memory task (SMT). The time course of the event sequence in SMT was the same as that in DMT; all attention task events were scheduled but executed as empty events without any physical stimulus change. To precisely determine the spatial selectivity of neuron activity, eight memory cue locations that covered both visual hemifields were used (Fig. 1e). Up and Down rings were presented to match the ring stimuli layout in the standard dual-task. During daily recording sessions, either the standard or easy dual-task was performed as a DMT block. Two SMT blocks, $\mathrm{SMT}_{\text {pre }}$ and $\mathrm{SMT}_{\text {post }}$, were performed before and after the DMT block, respectively. In the following analyses that compared behavior and neuronal activities across the SMT and DMT conditions, only the five memory cue locations that were used in both conditions were considered. The event sequence of example trials for the standard and easy dual-tasks, and SMT is shown in

\section{Supplementary Figure 1.}

\section{Behavioral performance}


An analysis of attention task performance showed that both monkeys exhibited different percent correct rates and response times (RT) across the four attention task conditions (Fig. 2a,b). In both monkeys, a series of six pairwise statistical comparisons (see Online Methods) for the percent correct rate and RT confirmed that the Up and Down conditions were more difficult than $\mathrm{FR}_{\text {std }}$ and $\mathrm{FR}_{\text {easy }}$; the former two showed significantly lower percent correct rates and significantly longer RTs than the latter two. In addition, $\mathrm{FR}_{\text {std }}$ was more difficult than $\mathrm{FR}_{\text {easy }}$; the former showed a significantly lower percent correct rate and, albeit only in monkey S, a moderately longer RT than the latter.

An analysis of memory task performance revealed that both monkeys exhibited lower percent correct rates in the DMT conditions compared with SMT (Fig. 2c). A series of 10 pairwise comparisons (see Online Methods) across the SMT and four DMT conditions confirmed the presence of significant dual-task interference in all four DMT conditions in monkey S and in three DMT conditions (DMT-Up, DMT-Down and DMT-FR std $_{\text {) }}$ in monkey A. The order of the performance level across the DMT conditions indicated that, in both monkeys, memory task performance was interfered with by the concurrent attention task to a degree proportional to the difficulty of the attention task. There was no common, systematic trend in changes in the percent correct rates across DMT-Up and DMT-Down as a function of the spatial proximity between the attention and memory cues (Fig. 2d,e). In monkey S, although the percent correct rates in both DMT-Up and DMT-Down tended to decrease as the spatial proximity between the two cues increased, these trends were not statistically significant (DMT-Up, $L=1247, P=0.12$; DMT-Down, $L=1235, P=0.30$; one-tailed Page's $L$ test). In monkey A, significant, but opposite, monotonic trends were observed in DMT-Up (increasing trend, $L=1430, P=0.002$ ) and DMT-Down (decreasing trend, $L=1417.5, P=0.003$ ). This suggests that the present dual-task effect cannot be explained by a spatial modality-specific effect, such as a chunking or crowding effect (Fig. 2d, right), and instead is strongly associated with the demands of the concurrent attention task. Additionally, in both monkeys, we found that 
a longer memory delay-period was associated with a greater magnitude of dual-task interference in DMT performance (Supplementary Fig. 2a); monkey A, whose overall DMT performance exhibited moderate interference compared with monkey S, also showed a highly significant interference effect in trials with a longer delay-period $(>4.0 \mathrm{~s})$.

Albeit only in monkey $\mathrm{S}$, we found signs of dual-task interference among fixation break (FB) errors that occurred following memory cue onset; as the concurrent attention task became more difficult, the oculomotor aspect of the memory task performance was interfered with to a greater extent (Supplementary Fig. 2b-d).

In separate sessions, we tested the reproducibility of the present effect, using a modified standard dual-task, in which non-cued trials were randomly intermixed (50\% of trials) among the existing three attention conditions of the standard dual-task (Up, Down, FR std; cued trials) (Supplementary Fig. 3a). In non-cued trials, all three of the rings were illuminated simultaneously by red filled circles in the attention cue period. A highly similar pattern of dual-task interference was replicated in this task, which demonstrated the robustness of the dual-task interference effect in the present spatial dual-task paradigm (Supplementary Fig. 3bd). Furthermore, the comparison of attention task performance between the cued and non-cued trials showed advantageous effects of cueing (higher percent correct rates and shorter lever-release RTs in cued trials), indicating that, in the standard dual-task, the monkeys maintained covert attention to a target ring that had been cued by an attention cue (Supplementary Fig. 3e,f). Admittedly, this does not mean that memory is not required in cued trials of the attention task.

\section{Single-neuron activity}

We recorded 160 single-neuron activities in the LPFC (Fig. 1g) of the two monkeys. To investigate how the performance of the present spatial dual-tasks affected LPFC single-neuron activities related to memory task performance, we compared spatially-selective cue- and 
delay-period activities across the SMT and DMT conditions. In the DMT conditions, the trials in which attention task events (i.e., catch change and $\mathrm{T}_{\text {col }}$ change) were presented immediately before (up to $-0.4 \mathrm{~s}$ ) or during each of these periods were excluded from the analysis. Activities were analyzed for correct trials.

Many LPFC neurons exhibited spatially-selective cue- (Fig. 3a) or delay-period activity (Fig. 4a,b) in $\mathrm{SMT}_{\text {pre }}$. However, the spatial selectivity of these activities was

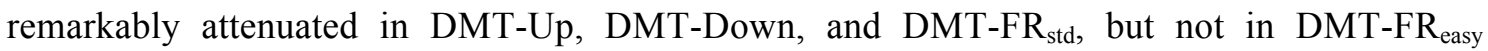
(cue-period activity, Fig. 3b-e; delay-period activity, Fig. 4a,b), indicating that dual-task performance affected the activities of LPFC neurons by reducing their spatial selectivity to a degree proportional to the difficulty of the concurrent attention task condition. Importantly, activities of the same neurons in $\mathrm{SMT}_{\text {post }}$ exhibited robust responses with highly significant spatial selectivity comparable to that in $\mathrm{SMT}_{\text {pre }}$ (cue-period activity, Fig. 3f; delay-period activity, Fig. $\mathbf{4 a}, \mathbf{b})$, suggesting that the attenuation of spatial selectivity in DMT was caused by the addition of the concurrent attention task, and cannot be attributed to other factors such as degradation in recording quality or changes in the neuron's intrinsic response properties. The same pattern was observed in other neurons (Supplementary Figs. 4 and 5), which further supports this view.

\section{Population analyses}

Among 91 neurons that showed spatially-selective cue-period activity in $\mathrm{SMT}_{\text {pre }}$, the activities of 61 and 37 were recorded in the standard and easy dual-tasks, respectively (7 recorded in both), and the activities of 73 were recorded in $\mathrm{SMT}_{\text {post. }}$ Of 71 neurons that had spatially-selective delay-period activity in $\mathrm{SMT}_{\text {pre }}, 51$ and 24 were recorded in the standard and easy dual-tasks, respectively (4 recorded in both), and 55 were recorded in $\mathrm{SMT}_{\text {post. }}$.

Figure 5a shows population cue-period activities across the six memory task conditions. Compared with $\mathrm{SMT}_{\text {pre}}$, the difference in activity between the maximum and 
minimum response locations was remarkably attenuated in DMT-Up, DMT-Down and DMT-FR $_{\text {std }}$, but not in DMT-FR easy, which demonstrates that the difficulty of the concurrent attention task was directly associated with the strength of the attenuation of spatial selectivity among cue-period activities (main effect of Task condition, $F_{5,378}=5.41, P=10^{-4}$; Cue location, $F_{1,378}=258.58, P<10^{-4}$; interaction, $F_{5,378}=11.12, P<10^{-4}$; two-way mixed-design ANOVA) A similar pattern was observed in population delay-period activity (Fig. 5c, main effect of Cue location, $F_{1,297}=132.27, P<10^{-4}$; interaction, $\left.F_{5,297}=8.72, P<10^{-4}\right)$. To further characterize this effect, we constructed a spatial tuning plot using each neuron's mean discharge rate in all five of the memory cue locations that were used in both the SMT and DMT conditions (see Online Methods). For both cue- (Fig. 5b) and delay-period activities (Fig. 5d), the regression slopes of the spatial tuning plots for DMT-Up, DMT-Down and DMT-FR std $_{\text {were significantly }}$ smaller than that for $\mathrm{SMT}_{\text {pre }}$ (cue-period activity: for all comparisons, $P<3 \times 10^{-4}$; delay-period activity: DMT-Up, $P=0.003$; DMT-Down, $P=0.001$; DMT-FR std $P=0.01$; test on equality of regression slopes, $p$-values adjusted by Holm's SRB procedure). However, there was no apparent degradation of spatial tuning in DMT-FR easy (cue-period activity, $P=0.18$; delay-period activity, $P=0.42$ ) or $\mathrm{SMT}_{\text {post }}$ (cue-period activity, $P=0.53$; delay-period activity, $P=0.71)$. In both monkeys, the spatial selectivity of cue- and delay-period activities was commonly attenuated under the DMT conditions (Supplementary Figs. 6 and 7). Qualitatively identical results were obtained after the normalized transformation of raw spike-rate data (each neuron's activity was divided by its peak cue- or delay-period activity in the maximum response location in $\mathrm{SMT}_{\text {pre }}$ ). A separate analysis confirmed that the neurons recorded in the standard dual-task (DMT-Up, DMT-Down and DMT-FR std $_{\text {) }}$ and the easy dual-task (DMT-FR easy) exhibited highly similar activities in the control SMT condition (Supplementary Fig. 8).

To further confirm the present neuronal effects, we quantified the strength of selectivity for the memory cue location in each neuron using a proportion of explained variance (PEV) as measured by $\omega^{2}$ (Supplementary Fig. 9). We found that the PEV values in DMT-Up, 


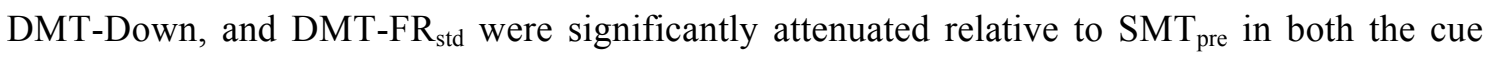
(Supplementary Fig. 9a,c) and delay periods (Supplementary Fig. 9b,d), in which a significant majority of spatially-selective neurons in $\mathrm{SMT}_{\text {pre }}$ showed a decrease in the PEV value under each of the three DMT conditions (binomial test, $P<0.05$ ). However, this was not the case for DMT-FR easy or $\mathrm{SMT}_{\text {post }}$. These results demonstrate that the significant behavioral cost of dual-task performance is already manifest at the level of LPFC single-neuron activity as the deficit in spatial information processing for memory cue location, which scales with the difficulty of the concurrent attention task.

\section{Neural interaction between the attention and memory tasks}

In DMT-Up and DMT-Down, the attenuation of spatial selectivity among cue- and delay-period activities could be explained within the framework of the attentional modulation of neural activity $^{28,29}$, in which the strength of modulation depends on the distance between a stimulus and the current locus of covert attention. For example, a neuron that exhibited maximum cue-period activity at the $90^{\circ}$ location in $\mathrm{SMT}_{\text {pre }}$ should show different cue-period activities between DMT-Up and DMT-Down, since in DMT-Up, the attention target ring was located near the neuron's maximum response location (congruent trials), whereas in DMT-Down, the target ring was located opposite from the maximum response location (incongruent trials). We found that both the magnitudes of cue- and delay-period activities at the maximum response location and the strength of spatial tuning did not differ between congruent and incongruent trials (cue-period activity: magnitude, $P=0.56$, Fig. 6a; tuning slope, $P=0.45$, Fig. $6 \mathbf{c}$; delay-period activity: magnitude, $P=0.23$, Fig. 6b; tuning slope, $P=0.95$, Fig. 6d). This indicates that the attenuation of spatial selectivity in DMT conditions cannot be explained by attentional modulation.

Another spatial factor that could have affected the cue- and delay-period activities in DMT is the difference in the number of ring stimuli on the monitor (i.e., 3-ring layout in the 
standard dual-task vs. 1-ring layout in the easy dual-task). In a separate experiment, using a modified single memory task in which these two layouts were randomly intermixed, we observed almost identical cue- (Supplementary Fig. 10a,b) and delay-period activities (Supplementary Fig. 10c) for the two layouts. This suggests that the presence or absence of two peripheral rings is unlikely to have affected the neuronal activities during dual-task performance via sensory-level lateral inhibition.

To determine whether the memory and attention tasks recruited the same LPFC neural population, we examined how neurons that exhibited spatially-selective activities in the single memory task responded during attention task events (Fig. 7). During the standard dual-task, the neurons that exhibited spatially-selective cue-period activity in $\mathrm{SMT}_{\text {pre }}$ showed significant spatial selectivity during the attention cue period (0.1-0.5 s from attention cue onset, Fig. 7a). Most neurons $(70 \%, 43 / 61)$ exhibited significant spatial selectivity for the attention cue location (Kruskal-Wallis test, $P<0.05$ ). This proportion reached $90 \%$ if we included neurons that did not show spatial selectivity but which did exhibit significantly modulated attention cue period activity compared with the baseline activity level ( $-0.4-0$ s from attention cue onset, Wilcoxon signed-rank test, $P<0.05)$ in at least one of three attention conditions $(20 \%)$. Similar results were obtained for the attention delay period activity (0-1.0 s from attention cue offset) (Fig. 7b). This indicates that the two component tasks in the standard dual-task recruited a remarkably overlapping neural population in the LPFC. Conversely, during the $\mathrm{FR}_{\text {easy }}$ condition in the easy dual-task, less than half of the neurons that exhibited spatially-selective cue- or delay-period activities in $\mathrm{SMT}_{\text {pre }}$ were recruited in the attention task by exhibiting a significant change in activity relative to the baseline (attention cue period, 46\%, 17/37, Fig. 7c; attention delay period $25 \%, 6 / 24$, Fig. 7d).

To directly compare the degree of recruitment across the four attention task conditions, we calculated the proportion of neurons that showed significant activity modulation relative to the baseline during the attention cue (Fig. 7e, left) and delay periods (Fig. 7e, right). For both 
periods, a significant difference in this proportion was observed across the four conditions (Extended Fisher's exact test, $P<0.05$ ), with $\mathrm{FR}_{\text {easy }}$ showing the smallest proportion. This suggests that, in the LPFC neural population, competition occurred for processing resources between the attention and memory tasks; as the difficulty of the attention task increased (Fig. 2a,b), processing in the attention task became more demanding, which left less processing ability available for the memory task, and thus elicited a loss of spatial-selectivity for memory cue encoding. Notably, during the attention delay period, in all three attention task conditions in the standard dual-task, a substantially higher proportion of inhibitory modulation was observed compared with $\mathrm{FR}_{\text {esay }}$ (Fisher's exact test, $P<0.09$, uncorrected; Fig. 7e, right). This trend further continued after the earliest possible timing of memory cue presentation (1-s time epoch after the vertical arrow in Fig. $\mathbf{7 b}, \mathbf{d}$ ). The proportions of neurons that exhibited inhibitory modulation in this time epoch were $24 \%, 18 \%, 14 \%$ and $4 \%$ in the Up, Down, $\mathrm{FR}_{\text {std }}$ and $\mathrm{FR}_{\text {easy }}$ conditions, respectively.

\section{Neural mechanism of adaptive cognitive capacity allocation}

To gain insight into the temporal dynamics of the competitive interaction between the attention and memory tasks in the standard dual-task, we examined how the neural signals encoding the information of each task evolved during a trial, using a partial $\omega^{2}$ PEV measure (Fig. 8a; see Online Methods). After attention cue onset, $\mathrm{PEV}_{\text {attention }}$ values exhibited a sustained elevation which continued until the conclusion of the attention task. Subsequently, presentation of the memory cue triggered the onset of $\mathrm{PEV}_{\text {memory }}$ and $\mathrm{PEV}_{\text {interaction }}$ components. However, the strength of $P E V_{\text {memory }}$ during the delay-period ("D" in Fig. 8a) was significantly attenuated relative to $\mathrm{SMT}_{\text {pre }}$ (dashed cyan line) (Wilcoxon signed-rank test, $P=3 \times 10^{-4}$ ). The concurrent elevation of the $\mathrm{PEV}_{\text {attention }}$ and $\mathrm{PEV}_{\text {memory }}$ components suggests that the information-processing capacities of LPFC task-related neurons are divided and allocated to the two tasks on an as-needed basis ${ }^{15,16}$. 
Another notable feature is that a significant reawakening of $\mathrm{PEV}_{\text {memory }}$ was observed after the conclusion of the attention task (see also Supplementary Figs. 5 and 11 for single-neuron examples). The $\mathrm{PEV}_{\text {memory }}$ in the follow-up fixation period (F) was significantly greater than that in the pre- $\mathrm{T}_{\text {col }}$ change period $(\mathrm{P})\left(\right.$ Fig. 8 b, $\left.P=2 \times 10^{-4}\right)$. Correspondingly, the weight given to the processing of each task changed dramatically between before and after the conclusion of the attention task. In the pre- $\mathrm{T}_{\mathrm{col}}$ change period, the processing of attention and memory task information were given equal weight $\left(\mathrm{PEV}_{\text {attention }}\right.$ vs. $\left.\mathrm{PEV}_{\text {memory }}, P=0.34\right)$, whereas in the follow-up fixation period, memory task information was more strongly represented than attention task information (Fig. 8c, $P=3 \times 10^{-4}$ ). Furthermore, although $\mathrm{PEV}_{\text {memory }}$ in the standard dual-task was elevated to a level comparable to that in $\mathrm{SMT}_{\text {pre }}$ in the follow-up fixation period (Fig. 8d, $P=0.72$ ), signs of neuronal dual-task interference were still evident in the saccade period (S); the $\mathrm{PEV}_{\text {memory }}$ in the standard dual-task was moderately attenuated compared to that in $\operatorname{SMT}_{\text {pre }}(P=0.12)$, with the clear absence of a phasic peak of PEV values which was associated with saccade execution in $\mathrm{SMT}_{\text {pre }}($ Fig. 8e, $P=0.001)$. This indicates that, in the standard dual-task, the reawakened spatial mnemonic information was still not fully linked to the output of motor command within the memory-based sensorimotor transformation loop in the LPFC. Similar results were observed in the time courses of the proportion of neurons with significant effects $(P<0.05)$ in each factor (Fig. 8f). In the easy dual-task (Supplementary Fig. 11b-d), the time course of $\mathrm{PEV}_{\text {memory }}$ showed an even more prominent reawakening phenomenon.

A separate analysis confirmed that these distinct patterns of $\mathrm{PEV}_{\text {memory }}$ time series observed in the present dual-tasks, which were characterized by initial attenuation and subsequent reawakening, are strongly associated with the presence of the processing demands for the concurrent attention task performance, rather than being attributable to a general, task-independent effect of task-difficulty (Supplementary Fig. 12). 
These results demonstrate that the reawakening of spatial selectivity for memory cue that occurred in concert with the reprioritization of task processing between the two concurrent tasks reflects the process in which the information-processing capacities of LPFC neurons are flexibly reallocated between the two tasks according to their current processing demands. We suggest that this reallocation of processing-capacity to the memory task compensated for the drastic loss of memory cue information in the earlier cue and delay periods, and thus underpinned the accuracy of DMT performance, the reduction of which relative to $\mathrm{SMT}_{\text {pre }}$ was still relatively moderate despite the drastic deterioration in neuronal memory task processing.

\section{DISCUSSION}

Dual-task interference is a classic behavioral demonstration of cognitive capacity limitation. This study reports two main findings that should contribute to the understanding of the neural mechanisms that underlie dual-task interference and capacity limitation. First, we showed that monkeys exhibited the classic pattern of behavioral interference that scales with task-difficulty; as the attention task becomes more difficult, the performance in the concurrent memory task decreases. This inverse relationship between the difficulty of one task and performance in the other is comparable to the results widely observed in previous human dual-task studies ${ }^{3,4,30}$, which demonstrates that, like humans, monkeys have sufficient but capacity-limited information-processing ability to perform dual-tasks.

Second, we demonstrated that dual-task interference was observed at the level of single-neuron activities in the LPFC. Selectivity was reduced among the memory cue- and delay-period activities to a degree proportional to the difficulty of the concurrent attention task. Importantly, a substantially overlapping portion of the neural population that engaged in memory task processing was also activated by the concurrent attention task, and there was a higher degree of recruitment by more difficult attention task conditions. Thus, the limited spatial processing abilities of LPFC neurons are more thinly spread across concurrent tasks as the task 
difficulty increases. Furthermore, we showed that these neural effects are unlikely to be caused by factors other than the interference caused by the demands of the performance of the concurrent attention task, such as changes in the neurons' intrinsic response properties or attentional modulation. Finally, after the conclusion of the attention task, the reduced selectivity for memory cue location showed significant reawakening. Taken together, these results identify the locus of dual-task interference as the competitive, overloaded recruitment of an overlapping LPFC neural population by two concurrent tasks, as postulated in the previous neuroimaging studies $^{15,16}$. The observed pattern of the neuronal dual-task interference effect suggests that the information-processing capacity of single-neurons in the LPFC (1) is limited to a fixed level, below which the information in two concurrent tasks can be fully accommodated, (2) is flexibly allocated and reallocated among two tasks on an as-needed basis, and (3) enhances behavioral performance as its allocation to one task increases. These characteristics agree well with the functional characteristics of cognitive resource that have been postulated in the resource theory of dual-task interference ${ }^{9-11}$, and with a recent model of flexible resource allocation in visual attention and visual working memory ${ }^{31}$.

A prior study reported that a mutually inhibitory network between spatially-selective neurons with different spatial selectivities contributes to the enhancement of spatial selectivity in LPFC delay-period activities ${ }^{32}$. We suggest that in the present dual-tasks that used spatial cueing as a common input modality, the preemption of this inhibitory network by the attention task, which occurred to a degree proportional to the task difficulty (Fig. 7e, right), led to differential attenuation in the shaping of spatial selectivity among delay-period activities in the memory task. Therefore, the competitive recruitment of this inhibitory network between the two tasks may constitute a core element of the information-processing bottleneck that produces the limited capacity and resource-like behavior of LPFC activities during dual-task performance.

It could be argued that the present neuronal dual-task effects are solely a manifestation of the task-rule effect ${ }^{33}$, in which, under an identical cue stimulation, a subset of LPFC neurons 
show stronger activity for one stimulus-response rule (e.g., match-rule) than the other (e.g., nonmatch-rule), while a similar number of neurons exhibit an opposite effect. However, in all four of the DMT conditions, memory cue selectivity always tended to decrease relative to SMT as a function of the difficulty of the concurrent attention task. Critically, the reawakening of memory cue selectivity after the conclusion of the attention task demonstrates that the present neuronal effects are strongly associated with the instantaneous processing demands of each of the two concurrent tasks, rather than a trial-by-trial difference in the abstract task-rule or task-context.

In neurophysiology, a widely held view is that, during delayed-response performance, a memorandum of the cue is stored among sustained delay-period activities with cue-specific selectivity in the LPFC $^{24,25,34-37}$, presumably via recurrent processing ${ }^{38}$. However, our results showed that, under dual-task conditions, the spatial selectivity of delay-period activities was remarkably attenuated even in correct trials. This discrepancy raises the question of whether short-term memoranda reside entirely in delay-period activity. On one hand, the observed time-course of delay-period activity in the present dual-task suggests that, under the presence of temporally-overlapping demands for attention task processing, memory cue information was maintained by neural mechanisms other than attenuated LPFC delay-period activities, such as dynamic population coding ${ }^{39,40}$, short-term synaptic plasticity ${ }^{41,42}$, or sustained activity in other brain areas such as the posterior parietal $\operatorname{cortex}^{43,44}$, and that memory cue information was propagated back to LPFC delay-period activities after the conclusion of the attention task. On the other hand, the loss of memory cue information in LPFC delay-period activity was not complete. Even under the presence of the most difficult attention task conditions, delay-period activities still maintained noticeable memory cue information, suggesting that it is premature to conclude that working memory is not maintained by LPFC delay-period activity. Collectively, we interpret the present results as evidence of pluralism in the neural coding scheme for working memory. The sustained firing of LPFC neurons is not necessarily the only basis of 
temporal storage for task content. Alternative neural mechanisms such as population coding or short-term synaptic plasticity may play an important role, when, as exemplified in the present dual-tasks, the processing capacities of LPFC sustained activities are overwhelmed by the need to accommodate temporally-overlapping, multiple lines of task information.

In cognitive psychology, dual-task interference has long been associated with capacity limitation in cognition. Our results suggest that competitive interactions between component task processing within an overlapping neural population can directly explain this effect. While it is still not clear whether or not the present mechanisms apply to other types of dual-task performance, such as those that engage the domain-general central executive functions that are responsible for the coordination of concurrent multimodal processing ${ }^{18,45}$, thus indicating that they serve as a general limiting factor in dual-task performance ${ }^{46}$, the present findings indicate that the neural mechanisms of cognitive capacity limitation can be tractable at the level of single-neuron activities using nonhuman primate models of the dual-task interference effect.

\section{ACKNOWLEDGEMENTS}

The authors thank M. Buckley, J. Duncan, M. Kusunoki and M. Stokes for their comments on the manuscript, and R. Akaishi, K. Mochizuki and A. Tanaka for their helpful discussions. This work was supported by Grant-in-Aids for Scientific Research (Nos. 21240024 and 25240021) from the Ministry of Education, Culture, Sports, Science and Technology (MEXT), Japan, to S.F., and by Research Fellowships for Young Scientists from the Japan Society for the Promotion of Science to K.W.

\section{AUTHOR CONTRIBUTIONS}

K.W. designed the experiment, collected and analyzed the data and wrote the manuscript. S.F. designed the experiment, supervised all aspects of the project and wrote the manuscript. 
COMPETING FINANCIAL INTERESTS: The authors declare no competing financial interests. 


\section{FIGURE LEGENDS}

Figure 1 Behavioral tasks. (a) Event sequence for the attention task in the standard dual-task. Monkeys were required to attend to a target ring and perform a rapid lever-release when its color changed to red. Fixation on FR was required throughout the trial. (b) Location of visual stimuli for the attention task (monkey S). Up and Down rings were presented in the left visual field (contralateral to the recording hemisphere). For monkey A, the configuration was inverted, in that Up and Down rings were placed in the right visual field. (c) Addition of the memory task to the attention task (short trial) in the standard dual-task. After the completion of the attention task, all of the rings disappeared and small place-holders were presented at five possible memory cue locations. (d) Same as in panel (c), but the trial type of the attention task was long trial. (e) Location of memory cue presentation for the memory task (monkey S). Black filled squares indicate the five possible memory cue locations in the standard and easy dual-tasks. Open squares depict the three additional locations used in the single memory task (SMT). For monkey A, the configuration was symmetrically inverted. (f) Combination of the attention and memory tasks in the four DMT and SMT conditions. (g) Location of the recording chamber (outer grey circle) and of the areas of recordings (inner dotted circle) on a lateral view of the monkeys' brain. Data recorded from the frontal eye field (FEF) were excluded from the analyses.

Figure 2 Behavioral performance. (a) Median and distribution of session-by-session percent correct rates in the four attention task conditions in monkeys S (left) and A (right) shown as box plots. Whiskers extend from the box to the lowest (highest) datum point that is still within a 1.5 interquartile range of the lower (upper) quartile. Colored dots indicate values beyond whisker ends. Open black circles indicate mean values. ${ }^{*} P<0.05$, ${ }^{* *} P<0.01,{ }^{* * *} P<0.001$. (b) 
Median and distribution of session-by-session median RTs in the attention task for monkeys S (left) and A (right). In monkey S, lever-release RTs of up to $0.8 \mathrm{~s}$ were allowed in some sessions. (c) Median and distribution of raw session-by-session percent correct rates in the SMT and four DMT conditions for monkeys S (left) and A (right). Dotted line shows mean percent correct rates after a "corrected-for-guessing" transformation (chance-level correction, see Online Methods). Data for SMT come from both $\mathrm{SMT}_{\text {pre }}$ and $\mathrm{SMT}_{\text {post }}$, which gave comparable performances in monkeys S $(P=0.40)$ and A $(P=0.49)$. (d) "Spatial-proximity" hypothesis of dual-task interference. The percent correct rates in DMT-Up and DMT-Down would show a common monotonic trend (right) as a function of spatial proximity between two cues (left). (e) Mean percent correct rates in DMT-Up (solid grey line) and DMT-Down (dashed grey line) as a function of the spatial proximity between two cues. Error bars indicate s.e.m. Results obtained in DMT-FR std, DMT-FR $_{\text {easy }}$, and SMT are shown for reference purpose.

Figure 3 Cue-period activity of a representative neuron in the SMT and four DMT conditions (monkey S, right hemisphere). (a) Cue-period activity in $\mathrm{SMT}_{\text {pre. }}$ A polar plot shows the mean discharge rate of cue-period activity for each cue location. A black bar above each histogram shows the duration of memory cue presentation, and the shaded area shows the analysis time-window of cue-period activity $(0.1-0.5 \mathrm{~s}$ from memory cue onset). $P$-values indicate the result of a Kruskal-Wallis test which compared the cue-period activity in the five memory cue locations that were used in both SMT and DMT. The maximum (minimum) response location was defined as the cue location that elicited the highest (lowest) mean discharge rate in $\mathrm{SMT}_{\text {pre }}$. These locations were selected from among the five cue locations that were used in both SMT and DMT conditions. The histogram bin width is $50 \mathrm{~ms}$. (b-d) Cue-period activity in DMT-Up,

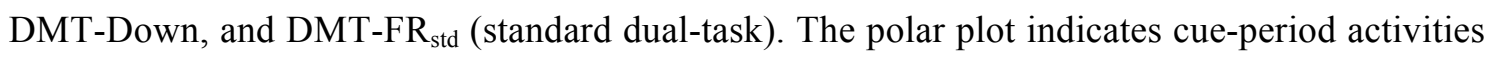
in each DMT condition (blue line) together with that in $\mathrm{SMT}_{\text {pre }}$ (green line). (e) Cue-period

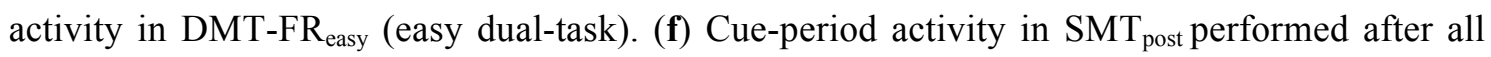


four DMT conditions. The six memory task conditions are labeled alphabetically to match the order of recording, except for $\mathbf{b}-\mathbf{d}$. For this and six other spatially-selective cue neurons in monkey S, the activities in both the standard and easy dual-tasks were recorded.

Figure 4 Delay-period activities of representative neurons. (a) Activity of a single neuron (monkey S, right hemisphere) recorded in the $\mathrm{SMT}_{\text {pre }}$, DMT-Up, DMT-Down, DMT-FR std, and $\mathrm{SMT}_{\text {post }}$ conditions. Raster-histograms for the maximum and minimum response locations are shown in the upper and middle rows, respectively. Shaded area illustrates the analysis time-window for the delay-period activity ( $0-1.0 \mathrm{~s}$ from memory cue offset). In each condition, the second raster-histogram on the right illustrates activities toward the end of trials aligned at the offset of FR (saccade 'go' signal). P-values indicate the result of a Kruskal-Wallis test which compared the delay-period activity in the five memory cue locations that were used in both SMT and DMT. Above each raster-histogram, heavy black and red bars show the duration of memory cue presentation $(0.4 \mathrm{~s})$ and the saccadic response period $(<0.6 \mathrm{~s})$, respectively (memory task events). The dotted heavy black bar shows the duration of attention cue presentation, and the vertical blue and green lines show the mean $\mathrm{T}_{\text {col }}$ change onset timing and the mean lever-release timing, respectively (attention task events). The bottom row shows polar plot diagrams for $\mathrm{SMT}_{\text {pre }}$ (green line) and the remaining four conditions (blue lines). (b) Activity of a single neuron (monkey $\mathrm{S}$, right hemi.) recorded in $\mathrm{SMT}_{\text {pre, }}$ DMT-FR easy, and $\mathrm{SMT}_{\text {post. }}$ In $\mathrm{SMT}_{\text {pre }}$, this neuron exhibited delay-period activity similar to the neuron in panel (a). However, the attenuation of spatial selectivity was absent in DMT-FR easy. .

Figure 5 Population analyses. (a) Population-averaged cue-period activity (grey shaded area) in the maximum (blue line) and minimum (red line) response locations across six memory task conditions. Blue and red shaded areas indicate s.e.m. (b) Population spatial tuning of cue-period activity. The ordinate represents the angular distance relative to the maximum response location 
expressed in radians. On the abscissa, the open circle indicates the mean discharge rate (after baseline subtraction) averaged across all neurons recorded in each memory task condition. The error bar shows the $95 \%$ confidence interval $\left(\mathrm{CI}_{95 \%}\right)$ of the mean calculated by the bootstrap method. Mathematical equation indicates a regression line. For comparison, a regression line obtained in $\mathrm{SMT}_{\text {pre }}$ is shown in the remaining conditions. The regression slope represents the rate of change of the population mean discharge rate above baseline as a function of the angular distance from the maximum response location (one unit $=180 / \pi=57.3^{\circ}$ ), and serves as a measure of population spatial selectivity. $P$-values were derived from a permutation test that examined whether the regression slope $(\beta)$ is significantly different from zero. (c) Population-averaged delay-period activity (grey shaded area). (d) Population spatial tuning of delay-period activity.

Figure 6 Absence of influence of the spatial congruency between a neuron's maximum response location and an attention target ring position on memory task-related activity. (a) Comparison of population cue-period activity in congruent (green) and incongruent (purple) trials. The activity in $\mathrm{SMT}_{\text {pre }}$ (blue) is also shown. Data were obtained from neurons $(\mathrm{n}=40)$ that exhibited spatially-selective cue-period activities in $\mathrm{SMT}_{\text {pre }}$ whose maximum response locations were in the upper visual field $\left(135^{\circ}\right.$ or $90^{\circ}$ in monkey $\mathrm{S} ; 45^{\circ}$ or $90^{\circ}$ in monkey $\mathrm{A}$; Up neuron) or the lower visual field $\left(225^{\circ}\right.$ or $270^{\circ}$ in monkey S; $270^{\circ}$ or $315^{\circ}$ in monkey A; Down neuron). Each neuron's maximum response location was aligned to the upper right visual quadrant. The scatter diagram and frequency histogram contrast the strength of cue-period activity for each neuron between congruent and incongruent trials. Data for Up and Down neurons are separately illustrated by triangles and squares, respectively. For each neuron, the data from two memory cue locations surrounding the attention target ring of congruent trials were collapsed, as depicted by arrows. Dashed lines indicate the mean cue-period activity across 40 neurons. (b) Same as in panel (a), but for delay-period activity $(n=37)$. (c) Comparison of 
the population spatial tuning of cue-period activity between congruent (green) and incongruent trials (purple). Data from the same neurons for $\mathrm{SMT}_{\text {pre }}$ and $\mathrm{SMT}_{\text {post }}$ conditions are also shown for comparison. Conventions as in Fig. 5. (d) Same as in panel (c), but for delay-period activity.

Figure 7 Neuronal responses against attention task events. (a) Population activity at the maximum (cyan) and minimum (orange) response locations aligned at attention cue onset (left panel) and $\mathrm{T}_{\text {col }}$ change onset (right panel) in the standard dual-task. Data were obtained from 61 neurons that exhibited spatially-selective cue-period activity in $\mathrm{SMT}_{\text {pre. }}$ A vertical arrow indicates the earliest possible timing of memory cue presentation (1.6 $\mathrm{s}$ after attention cue offset). Neuronal activity that occurred after the onset of memory cue was excluded from the analysis. A pie chart indicates proportions of three neuron subtypes that were classified according to their response characteristics during the attention cue period. Activities collected during correct trials (regardless of memory cue presentation) were analyzed. (b) Same as in panel (a), but for 51 neurons that exhibited spatially-selective delay-period activity in $\mathrm{SMT}_{\text {pre }}$. Grey shaded area indicates the analysis time window for the attention delay period. (c) Population activity in the $\mathrm{FR}_{\text {easy }}$ condition in the easy dual-task. Data were obtained from 37 neurons with spatially-selective cue-period activity in $\mathrm{SMT}_{\text {pre. }}$ (d) Same as in panel (c), but for 24 neurons with spatially-selective delay-period activity in $\mathrm{SMT}_{\text {pre. }}$ (e) Proportion of neurons that exhibited significant activity modulation relative to the baseline level (Wilcoxon signed-rank test, $P<0.05$ ) during the attention cue period (left) and the attention delay period (right). Data from individual monkeys are shown as open (monkey S) and filled (monkey A) circles. Colors of stacked bar graphs indicate two types of modulation: excitatory (light grey) and inhibitory (dark grey).

Figure 8 Temporal dynamics of neuronal signals representing attention and memory task information in the standard dual-task. (a) Population-averaged time course of neuronal signals 
representing locations of the attention cue ( $\mathrm{PEV}_{\text {attention}}$, magenta), the memory cue ( $\mathrm{PEV}_{\text {memory }}$,

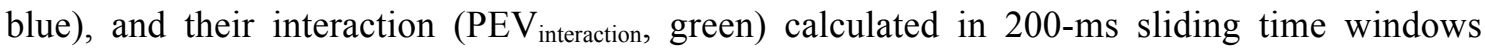
shifted by $20 \mathrm{~ms}$. Data were obtained from neurons that exhibited spatially-selective delay-period activity in $\operatorname{SMT}_{\text {pre }}(n=51)$. From left to right, data are aligned relative to attention cue offset, delay-period onset, $\mathrm{T}_{\text {col }}$ change onset, and lever-release onset. Shaded areas indicate s.e.m. The dashed cyan line indicates same neurons' population-averaged $\mathrm{PEV}_{\text {memory }}$ in $\mathrm{SMT}_{\text {pre }}$. D: Delay-period, P: Pre- $\mathrm{T}_{\text {col }}$ change period, F: Follow-up fixation period, S: Saccade period. (b) Comparison of $\mathrm{PEV}_{\text {memory }}$ between the pre- $\mathrm{T}_{\mathrm{col}}$ change period and the follow-up fixation period in the standard dual-task. The PEV value of each neuron was calculated as the mean of $\mathrm{PEV}_{\text {memory }}$ time series in the corresponding task period. (c) Comparison of $\mathrm{PEV}_{\text {memory }}$ and $\mathrm{PEV}_{\text {attention }}$ during the follow-up fixation period in the standard dual-task. (d) Comparison of $\mathrm{PEV}_{\text {memory }}$ values in the follow-up fixation period between the standard dual-task and $\mathrm{SMT}_{\text {pre }}$. (e) Comparisons of peak $\mathrm{PEV}_{\text {memory }}$ values during the saccade period between the standard

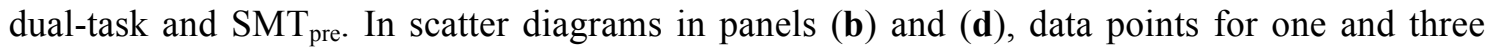
neurons (with $\mathrm{PEV}_{\text {memory }}>0.3$ ), respectively, are not shown. (f) Time courses of the proportion of neurons with significant effects $(P<0.05)$ in each factor. Horizontal dashed lines indicate the proportion expected by chance $(5 \%)$.

\section{REFERENCES}

1. Duncan, J. The demonstration of capacity limitation. Cogn. Psychol. 12, 75-96 (1980).

2. Redelmeier, D.A. \& Tibshirani, R.J. Association between cellular-telephone calls and motor vehicle collisions. N. Engl. J. Med. 336, 453-458 (1997).

3. Strayer, D.L. \& Johnston, W.A. Driven to distraction: dual-task studies of simulated driving and conversing on a cellular telephone. Psychol. Sci. 12, 462-466 (2001).

4. Pashler, H. Dual-task interference in simple tasks: data and theory. Psychol. Bull. 116, 
220-244 (1994).

5. Marois, R. \& Ivanoff, J. Capacity limits of information processing in the brain. Trends Cogn. Sci. 9, 296-305 (2005).

6. Baddeley, A. \& Hitch, G.J. Working memory. in The Psychology of Learning and Motivation, vol. 8 (ed. Bower, G.H.) 47-89 (Academic Press, New York, 1974).

7. Telford, C. W. The refractory phase of voluntary and associative responses. J. Exp. Psychol. 14, 1-36 (1931).

8. Welford, A. The 'psychological refractory period' and the timing of high speed performance - a review and theory. Br. J. Psychol. Gen. Sect. 43, 2-19 (1952).

9. Kahneman, D. Attention and Effort. (Prentice Hall, Englewood Cliffs, New Jersey, 1973).

10. Wickens, C.D. The structure of attentional resources. in Attention and Performance VIII (ed. Nickerson, R.S.) 239-257 (Lawrence Erlbaum, Hillsdale, New Jersey, 1980).

11. Wickens, C.D. Multiple resources and performance prediction. Theor. Issues Ergon. 3, 159-177 (2002).

12. Klingberg, T. Concurrent performance of two working memory tasks: potential mechanisms of interference. Cereb. Cortex 8, 593-601 (1998).

13. Adcock, R.A., Constable, R.T., Gore, J.C. \& Goldman-Rakic, P.S. Functional neuroanatomy of executive processes involved in dual-task performance. Proc. Natl. Acad. Sci. USA 97, 3567-3572 (2000).

14. Bunge, S.A., Klingberg, T., Jacobsen, R.B. \& Gabrieli, J.D.E. A resource model of the neural basis of executive working memory. Proc. Natl. Acad. Sci. USA 97, 3573-3578 (2000).

15. Passingham, R.E. Attention to action. Philos. Trans. R. Soc. Lond., B, Biol. Sci. 351, 1473-1479 (1996).

16. Klingberg, T. \& Roland, P.E. Interference between two concurrent tasks is associated with activation of overlapping fields in the cortex. Brain Res. Cogn. Brain Res. 6, 1-8 (1997). 
17. Baddeley, A., Della Sala, S., Papagno, C. \& Spinnler, H. Dual-task performance in dysexecutive and nondysexecutive patients with a frontal lesion. Neuropsychology 11, 187-194 (1997).

18. D'Esposito, M. et al. The neural basis of the central executive system of working memory. Nature 378, 279-281 (1995).

19. Jiang, Y., Saxe, R. \& Kanwisher, N. Functional magnetic resonance imaging provides new constraints on the theories of the psychological refractory period. Psychol. Sci. 15, 390-396 (2004).

20. Dux, P.E., Ivanoff, J., Asplund, C.L. \& Marois, R. Isolation of a central bottleneck of information processing with time-resolved fMRI. Neuron 52, 1109-1120 (2006).

21. Funahashi, S., Bruce, C.J. \& Goldman-Rakic, P.S. Dorsolateral prefrontal lesions and oculomotor delayed response performance: evidence for mnemonic "scotomas". $J$. Neurosci. 13, 1479-1497 (1993).

22. Rossi, A.F., Bichot, N.P., Desimone, R. \& Ungerleider, L.G. Top down attentional deficits in macaques with lesions of lateral prefrontal cortex. J. Neurosci. 27, 11306-11314 (2007).

23. Zanto, T.P., Rubens, M.T., Thangavel, A. \& Gazzaley, A. Causal role of the prefrontal cortex in top-down modulation of visual processing and working memory. Nat. Neurosci. 14, 656-661 (2011).

24. Funahashi, S., Bruce, C.J. \& Goldman-Rakic, P.S. Mnemonic coding of visual space in the monkey's dorsolateral prefrontal cortex. J. Neurophysiol. 61, 331-349 (1989).

25. Constantinidis, C., Franowicz, M.N. \& Goldman-Rakic, P.S. The sensory nature of mnemonic representation in the primate prefrontal cortex. Nat. Neurosci. 4, 311-316 (2001).

26. Everling, S., Tinsley, C.J., Gaffan, D. \& Duncan, J. Filtering of neural signals by focused attention in the monkey prefrontal cortex. Nat. Neurosci. 5, 671-676 (2002).

27. Lebedev, M.A., Messinger, A., Kralik, J.D. \& Wise, S.P. Representation of attended versus 
remembered locations in prefrontal cortex. PLoS Biol. 2, e365 (2004).

28. Desimone, R. \& Duncan, J. Neural mechanisms of selective visual attention. Annu. Rev. Neurosci. 18, 193-222 (1995).

29. Knudsen, E.I. Fundamental components of attention. Annu. Rev. Neurosci. 30, 57-78 (2007).

30. Fougnie, D. \& Marois, R. Distinct capacity limits for attention and working memory evidence from attentive tracking and visual working memory. Psychol. Sci. 17, 526-534 (2006).

31. Franconeri, S.L., Alvarez, G.A. \& Cavanagh, P. Flexible cognitive resources: competitive content maps for attention and memory. Trends Cogn. Sci. 17, 134-141 (2013).

32. Rao, S.G., Williams, G.V. \& Goldman-Rakic, P.S. Destruction and creation of spatial tuning by disinhibition: GABA(A) blockade of prefrontal cortical neurons engaged by working memory. J. Neurosci. 20, 485-494 (2000).

33. Wallis, J.D., Anderson, K.C., Miller, E.K. Single neurons in prefrontal cortex encode abstract rules. Nature 411, 953-956 (2001).

34. Fuster, J.M. \& Alexander, G.E. Neuron activity related to short-term memory. Science 173, 652-654 (1971).

35. Kubota, K. \& Niki, H. Prefrontal cortical unit activity and delayed alternation performance in monkeys. J. Neurophysiol. 34, 337-347 (1971).

36. Goldman-Rakic, P.S. Cellular basis of working memory. Neuron 14, 477-485 (1995)

37. Curtis, C.E. \& D’Esposito, M. Persistent activity in the prefrontal cortex during working memory. Trends Cogn. Sci. 7, 415-423 (2003).

38. Wang, X.J. Synaptic reverberation underlying mnemonic persistent activity. Trends Neurosci. 24, 455-463 (2001).

39. Meyers, E.M., Freedman, D.J., Kreiman, G., Miller, E.K. \& Poggio, T. Dynamic population coding of category information in inferior temporal and prefrontal cortex. 
J .Neurophysiol. 100, 1407-1419 (2008).

40. Stokes, M. et al. Dynamic coding for cognitive control in prefrontal cortex. Neuron $\mathbf{7 8}$, 364-375 (2013).

41. Zucker, R.S. \& Regehr, W.G. Short-term synaptic plasticity. Annu. Rev. Physiol. 64, 355-405 (2002).

42. Mongillo, G., Barak, O. \& Tsodyks, M. Synaptic theory of working memory. Science $\mathbf{3 1 9}$, 1543-1546 (2008).

43. Katsuki, F. \& Constantinidis, C. Unique and shared roles of the posterior parietal and dorsolateral prefrontal cortex in cognitive functions. Front. Integr. Neurosci. 6:17 (2012).

44. Suzuki, M. \& Gottlieb, J. Distinct neural mechanisms of distractor suppression in the frontal and parietal lobe. Nat. Neurosci. 16, 98-104 (2013).

45. Rissman, J., Gazzaley, A. \& D’Esposito, M. The effect of non-visual working memory load on top-down modulation of visual processing. Neuropsychologia 47, 1637-1646 (2009).

46. Bourke, P.A., Duncan, J. \& Nimmo-Smith, I. A general factor involved in dual-task performance decrement. Q. J. Exp. Psychol. A 49, 525-545 (1996).

\section{REFERENCE in ONLINEMETHODS}

47. Watanabe, K., Igaki, S. \& Funahashi, S. Contribution of prefrontal cue-, delay-, and response-period activity to the decision process of saccade direction in a free-choice ODR task. Neural Netw. 19, 1203-1222 (2006).

48. Robinson, D.A. A method of measuring eye movement using a scleral search coil in a magnetic field. IEEE Trans. Biomed. Eng. 10, 137-145 (1963).

49. Bruce, C.J., Goldberg, M. E., Bushnell, M. C. \& Stanton, G. B. Primate frontal eye fields. II. Physiological anatomical correlates of electrically evoked eye movements. $J$. Neurophysiol. 54, 714-734 (1985). 
50. Page, E.B. Ordered hypotheses for multiple testing: a significance test for linear ranks. $J$. Am. Stat. Assoc. 58, 216-230 (1963).

51. Kutner, M.H., Nachtsheim, C.J., Neter, J. \& Li, W. Applied Linear Statistical Models. (McGraw-Hill, New York, 2005).

52. Buschman, T.J., Siegel, M., Roy, J.E. \& Miller, E.K. Neural substrates of cognitive capacity limitations. Proc. Natl. Acad. Sci. USA 108, 11252-11255 (2011).

53. Kline, R.B. Beyond Significance Testing: Reforming Data Analysis Methods in Behavioral Research. (American Psychological Association, Washington DC, 2004). 


\section{ONLINE METHODS}

\section{Subjects and apparatus.}

Two adult Japanese monkeys (Macaca fuscata; monkey S, male, $9.1 \mathrm{~kg}$; monkey A, female, 5.5 $\mathrm{kg}$ ) were used. Both monkeys were housed individually. The light/dark cycle was 13/11-h (light, from 8:00 a.m. to 9:00 p.m.). Monkey S had temporally participated in an experiment in which single-unit recording was performed in the hemisphere opposite that in the present study. In aseptic surgeries described elsewhere in detail ${ }^{47}$, the recording chamber $(20 \mathrm{~mm}$ diameter, Narishige) was stereotaxically placed on the lateral surface of the prefrontal cortex, under the guidance of structural MRI images. The chamber was placed in the right hemisphere for monkey S, and in the left hemisphere for monkey A. Both monkeys exclusively used the hand ipsilateral to the recording hemisphere for lever manipulation. The monkeys sat in a primate chair in a dark sound-attenuated room with restricted head movements. Visual stimuli were presented on a 21-inch CRT monitor (RD21GZ, Mitsubishi) placed $45 \mathrm{~cm}$ from the monkey's face. The lever (customized microswitch) was attached to the front wall of the chair. Eye movements were monitored using a magnetic scleral search coil system ${ }^{48}$. Eye fixation was controlled within a $6.0^{\circ}$ square window. TEMPO software (Reflective Computing) was used to control behavioral tasks. After the experiment, electrolytic lesions were made at several locations in the recording areas for histological examinations. The monkeys were sacrificed by a standard perfusion protocol. All experimental protocols were approved by the Animal Research Committee at the Graduate School of Human and Environmental Studies, Kyoto University, and were in full compliance with the guidelines of the Primate Research Institute, Kyoto University.

\section{Behavioral paradigm}

The monkeys were first trained in the single attention task, and then in the single memory task (SMT). Training of the two dual-tasks, i.e., the standard and easy dual-tasks, began after the completion of training for both the single attention and memory tasks (above a $90 \%$ correct rate 
in three consecutive sessions). For both dual-tasks, learning of the task was judged to be complete when the monkey achieved an $85 \%$ correct rate in three consecutive sessions. To ensure that the monkeys could readily switch between the blocks of SMT and those of the two dual-tasks, we further continued the training for a month with both SMT and the standard or easy dual-task blocks performed within a single session. The monkeys performed these tasks in the same order as in the recording sessions: $\mathrm{SMT}_{\text {pre }}$ block (typically 150-200 trials), the standard or easy dual-task block (200-300 trials and 80-120 trials, respectively; at least 8 trials were recorded in each memory cue location for each dually-performed memory task condition), and then $\mathrm{SMT}_{\text {post }}$ block (to satiety).

\section{Data collection}

Neural activity was recorded by a glass-coated elgiloy microelectrode (1.0-2.0 M $\Omega$ at $1 \mathrm{kHz})$, advanced by a hydraulic microdrive (MO-95, Narishige). Raw signals were filtered ( $300 \mathrm{~Hz}-10$ $\mathrm{kHz}$ ) and amplified (DAM80, WPI). Single-neuron activity was isolated on-line using a window discriminator (DIS-1, BAK Electronics), and continuously monitored by two oscilloscopes (SS-7802, Iwatsu). Time-stamps of action potentials and behavioral events were stored in magnetic media by TEMPO for analyses. Spike wave forms and raw signals were digitized at $20 \mathrm{kHz}$ (PowerLab 8/35, AD instruments) and stored using custom software (Chart, AD Instruments).

The monkeys performed the SMT condition ( $\mathrm{SMT}_{\text {pre}}$ ) at the beginning of every session during which we searched for well-isolated neuron activities exhibiting task-related activity. After $\mathrm{SMT}_{\text {pre, }}$ the task was switched to either the standard or the easy dual-task, which was randomly selected to approximately match a ratio of $2: 1$. $\mathrm{SMT}_{\text {post }}$ was introduced after the dual-task block. In about $20 \%$ of sessions, the monkeys stopped task performance during the dual-task block. In these cases, we terminated the session without introducing $\mathrm{SMT}_{\text {post. }}$ To exclude neurons that were recorded in the frontal eye field (FEF), intracortical 
microstimulations (22 biphasic pulses, $0.2-\mathrm{ms}$ duration at $333 \mathrm{~Hz}, \leq 150 \mu \mathrm{A}$ ) were applied through microelectrodes. When eye movements were elicited below $50 \mu \mathrm{A}$, the site was considered to be in the low-threshold $\mathrm{FEF}^{49}$, and data obtained at these sites were excluded from the database.

\section{Data analysis}

We analyzed the behavioral and neuronal data in a total of 160 recording sessions ( 74 for monkey S, and 86 for monkey A). All statistical analyses were assessed by two-tailed tests using MATLAB (MathWorks), unless otherwise noted. To avoid an inflated Type I error in multiple hypothesis testing, the significance level was adjusted by Holm's sequentially rejective Bonferroni (SRB) procedure, or by appropriate post-hoc tests following omnibus statistical tests. No statistical tests were run to determine sample sizes (the number of neurons that exhibited task-related activities) a priori. The sample sizes we chose approximately match those used in previous publications. Although no blinding was done regarding the assignment of individual neuron samples to the two experimental groups (standard and easy dual-tasks), the results in Supplementary Fig. 8 indicate that the response properties of neurons in the control condition (SMT) were highly similar between the two groups, demonstrating that the present group allocation was unbiased.

\section{Analysis of attention task performance}

To determine whether the task difficulty in the four attention task conditions differed, we compared the percent correct rates and lever-release response times in dual-task trials. Since we were interested in all pairwise comparisons among the four attention task conditions, we performed a series of six pairwise comparisons using either the Wilcoxon signed-rank test (for three comparisons involving paired data among three intermingled attention conditions in the standard dual-task, i.e., Up, Down and $\mathrm{FR}_{\text {std }}$ conditions) or the Wilcoxon rank-sum test, with 
Holm's SRB procedure. The significance level for this procedure started at $0.05 / 6$. There were three types of errors in the attention task: (1) a fixation break (FB) error before $\mathrm{T}_{\text {col }}$ change, (2) premature lever-release before $\mathrm{T}_{\mathrm{col}}$ change, and (3) failure to initiate lever-release within $0.6 \mathrm{~s}$ after $T_{\text {col }}$ change. Only the latter two types of errors were considered in calculating the percent correct rates. Separate analyses showed that FB errors were rare and mostly attributable to the onset of the memory cue (Supplementary Fig. 2b-d), which validated the exclusion of FB errors from this analysis.

\section{Analysis of memory task performance.}

To determine the presence or absence of dual-task interference in memory task performance, percent correct rates were compared across the SMT and four DMT conditions. We performed a series of 10 pairwise comparisons using either the Wilcoxon signed-rank test (for three comparisons involving paired data among three intermingled DMT conditions in the standard dual-task) or the Wilcoxon rank-sum test (remaining seven comparisons) with Holm's SRB procedure (the initial significance level was set at 0.05/10). To obtain session-by-session percent correct rates, the number of correct trials was divided by the number of trials in which monkeys successfully completed the attention task component (i.e., trials in which lever-release was made within $0.6 \mathrm{~s}$ of $\mathrm{T}_{\text {col }}$ change onset). Here, correct trials in the memory task were defined as those that resulted in both (1) successful saccadic target-acquisition within $0.6 \mathrm{~s}$ after place-holder onset, and (2) successful gaze-keeping at a correct place-holder for $0.6 \mathrm{~s}$. All other types of eye movements that occurred after lever-release in the attention task were considered to be errors. Since the SMT condition did not involve $\mathrm{T}_{\mathrm{col}}$ change or a subsequent lever-release, both events were scheduled but executed as 'empty events' without any change in the physical stimuli or a behavioral response.

For a fair comparison of memory task performance between the SMT and DMT conditions, we equalized the time window for calculating percent correct rates. In SMT, relative 
to place-holder onset (saccade 'go' signal), the timing of the 'empty' $\mathrm{T}_{\mathrm{col}}$ change onset was set at $-0.9 \mathrm{~s}$, and the timing of the 'empty' lever-release was set at $-0.4 \mathrm{~s}$. This $0.4-\mathrm{s}$ time window between the 'empty' lever-release and place-holder onset was considered to be a pseudo follow-up fixation period that corresponded to the actual follow-up fixation period in DMT. FB errors that occurred during these pseudo and actual follow-up fixation periods in the SMT and DMT conditions, respectively, were incorporated into the calculation of the percent correct rate. Note that during performance of the standard and easy dual-tasks, a follow-up fixation period was also required in single attention task trials in which the memory cue was not presented. This was to ensure that lever-release per se did not disrupt the oculomotor control required in memory-guided saccades in dual-task trials. We confirmed that FB errors rarely occurred after lever-release in these single attention task trials; median FB error rates were $0 \%$ in all four attention task conditions, indicating that lever-release per se did not disrupt oculomotor control. The additional analysis that excluded trials that ended with (1) FB errors in the follow-up fixation period and (2) gaze-keeping errors after the saccade gave results that were qualitatively identical to those reported in the main text. The percent correct rate for SMT was calculated by excluding the three (out of eight) memory cue locations that were not used in DMT (Fig. 1e).

To compensate for a chance-level difference between SMT (12.5\%) and DMT (20\%), we also examined memory task performance after transforming the raw percent correct rates of each session into "corrected-for-guessing" scores. This transformation was made by using a conventional formula, $\mathrm{S}=[\mathrm{C}-\mathrm{E} /(n-1)] /(\mathrm{C}+\mathrm{E})$, where $\mathrm{S}$ is the corrected score, $\mathrm{C}$ is the number of correct responses, $\mathrm{E}$ is the number of error responses, and $n$ is the number of alternatives (i.e., eight in SMT; five in DMT). The results obtained by this procedure were qualitatively identical to the raw percent correct rates, as shown in Fig. 2c.

In the DMT-Up and DMT-Down conditions, since the attention and memory cues could be located close to or far from each other, it is possible that memory task performance under these conditions could be accounted for by the spatial proximity of the two cues 
("spatial-proximity" hypothesis of dual-task interference, Fig. 2d). Under this hypothesis, the percent correct rates in DMT-Up and DMT-Down would show a common monotonic trend as a function of spatial proximity between the attention and memory cues. The presence of a significant monotonic trend was assessed by a non-parametric trend test for repeated-measures data (Page's $L$ test) ${ }^{50}$. A test static, $L$, represents the degree of association between the ordered conditions (spatial proximity) and the observed score (percent correct rate), which is calculated as the sum of the product of the predicted group rank (ordered between 1 and the number of levels in the condition) and the observed rank of each group score within each session (from 1 to the number of levels in the condition), with average ranks given to tied scores. To infer statistical significance, the computed $L$ value was compared with the null distribution estimated from 10,000 within-group permutations of randomly shuffled spatial proximity levels.

\section{Analysis of spatial selectivity in single-neuron activity}

To investigate the neural mechanisms of dual-task interference, we compared the strength of spatial selectivity for memory cue location across the SMT and DMT conditions. Neurons that exhibited significant spatial selectivity (Kruskal-Wallis test, $P<0.05$ ) among eight memory cue locations that covered both visual hemifields were included in the analysis database. All subsequent comparisons between SMT and DMT were made using five cue locations that were used in both the SMT and DMT conditions. For each neuron that exhibited spatial selectivity in $\mathrm{SMT}_{\text {pre }}$, the maximum (minimum) response location was defined as the cue location that elicited the highest (lowest) mean discharge rate in $\mathrm{SMT}_{\text {pre }}$. These locations were selected from among the five cue locations.

\section{Computation of a population tuning plot}

To construct a population spatial tuning plot, we first obtained a tuning plot for each neuron that yielded three to five data points depending on the maximum response location. To calculate 
each neuron's tuning plot, the activities in the five memory cue locations presented during both SMT and DMT were used. For each of the five memory cue locations, the baseline discharge rate (mean discharge rate during a 0.8 -s time window before memory cue onset, pooled across five memory cue locations) was subtracted from the mean discharge rate during the period of interest. These five values were assigned to one of five location categories, depending on the angular distance from the maximum response location of the neuron $\left(0^{\circ}, 45^{\circ}, 90^{\circ}, 135^{\circ}\right.$, and $180^{\circ}$ apart). If two different discharge rates were assigned to one location category, the mean of these two values was used. For example, a neuron recorded from monkey $\mathrm{S}$ with spatially-selective cue-period activity with a maximum response location at $135^{\circ}$ gives four data points $\left(\mathrm{x}^{\circ}\right.$ apart, $\mathrm{y}$ spikes per $\left.\mathrm{s}\right):\left(0^{\circ}\right.$ apart, mean discharge rate at $\left.135^{\circ}\right),\left(45^{\circ}\right.$ apart, mean of mean discharge rates at $90^{\circ}$ and $\left.180^{\circ}\right),\left(90^{\circ}\right.$ apart, mean discharge rate at $\left.225^{\circ}\right)$, and $\left(135^{\circ}\right.$ apart, mean discharge rate at $270^{\circ}$ ). Data points obtained at each location category were accumulated across all spatially-selective neurons, and an ordinary least squares (OLS) regression analysis was performed.

To compensate for the presence of significant outliers that could distort the accuracy of regression, we additionally performed two standard remedial variants of OLS regression ${ }^{51}$ : (1) OLS regression with outlier deletion by Cook's distance measure, and (2) iteratively reweighted least squares (IRLS) robust regression with Tukey's bisquare weight function. We confirmed that both analyses yielded results that were qualitatively identical to those of OLS regression. Since OLS regression with Cook's distance measure gave the highest $R$-squared measure of goodness of fit, and only a small fraction of data points (mean, 4.2\%) were considered to be outliers, we adopted the results obtained by this method. Cook's distance measure, denoted by $D_{i}$, is an aggregate influence measure, which shows the effects of the $i$ th data point on all $n$ fitted values:

$$
D_{i}=\frac{\sum_{j=1}^{n}\left(\widehat{Y}_{j}-\hat{Y}_{j(i)}\right)^{2}}{p M S E}
$$


where $\hat{Y}_{j}$ is the prediction from the full regression model for observation $j ; \hat{Y}_{j(i)}$ is the prediction for observation $j$ from a refitted regression model in which observation $i$ has been omitted; MSE is the mean square error of the regression model; and $p$ is the number of fitted parameters in the regression model. According to the standard criterion, we treated data point $i$ as an outlier if $D_{i}>4 /(n-k-1)$, where $n$ is the number of data points and $k$ the number of explanatory variables $(k=1)$. We examined whether the slope of the regression line $(\beta)$ was significantly different from zero $\left(\mathrm{H}_{0}, \beta=0 ; \mathrm{H}_{1}, \beta \neq 0\right)$ by the permutation test. The observed slope was compared with the null distribution of the slope values which was obtained by re-estimation of the slope in 100,000 permutations, while allowing permutation $P$-values as small as $10^{-5}$.

\section{Computation of a proportion of explained variance}

We quantified the strength of selectivity for memory cue location carried by the activity of each neuron using a proportion of explained variance (PEV), which was measured by the $\omega^{2}$ index of the effect size. The $\omega^{2} \mathrm{PEV}$ indicates how much of the variance in the trial-by-trial firing rate of a neuron can be explained by the location of memory cue presentation, and makes no assumption about the consistency of selectivity over time or condition. We selected the $\omega^{2}$ index for the present analysis, since it has been commonly used in the previous studies to quantify the strength of neuronal selectivity (e.g., ref 52), and can be extended to the analysis of data in a multi-factorial design using a partial $\omega^{2}$ index. The $\omega^{2}$ has the advantage of being a more accurate estimator of the population effect size than the $\eta^{2}$ index with small sample sizes ${ }^{53}$.

The $\omega^{2}$ PEV values in Supplementary Fig. 9 (one-way design with a factor Memory cue location) were calculated by the formula:

$$
\omega^{2}=\frac{S S_{\text {effect }}-d f_{\text {effect }} \times M S E}{S S_{\text {total }}+M S E}
$$

where $S S_{\text {effect }}$ is the sum of squares between groups, $d f_{\text {effect }}$ is the degree of freedom for the factor 
for which the effect size is being estimated (Memory cue location), $S S_{\text {total }}$ is the total sum of squares for the data set, and MSE is the mean squared error within groups. Negative values may result when $F<1$.0. For the analyses in Fig. 8 and Supplementary Fig. 11, we used a partial $\omega^{2}$ proportion of explained variance (PEV) to quantitatively analyze the time course of the strength of information about attention cue location (3 levels, $\mathrm{PEV}_{\text {attention }}$ ), memory cue location (5 levels, $\left.\mathrm{PEV}_{\text {memory }}\right)$, and their interaction $\left(\mathrm{PEV}_{\text {interaction }}\right)$ carried by each LPFC neuron in the standard dual-task. For $\mathrm{SMT}_{\text {pre }}$ and the easy dual-task (DMT-FR $\mathrm{easy}_{\mathrm{f}}$ ), only the time course of $\mathrm{PEV}_{\text {memory }}$ was calculated using the partial $\omega^{2}$ PEV. Note that in one-way designs (the easy dual-task and SMT), the $\omega^{2}$ and partial $\omega^{2}$ PEV values are identical for omnibus comparisons. In the present completely between-subjects two-way design (standard dual-task), a partial $\omega^{2} \mathrm{PEV}$ in each 200-ms sliding time window was calculated by the formula:

$$
\omega_{p}^{2}=\frac{d f_{\text {effect }}\left(F_{\text {effect }}-1\right)}{d f_{\text {effect }}\left(F_{\text {effect }}-1\right) \times N}
$$

where $d f_{\text {effect }}$ and $F_{\text {effect }}$ are the degree of freedom and the $F$ value of the factor under consideration, respectively, and $N$ is the total number of samples. The partial $\omega^{2}$ eliminates the influence of other factors in the design, and thus is resistant to the difference in the number of factors when comparing the resultant values from different comparisons ${ }^{53}$. To confirm the compatibility of the comparison between the partial $\omega^{2} \mathrm{PEV}_{\text {memory }}$ in the standard dual-task (two-way design) and that in $\mathrm{SMT}_{\text {pre }}$ (one-way design), we recalculated the time course of $\mathrm{PEV}_{\text {memory }}$ in $\mathrm{SMT}_{\text {pre }}$ in a pseudo two-way design, in which a dummy factor, Attention cue location, was introduced by randomly assigning each SMT trial to either the Up, Down, or FR std attention task condition. The resultant time course of $\mathrm{PEV}_{\text {memory }}$ was almost identical to that reported in the main text, and the average time-course of $\mathrm{PEV}_{\text {memory }}$ derived from multiple recalculations converged to the values reported in the main text. 


\section{SUPPLEMENTARY FIGURE LEGENDS}

Supplementary Figure 1 Event sequence of example trials. (a) Example trials in the standard dual-task. The upper row depicts an example dual-task trial in which the attention task is performed as a short trial. The bottom row shows a trial in which the attention task is performed as a long trial. Note that, in the long trial, there were two possible patterns in the temporal order of catch change and memory cue presentation. (b) Same as in panel (a), but for the easy dual-task. Note that, in the long trial (bottom row), catch change was scheduled and executed as an 'empty event'. (c) Example trials in the single memory task (SMT). The time course of the task was matched with that in the standard and easy dual-tasks. However, while all attention task events were scheduled, they were executed as 'empty events'. Trials were automatically initiated by the appearance of FR after an intertrial interval (4.0-7.0 s).

Supplementary Figure 2 Additional evidence supporting the presence of dual-task interference effect in the DMT conditions. (a) Mean percent correct rates in the SMT and four DMT conditions plotted separately for the trials with short $(<2.0 \mathrm{~s})$, medium $(2.0-4.0 \mathrm{~s})$ and long (> $4.0 \mathrm{~s})$ memory delay periods. In monkey S (left), a two-way mixed-design ANOVA showed significant main effects of both Task condition and Delay length $\left(P<10^{-4}\right)$, and a nonsignificant interaction effect $(P=0.27)$. In monkey A (right), there were significant main effects of both Task condition and Delay length $\left(P<10^{-4}\right)$, and an interaction effect $(P=0.02)$. Asterisks indicate the result of the simple effect ANOVA for the factor Delay length. (b) Time course of FB error rates relative to memory cue onset averaged across all sessions for monkeys S (left) and A (right). Inset bar graphs indicate the mean FB error rate during the 1-s period following memory cue onset. Error bars indicate s.e.m. During this period, monkey S showed a significant increase in the FB error rate in DMT-Up and DMT-Down compared with SMT 
(post-hoc Steel-Dwass test, $P<10^{-4}$; omnibus Kruskal-Wallis test, $P<10^{-4}$ ), indicating that the oculomotor aspect of memory task performance was substantially interfered with by the concurrent attention task. (c, d) Trajectories and end points of FB eye movements that occurred during the 1-s period following memory cue onset in monkeys S (c) and A (d). Three unfilled black circles indicate the ring stimuli for the attention task. Colors are assigned to each memory cue location (square) and each FB eye movement trajectory so that the color of a given FB eye movement trajectory indicates the location of the memory cue presentation that preceded this FB error. End points of FB eye movements are shown as black dots. Numbers shown on each colored square indicate the cumulative number of FB errors across sessions in each memory cue location. The total number of trials $(\mathrm{N})$ in which the memory cue was presented is shown at the bottom of each panel. In the DMT-Up and DMT-Down conditions in monkey S, regardless of memory cue location, FB eye movements after memory cue onset were predominantly directed toward the attention target ring, rather than the memory cue location as observed in SMT. However, importantly, at the time of memory cue onset, the attention cue had been removed from the monitor for 1.6-5.1 s, and the monkeys were simply viewing the still images of three rings. This indicates that around the time of memory cue presentation, information regarding the position of the target ring for the attention task was occupying monkey S's processing capacity, suggesting that the level of readiness for memory cue encoding was severely disrupted. Monkey A's FB errors were characterized by short eye movements clustered on the vertical axis, regardless of the memory task conditions. The similarity in FB eye movement trajectories between the SMT and DMT conditions indicates that in all of the four DMT conditions, the preparatory state for memory cue encoding was not disrupted by the concurrent attention task. Thus, we concluded that, for monkey A, dual-task interference on the oculomotor aspect memory task performance was minimal.

Supplementary Figure 3 Behavioral performance in the modified standard dual-task. (a) 
Schematic diagram of the event sequence for non-cued trials of the attention task that were randomly inserted among the Up, Down, $\mathrm{FR}_{\text {std }}$ conditions (cued trials). A catch change was scheduled but executed as an 'empty event' without actual changes in the display items. (b) Distribution of the session-by-session percent correct rates in the attention task in the modified standard dual-task (consecutive 25 sessions in each monkey) in monkeys S (left) and A (right). Only data from cued trials are shown. The results in $\mathrm{FR}_{\text {easy }}$ (Fig. 2a) are also shown in the rightmost box plot. The statistical testing procedure and conventions were the same as in Fig. 2a. The results in FR easy $($ Fig. 2b) are also shown in the rightmost box plot. (c) Distribution of the session-by-session median RTs in the attention task for monkeys S (left) and A (right).

Distribution of the session-by-session percent correct rates in the three DMT conditions for monkeys S (left) and A (right). The results in SMT (Fig. 2c) are also shown in the rightmost box plot. The dotted line indicates the mean percent correct rates after "corrected-for-guessing" transformation. $P$-values were adjusted for three multiple comparisons between the SMT and three DMT conditions. (e) Comparison of the session-by-session percent correct rates between cued $(\mathrm{C})$ and non-cued $(\mathrm{NC})$ trials in the attention task. In monkey S, the percent correct rates in cued trials were significantly higher than those in non-cued trials (two-way repeated-measures ANOVA: main effect of Cueing, $P=2 \times 10^{-4}$; Attention condition, $P<10^{-4}$; interaction, $P<$ $10^{-4}$ ) (simple main effect of Cueing: Up, $P<10^{-4}$; $\mathrm{FR}_{\text {std }}, P=0.006$; both $\mathrm{C}>\mathrm{NC}$ ). (f) Comparison of the session-by-session median lever-release RTs between cued (C) and non-cued (NC) trials in the attention task. In both monkeys, the RTs in cued trials were significantly shorter than those in non-cued trials (monkey S: main effect of Cueing, $P=5 \times 10^{-4}$; Attention condition, $P<10^{-4}$; interaction, $P=0.06$; monkey A: main effect of Cueing, $P<10^{-4}$; Attention condition, $P=0.17$; interaction, $P=0.09$ ) (simple main effect of Cueing: $P<0.009$ for the Up and Down conditions in monkey S; $P<2 \times 10^{-4}$ for all three conditions in monkey A; all NC $>$ C). 
Supplementary Figure 4 Cue-period activity of example neurons. (a) Activity of a single neuron (monkey S, right hemi.) recorded in the $\mathrm{SMT}_{\text {pre }}$, DMT-Up, DMT-Down, DMT-FR std (standard dual-task), and $\mathrm{SMT}_{\text {post }}$ conditions. Conventions as in Fig. 4. (b) Same as in panel (a), but for a neuron recorded from monkey A (left hemi.) This neuron exhibited significant spatial selectivity in all three DMT conditions in the standard dual-task. However, the strength of cue-period activity in the maximum response location $\left(270^{\circ}\right)$ is significantly attenuated in DMT-Up and DMT-Down compared to that in $\mathrm{SMT}_{\text {pre. }}$ (c) Activity of a single neuron (monkey $\mathrm{A}$, left hemi.) recorded in the $\mathrm{SMT}_{\text {pre, }}$ DMT-FR easy (easy dual-task), and $\mathrm{SMT}_{\text {post }}$ conditions. In DMT-FR $_{\text {easy, }}$ attenuation of both the magnitude and selectivity of cue-period activity was absent.

Supplementary Figure 5 Delay-period activity of example neurons. (a) Activity of a single neuron (monkey S, right hemi.) recorded in the $\mathrm{SMT}_{\text {pre, }}$ DMT-Up, DMT-Down, DMT-FR $\mathrm{std}_{\text {, }}$ DMT-FR easy, and $\mathrm{SMT}_{\text {post }}$ conditions. Conventions as in Fig. 4. From left to right, seven memory task conditions, including two $\mathrm{SMT}_{\text {post }}$ blocks $\left(\mathrm{SMT}_{\text {post }}{ }^{-1}\right.$ and $\left.\mathrm{SMT}_{\text {post }}-2\right)$, are shown in the order

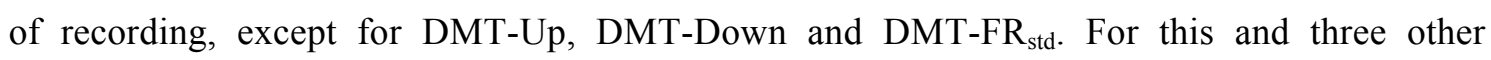
neurons (monkey S) with spatially-selective delay-period activity in $\mathrm{SMT}_{\text {pre, }}$ activities were obtained in both the standard and easy dual-tasks. (b) Activity of a single neuron (monkey A, left hemi.) recorded in $\mathrm{SMT}_{\text {pre, }}$ DMT-Up, DMT-Down, DMT-FR std, and $\mathrm{SMT}_{\text {post. }}$ Note that in DMT-Down, the activity level was elevated in all memory cue locations because the attention cue had been presented near the neuron's maximum response location $\left(315^{\circ}\right)$. Nevertheless, spatial selectivity of delay-period activity was lost in this condition $(P=0.75)$. (c) Activity of a single neuron (monkey A, left hemi.) recorded in $\mathrm{SMT}_{\text {pre, }} \mathrm{DMT}-\mathrm{FR}_{\text {easy }}$, and $\mathrm{SMT}_{\text {post. }}$ This neuron exhibited delay-period activity similar to that in panel (b). However, attenuation of both the magnitude and selectivity of delay-period activity was absent in DMT-FR easy. $_{\text {. }}$ 
Supplementary Figure 6 Population cue-period activities of individual monkeys. (a, b) Data are for monkeys S (a) and A (b). Upper row: population cue-period activity in the maximum (blue line) and minimum (red line) response locations across the six memory task conditions. In both monkeys, significant interaction effects [Task condition $\times$ Cue location] were observed among cue-period activity (monkey S: main effect of Task condition, $F_{5,187}=10.85, P<10^{-4}$; Cue location, $F_{1,187}=114.88, P<10^{-4}$; interaction, $F_{5,187}=10.52, P<10^{-4}$; monkey A: main effect of Task condition, $F_{5,185}=0.54, P=0.76$; Cue location, $F_{1,185}=154.90, P<10^{-4}$; interaction, $F_{5,185}=3.57, P=0.004$; two-way mixed design ANOVA). Bottom row: population spatial tuning during the cue-period. Conventions as in Fig. 5. Although, in both monkeys, cue-period activity exhibited a significant attenuation of spatial selectivity under DMT, the degree of attenuation was smaller in monkey A, whose cue-period activity exhibited a robust increase following memory cue onset. This suggests that, in monkey A, information processing of the memory task was rather unaffected in the initial phase (time period immediately following memory cue presentation), whereas in monkey S, processing of the memory task was considerably disrupted from this phase. In agreement with this notion, comparison of the behavioral performance between the two monkeys showed that monkey A exhibited a more moderate dual-task interference on memory task performance than monkey S (Fig. 2c). This was particularly evident in the trials that had short memory delay period $\left(<\begin{array}{lll}2.0 & \mathrm{~s}\end{array}\right)$ (Supplementary Fig. 2a). In addition, monkey A's oculomotor behavior following memory cue presentation did not show signs of dual-task interference, whereas monkey S's oculomotor behavior in the same epoch clearly exhibited interference by the attention task, as indicated by a significant increase in FB error rates in DMT-Up and DMT-Down relative to SMT (Supplementary Fig. 2b-d).

Supplementary Figure 7 Population delay-period activities of individual monkeys. (a, b) Data are for monkeys S (a) and A (b). Upper row: population delay-period activity in the 
maximum (blue line) and minimum (red line) response locations across the six memory task conditions. In both monkeys, significant interaction effects [Task condition $\times$ Cue location] were observed among delay-period activity (monkey S: main effect of Task condition, $F_{5,126}=$ 4.10, $P=0.002$; Cue location, $F_{1,126}=66.05, P<10^{-4}$; interaction, $F_{5,126}=5.13, P=3 \times 10^{-4}$; monkey A: main effect of Task condition, $F_{5,165}=0.64, P=0.67$; Cue location, $F_{1,165}=65.64, P$ $<10^{-4}$; interaction, $F_{5,165}=3.77, P=0.003$ ). Bottom row: population spatial tuning during the delay-period. Conventions as in Fig. 5. In contrast to the cue-period activity, the degree of selectivity attenuation among delay-period activity was comparable between the two monkeys in the DMT conditions, suggesting that, in both monkeys, memory task processing was substantially disrupted in the later stage by the presence of the concurrent attention task. In accordance with this notion, behavioral results showed that, in both monkeys, prominent dual-task interference was observed in the trials that had long memory delay-period ( $>4.0 \mathrm{~s})$ (Supplementary Fig. 2a). The close correspondence between the individual variability among behavioral performance and that among response patterns of cue- and delay-period activities further supports the notion that the attenuation of neuronal selectivity for the memory cue location under DMT is a direct neural correlate of the behavioral cost of dual-task performance.

Supplementary Figure 8 Comparison of cue- and delay-period activities in SMT between neurons assigned to the recording in the standard dual-task and the easy dual-task. (a,b) Upper row: population cue-period activities in the $\mathrm{SMT}_{\text {pre }}$ (a) and $\mathrm{SMT}_{\text {post }}(\mathbf{b})$ conditions for neurons assigned to the recording in the standard dual-task (left) and the easy dual-task (right). Conventions are the same as in Fig. 5. In both conditions, the activity patterns were highly similar between the groups of neurons assigned to the standard dual-task (left) and the easy dual-task (right) ( $\mathrm{SMT}_{\text {pre: }}$ main effect of Task assignment, $F_{1,96}=0.83, P=0.36$; Cue location, $F_{1,96}=170.30, P<10^{-4}$; interaction, $F_{1,96}=0.06, P=0.81 ; \mathrm{SMT}_{\text {post }}$ : main effect of Task assignment, $F_{1,77}=0.40, P=0.53$; Cue location, $F_{1,77}=67.03, P<10^{-4}$; interaction, $F_{1,77}=0.07$, 
$P=0.79$; two-way mixed-design ANOVA). Bottom row: population spatial tuning during the cue-period in $\mathrm{SMT}_{\text {pre }}(\mathbf{a})$ and $\mathrm{SMT}_{\text {post }}(\mathbf{b})$. In both $\mathrm{SMT}_{\text {pre }}$ and $\mathrm{SMT}_{\text {post }}$, the tuning slopes and intercepts did not differ between the assigned task ( $\mathrm{SMT}_{\text {pre}}$ : slope, $P=0.92$; intercept, $P=0.44$; SMT $_{\text {post: }}$ slope, $P=0.56$; intercept, $\left.P=0.60\right)$. (c,d) Same as in (a) and (b), but for delay-period activity $\left(\mathrm{SMT}_{\text {pre }}\right.$ : main effect of Task assignment, $F_{1,73}=0.16, P=0.69$; Cue location, $F_{1,73}=$ 92.18, $P<10^{-4}$; interaction, $F_{1,73}=0.28, P=0.60$; $\mathrm{SMT}_{\text {post: }}$ main effect of Task assignment, $F_{1,56}=0.78, P=0.38$; Cue location, $F_{1,56}=43.21, P<10^{-4}$; interaction, $\left.F_{1,56}=1.54, P=0.22\right)$. The tuning slopes and intercepts did not differ between the assigned task $\left(\mathrm{SMT}_{\text {pre: }}\right.$ slope, $P=$ 0.65 ; intercept, $P=0.26$; $\mathrm{SMT}_{\text {post }}$ : slope, $P=0.49$; intercept, $P=0.10$ ).

Supplementary Figure 9 Comparison of single-neuron PEV values between the SMT and DMT conditions. (a) Upper row: scatter diagrams comparing PEV values of cue-period activity in $\mathrm{SMT}_{\text {pre }}$ to those in the four DMT and $\mathrm{SMT}_{\text {post }}$ conditions. Integration time window for PEV calculation was $0.4 \mathrm{~s}(0.1-0.5 \mathrm{~s}$ from memory cue onset). Blue dashed lines indicate the mean PEV values in $\mathrm{SMT}_{\text {pre. }}$ Red dashed lines indicate the same neurons' mean PEV values in the corresponding conditions for comparison. Fractions show the number of neurons that showed a decrease in PEV relative to $\mathrm{SMT}_{\text {pre, }}$ divided by the number of neurons that exhibited spatially-selective cue-period activity in $\mathrm{SMT}_{\text {pre }}$ Bottom row: histograms comparing the distribution of PEV values between $\mathrm{SMT}_{\text {pre }}$ (blue bars) vs. each of the four DMT and $\mathrm{SMT}_{\text {post }}$ conditions (inverted red bars). (b) Same as in (a), but for delay-period activity. Integration time window was $1.0 \mathrm{~s}(0-1.0 \mathrm{~s}$ from memory cue offset). (c) Summary of the five paired-comparisons shown in (a). Note that $n=91$ for $\mathrm{SMT}_{\text {pre. The center of a notched bar }}$ indicates the median value, edges are $\mathrm{CI}_{68 \%}$, and the error bar is the $\mathrm{CI}_{95 \%}$ of the median (bootstrap method). Open black circles indicate mean values. PEV values for cue-period activity were significantly different across memory task conditions (Kruskal-Wallis test, $P=4 \times 10^{-4}$ ), and $\mathrm{SMT}_{\text {pre }}$ showed a significantly greater PEV value than DMT-Up, DMT-Down, and 
DMT-FR $_{\text {std }}$ (post-hoc Steel-Dwass test, $P<0.02$ ). All six memory task conditions gave median PEV values significantly larger than zero (one-sample Wilcoxon signed-rank test). (d) Summary of the five paired-comparisons in (b). Note that $n=71$ for $\mathrm{SMT}_{\text {pre. }}$ PEV values in delay-period activity were significantly different across memory task conditions (Kruskal-Wallis test, $P=0.001$ ). $\mathrm{SMT}_{\text {pre }}$ showed a significantly greater $\mathrm{PEV}$ value than DMT-Up, DMT-Down (post-hoc Steel-Dwass test, $P<0.03$ ) and a substantially greater PEV value than DMT-FR $\mathrm{Dtd}_{\text {st }}(P=0.06)$. All six memory task conditions gave median PEV values significantly larger than zero.

Supplementary Figure 10 Comparison of memory task-related activity between the 3-ring and 1-ring layout types in the modified single memory task. (a) Spatially-selective cue-period activity of a representative neuron that exhibited almost identical activities in the two layout types. Conventions as in Fig. 3. (b) Population activity in the 3-ring (top left) and 1-ring layout types (top right) for 13 spatially-selective cue neurons. A scatter diagram (bottom) shows a comparison of the strength of cue-period activity in the maximum (blue) and minimum (red) response locations that were selected from the five cue locations that were also used in DMT. Dotted lines indicate the mean cue-period activity across the population. The strength of cue-period activity was comparable between the two ring layout types at both the maximum $(P$ $=0.31)$ and minimum $(P=0.19)$ response locations (Wilcoxon signed-rank test). (c) Same as in panel (b), but for 10 spatially-selective delay neurons. There was no significant difference in the strength of delay-period activity between the two layout types at both the maximum $(P=0.92)$ and minimum $(P=0.43)$ response locations.

Supplementary Figure 11 Temporal dynamics of neuronal signals representing attention and memory task information in the standard and easy dual-tasks. (a) Time course of neuronal signals of an example neuron (the neuron shown in Supplementary Fig. 5b) representing the 
location of the attention cue ( $\mathrm{PEV}_{\text {attention }}$, magenta), the memory cue ( $\mathrm{PEV}_{\text {memory }}$, blue), and their interaction $\left(\mathrm{PEV}_{\text {interaction}}\right.$, green) in the standard dual-task. Dashed cyan line indicates the same neuron's $\mathrm{PEV}_{\text {memory }}$ in $\mathrm{SMT}_{\text {pre. }}$ Conventions are the same as in Fig. 8a. (b) Time course of neuronal signals of an example neuron (the neuron shown in Supplementary Fig. 5c) representing the location of the memory cue $\left(\mathrm{PEV}_{\text {memory }}\right.$, blue) in the easy dual-task. Dashed cyan line indicates the same neuron's $\mathrm{PEV}_{\text {memory }}$ obtained in $\mathrm{SMT}_{\text {pre. }}$ (c) Population-averaged time course of $\mathrm{PEV}_{\text {memory }}$ in the easy dual-task (solid blue line, $n=24$ ). Shaded areas indicate s.e.m. The same neurons' population-averaged $\mathrm{PEV}_{\text {memory }}$ time series in $\mathrm{SMT}_{\text {pre }}$ are plotted as a solid cyan line. Dashed blue line and dashed cyan line indicate population-averaged $\mathrm{PEV}_{\text {memory }}$ time series in the standard dual-task and $\mathrm{SMT}_{\text {pre }}$, respectively for 51 neurons analyzed in Fig. 8 (the curves are the same as those shown in Fig. 8a). (d) Time course of the proportion of neurons that exhibited significant information $(P<0.05)$ about the memory cue location (solid blue line). The same neurons' results in $\mathrm{SMT}_{\text {pre }}$ are plotted as a solid cyan line. Dashed blue line and dashed cyan line indicate the results of the same analysis in the standard dual-task and $\mathrm{SMT}_{\text {pre, }}$, respectively (both $n=51$, the curves are the same as in Fig. 8f). Horizontal dashed lines indicate the proportion expected by chance $(5 \%)$.

Supplementary Figure 12 Comparison of spatial selectivity between the SMT and DMT conditions. (a) Comparison of behavioral performance between the $\mathrm{SMT}_{\text {pre }}$ sessions with low percent correct rates and the DMT (standard dual-task) sessions with high percent correct rates. To perform this analysis, session-by-session percent correct rates in each memory task condition were rank-ordered and split at the median. The bottom half of $\mathrm{SMT}_{\text {pre }}$ sessions and the top half of DMT sessions were selected. This analysis included 51 sessions where spatially-selective delay-period activity was recorded in $\mathrm{SMT}_{\text {pre }}$. Data from the three DMT conditions in the standard dual-task (DMT-Up, DMT-Down and DMT-FR ${ }_{\text {std }}$ ) were collapsed. The sessions from the individual monkey were separately rank-ordered to avoid a biased subsampling from one 
monkey. The subsampled sessions gave highly similar percent correct rates between $\mathrm{SMT}_{\text {pre }}$ and

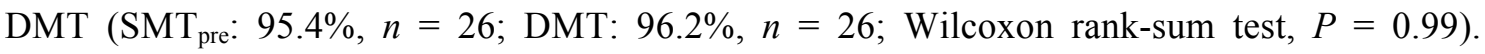
Conventions as in Fig. 2c. (b) Time course of $\mathrm{PEV}_{\text {attention }}$ (magenta), $\mathrm{PEV}_{\text {memory }}$ (blue), and $\mathrm{PEV}_{\text {interaction }}$ (green) in the standard dual-task for the 26 subsampled sessions. The magnitude of $\mathrm{PEV}_{\text {memory }}$ during the delay-period $(D)$ was significantly attenuated relative to that in $\operatorname{SMT}_{\text {pre }}(n$ $=26$, dashed cyan line) (Wilcoxon rank-sum test, $P=0.03$ ). Conventions as in Fig. 8. (c) Comparison of $\mathrm{PEV}_{\text {memory }}$ between the pre- $\mathrm{T}_{\mathrm{col}}$ change period and the follow-up fixation period in the standard dual-task. Following the conclusion of the attention task events, $\mathrm{PEV}_{\text {memory }}$ in the standard dual-task exhibited significant reawakening. (d) Comparison of $\mathrm{PEV}_{\text {memory }}$ and $\mathrm{PEV}_{\text {attention }}$ during the follow-up fixation period in the standard dual-task. The reawakening of $\mathrm{PEV}_{\text {memory }}$ during the follow-up fixation period coincided with the reprioritization of task processing between the attention and memory tasks. (e) Normalized population-averaged delay-period activity (grey shaded area) in the maximum (blue line) and minimum (red line) response locations in $\mathrm{SMT}_{\text {pre }}$ and the three DMT conditions in the standard dual-task for the subsampled sessions. For comparing delay-period activity across the four conditions, behavioral performance in DMT-Up, DMT-Down and DMT-FR std $_{\text {were }}$ rank-ordered separately. The subsampled sessions gave similar percent correct rates across the four conditions $(P=0.14, n=$ 26 for each of the four conditions,). For each neuron, firing rate in each 50-ms bin was divided by the peak delay-period firing rate at the maximum response location in the $\mathrm{SMT}_{\text {pre }}$ condition. Compared with $\mathrm{SMT}_{\text {pre }}$, the difference in activity between the maximum and minimum response

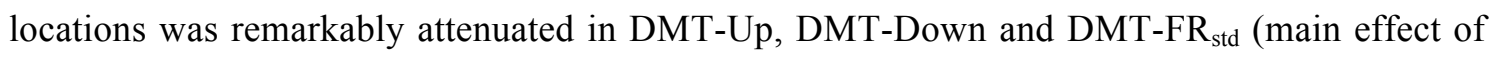
Task condition, $F_{3,100}=0.15, P=0.93$; Cue location, $F_{1,100}=52.95, P<10^{-4}$; interaction, $F_{3,100}=$ 6.25, $P=6 \times 10^{-4}$; two-way mixed design ANOVA). Conventions as in Fig. 5c. (f) Comparison of PEV values of the delay-period activity between the SMT and three DMT conditions. The three DMT conditions in the standard dual-task exhibited attenuation in spatial selectivity relative to $\mathrm{SMT}_{\text {pre }}$ (Kruskal-Wallis test, $P=0.03$ ). Conventions as in Supplementary Fig. 9d. 
Similar result was obtained when the rank-order of sessions was done over monkey-collapsed data (dotted line, $P=0.03)$. 


\section{a 京都大学}

A Self-archived copy in

(1 s)

Kyoto University Research Information Repository

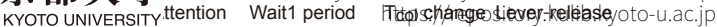

$\left.\begin{array}{|l|l|l|l}\hline 0 \\ 0 \\ 0\end{array}\right]$

Long trial $(40 \%)$

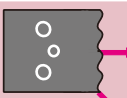

$(0.4 \mathrm{~s})$ $(<0.6 \mathrm{~s})$

0
0
0
0

Short trial $(60 \%)$

C Attention task : Short trial

Attention Wait1 period $\mathrm{T}_{\text {col }}$ change Lever-release cue $(0.4 \mathrm{~s}) \quad(2-5 \mathrm{~s}) \quad(0.4 \mathrm{~s}) \quad(<0.6 \mathrm{~s})$

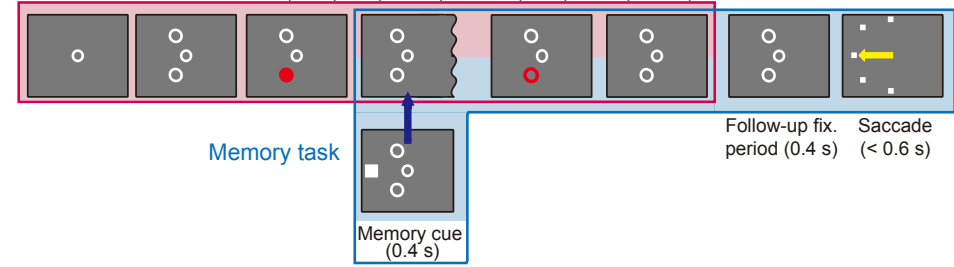

d Attention task : Long trial

Attention Wait1 period Catch change Wait2 period $\mathrm{T}_{\text {col }}$ change Lever-release $\begin{array}{llllll}\text { cue }(0.4 \mathrm{~s}) & (2-5 \mathrm{~s}) & (0.4 \mathrm{~s}) & (0.4-3.4 \mathrm{~s}) & (0.4 \mathrm{~s}) & (<0.6 \mathrm{~s})\end{array}$

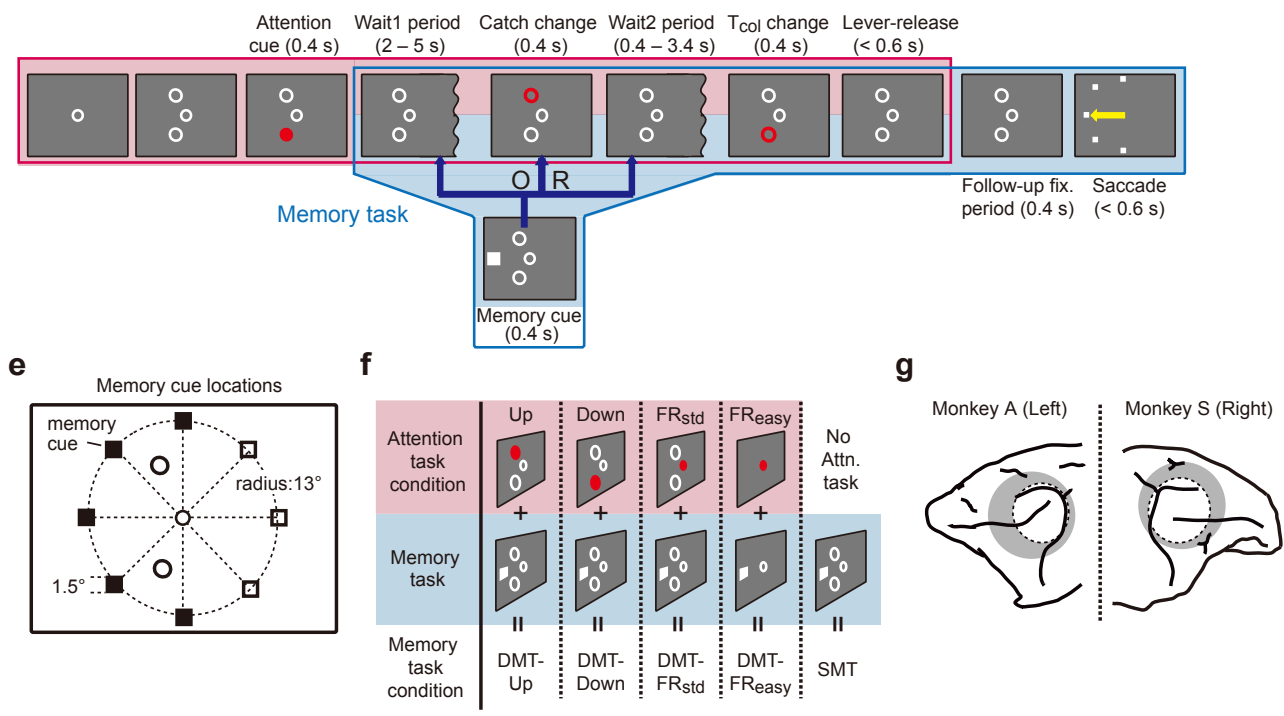

f

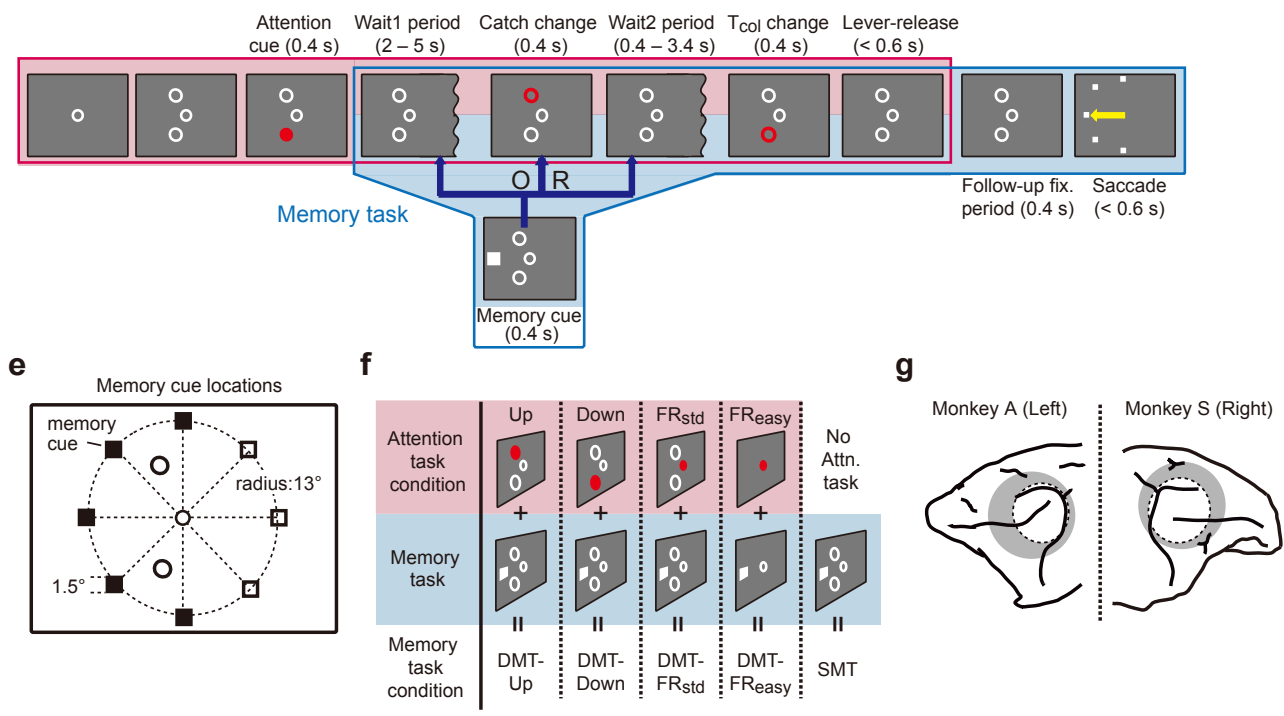

b $\quad$ Kyoto University Research Intormation Repository

Visual stimuli for Attention task

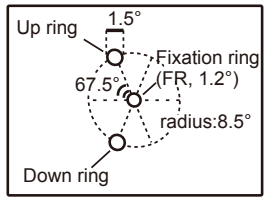

Catch change Wait2 period $\mathrm{T}_{\text {col }}$ change Lever-release $\begin{array}{llll}(0.4 \mathrm{~s}) & (0.4-3.4 \mathrm{~s}) \quad(0.4 \mathrm{~s}) \quad(<0.6 \mathrm{~s})\end{array}$

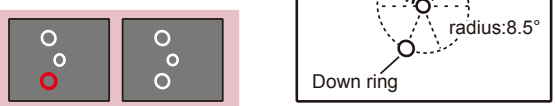


京都大学

Monkey A
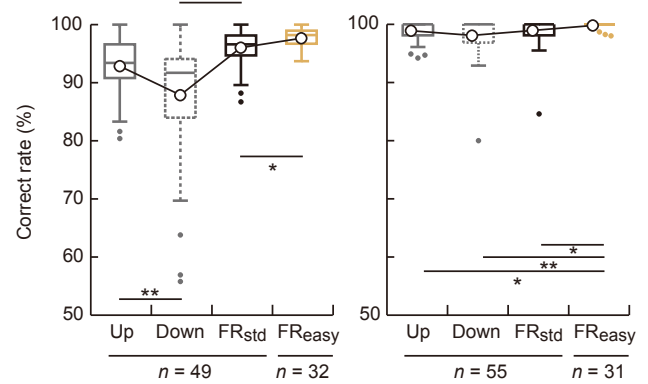

Attention task condition

d

Spatial proximity between attention and memoy cues

(e.g.) DMT-Up

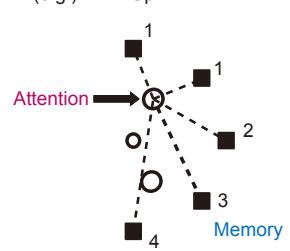

"Spatial-proximity" hypothesis of dual-task interference

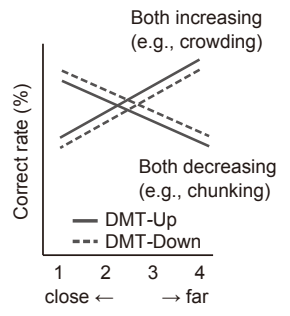

Spatial offset b Kyoto U MiøakìysResearch Information Raponkiteyra
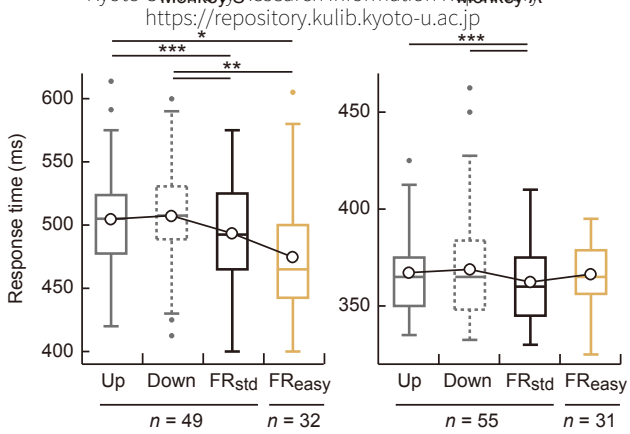

Attention task condition

e

Monkey S

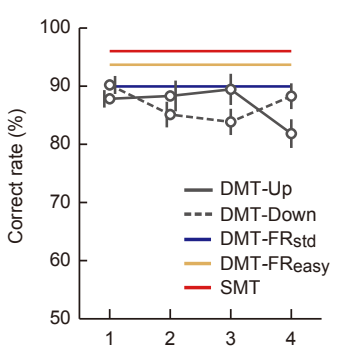

Spatial proximity between attention and memoy cues
Monkey A

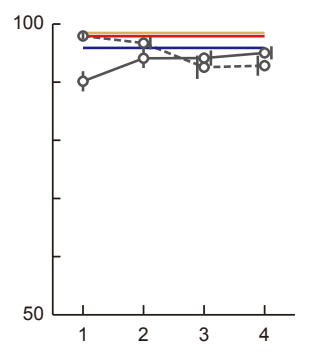

C

Monkey S

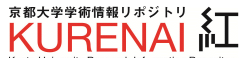

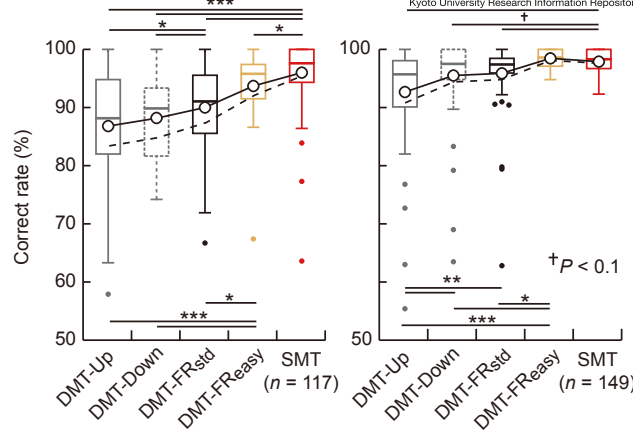

Memory task condition

\section{Figure-2 (Funahashi)}




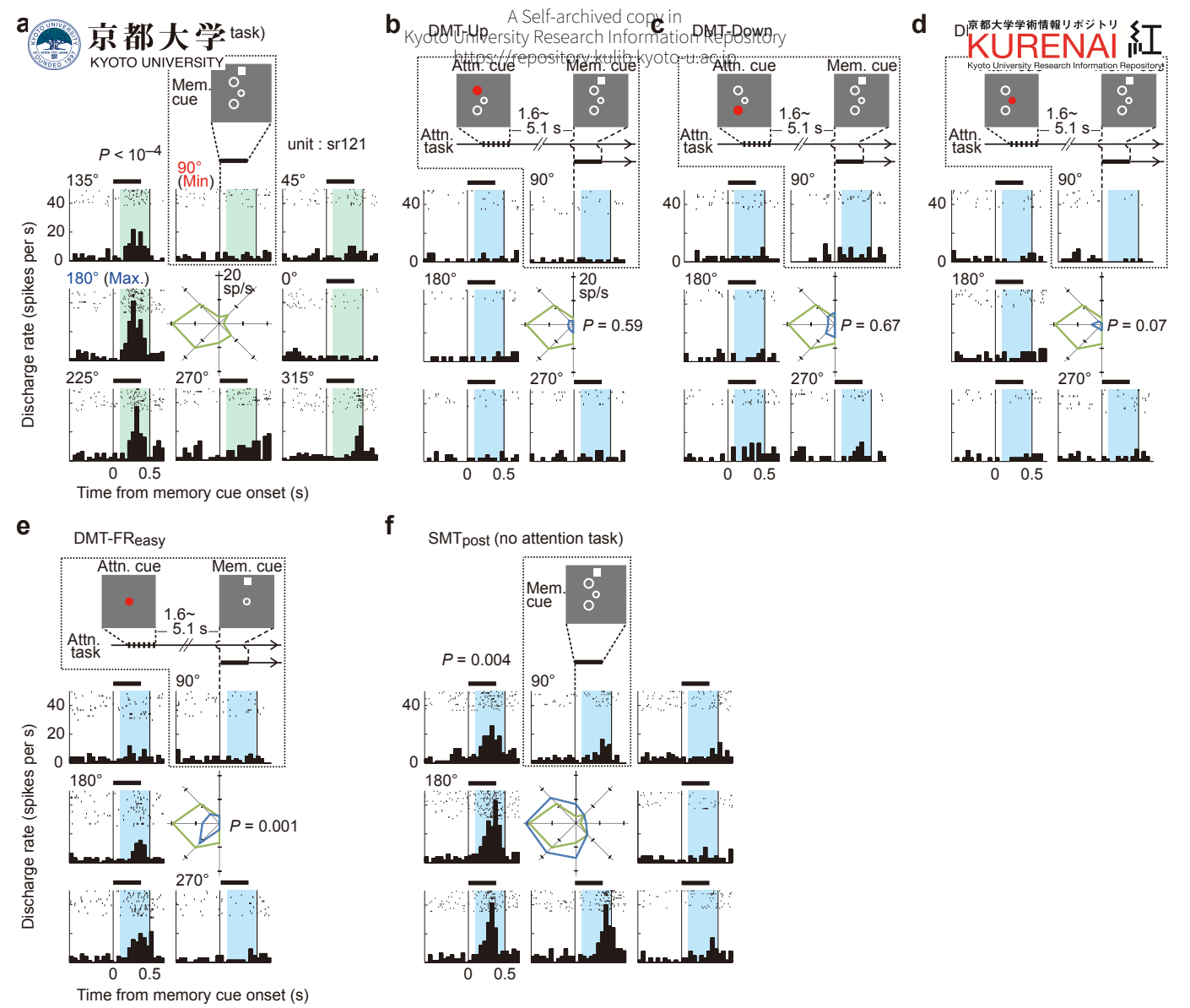

Figure-3 (Funahashi) 


\section{a}

京都大学。

KYTOTO UNIVERSITY
Attention

task No attention task

Memory

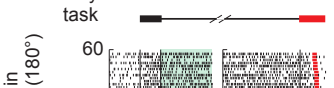

苋完

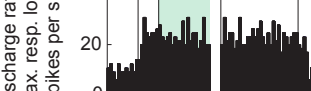

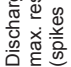

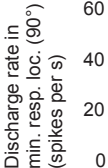

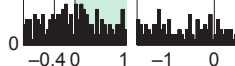

Time from event onset (s)

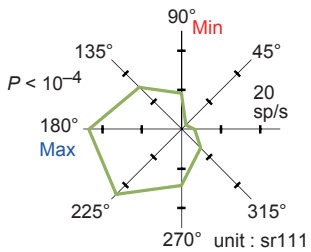

b

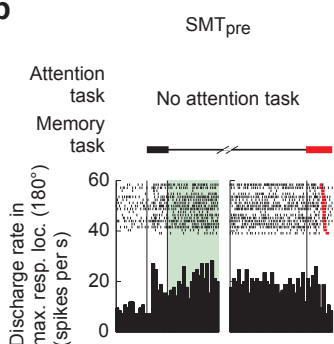

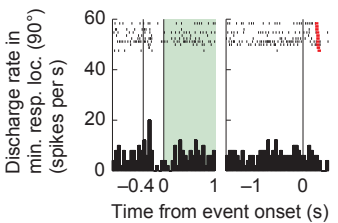

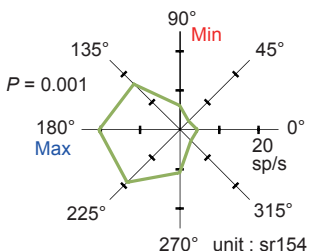

A Self-archived copy in

DMT-kpoto University ResearchDMMđғDowtion Repository https://repository.kulib.kyoto-u.ac.jp

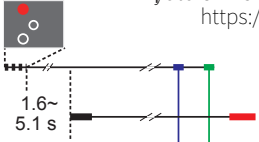

$["$ ind
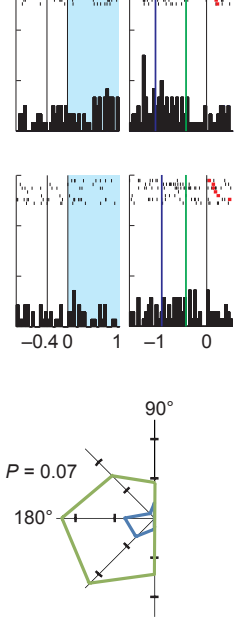

DMT-FReasy
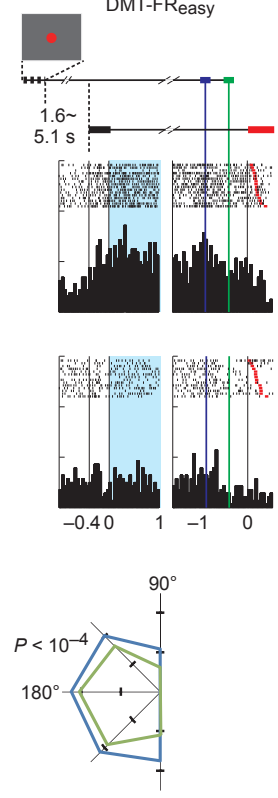

DMT-FR std

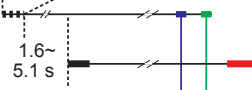

Find
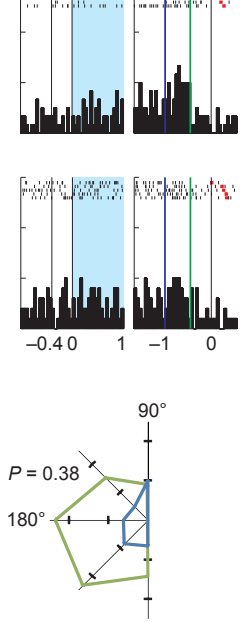

SMT post

No attention task

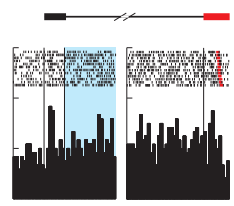

mint?
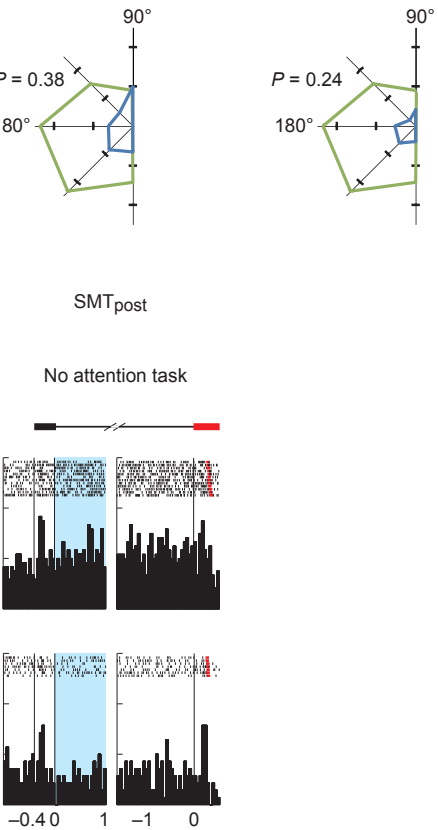
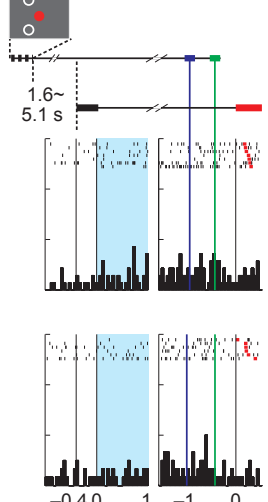

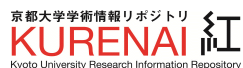

No attention task
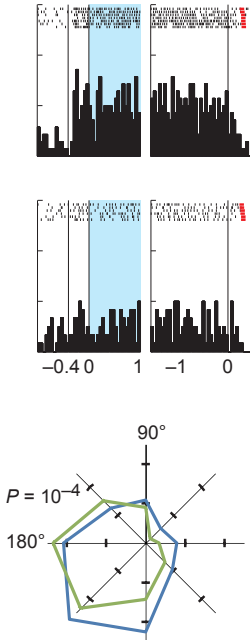


\section{A Self-archived copy in

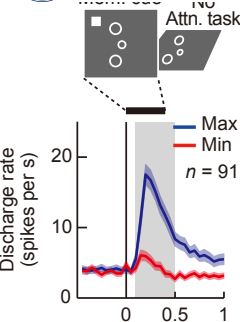

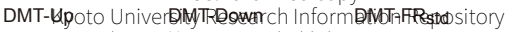
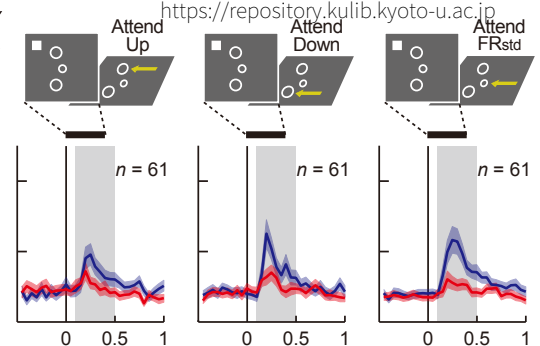

DMT-FR 都大学学術情報リポジトリ
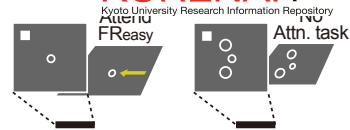

Time from memory cue onset (s)

b
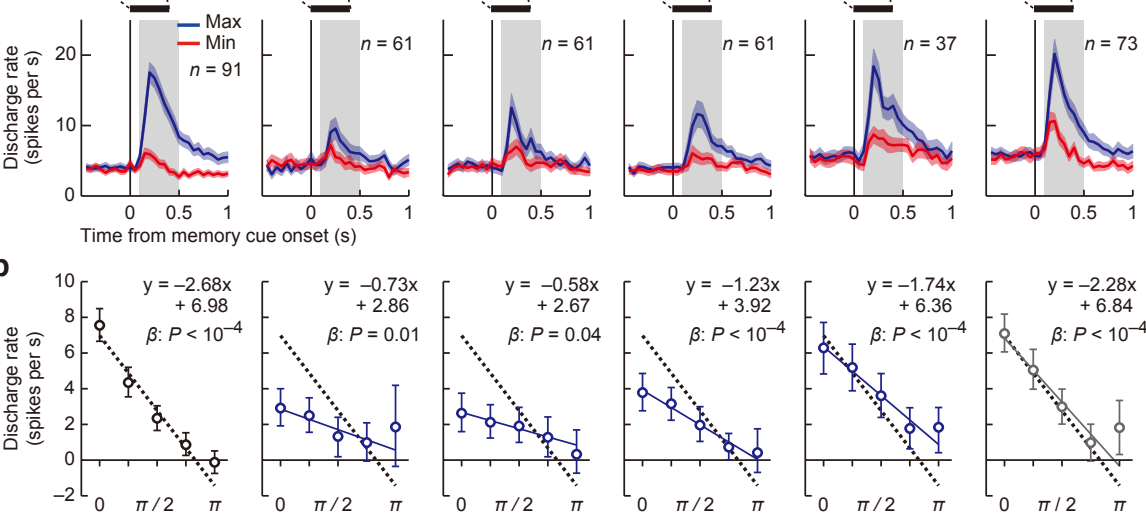

$\left(\operatorname{Max}\left(90^{\circ} \quad\left(180^{\circ}\right.\right.\right.$

loc.) apart) apart)

Angular distance of memory cue location relative to max. resp. location (rad)

C

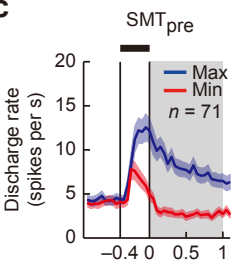

DMT-Up

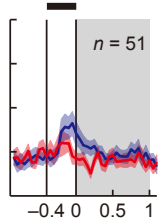

Time from delay-period onset (s)

d
范

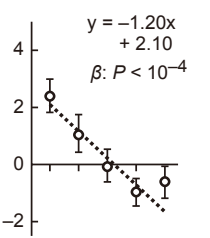

$0 \pi / 2 \pi$

$\left(\operatorname{Max}\left(90^{\circ}\left(180^{\circ}\right.\right.\right.$

loc.) apart) apart)
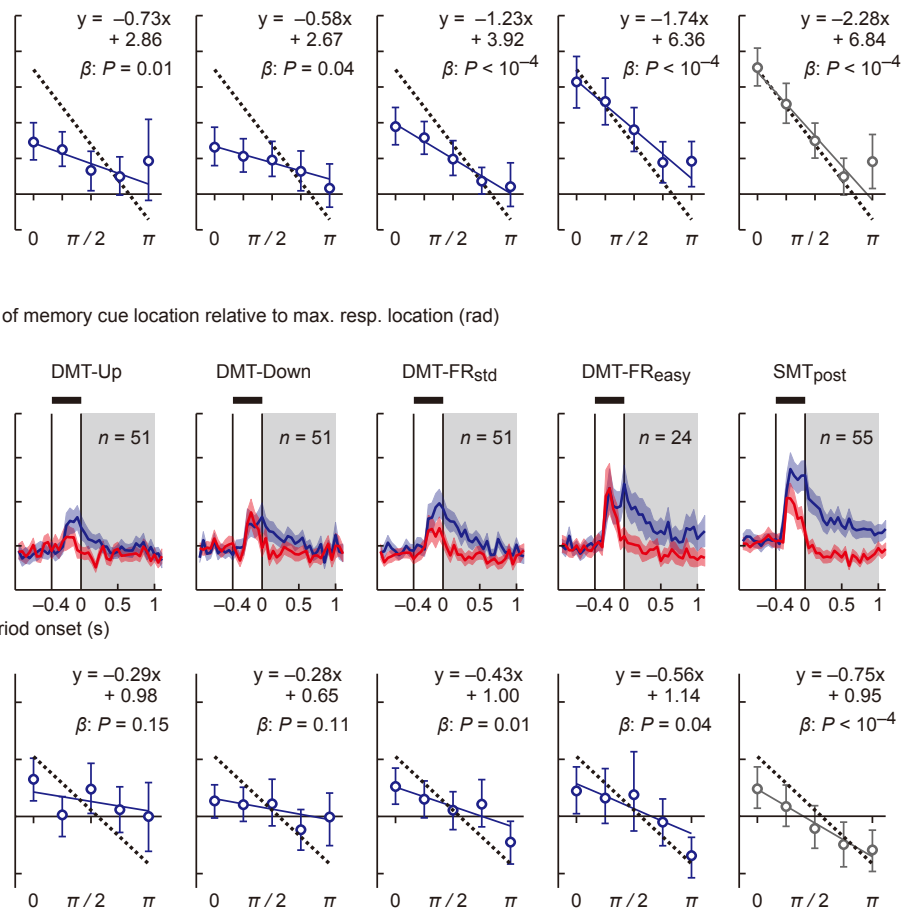

Angular distance of memory cue location relative to max. resp. location (rad) 
a

a
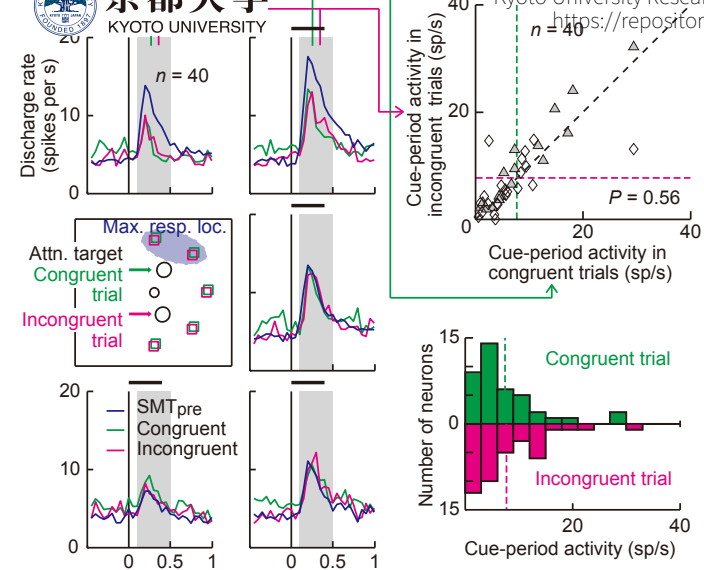

Time from memory cue onset (s)

C

Congruent trial vs. Incongruent trial

$10 \begin{array}{ll}n=40 & \text { Slope }: P=0.45 \\ & \text { Intercept : } P=0.03\end{array}$
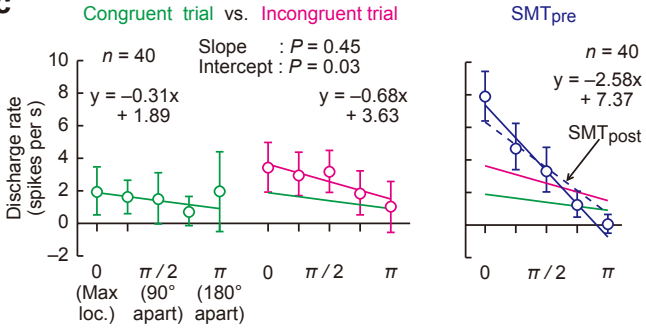

loc.) apart) apart)

Angular distance relative to max. resp. loc. (rad)
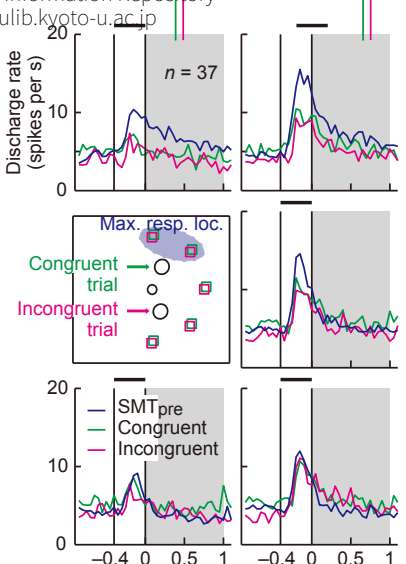

Time from delay-period onset (s)

d

Congruent trial vs. Incongruent trial

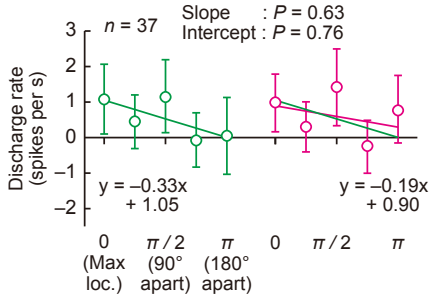

SMTpre

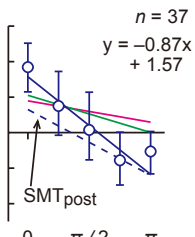

loc.) apart) apart)

Angular distance relative to max. resp. loc. (rad) 

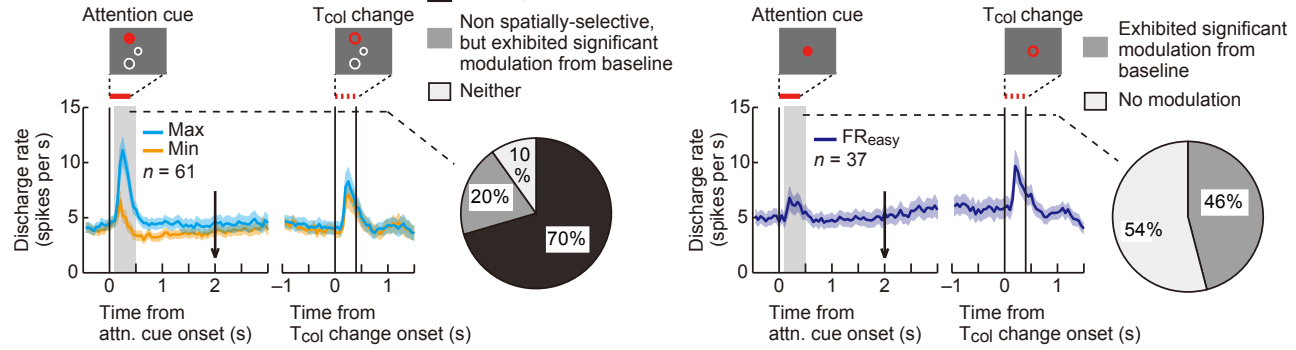

C

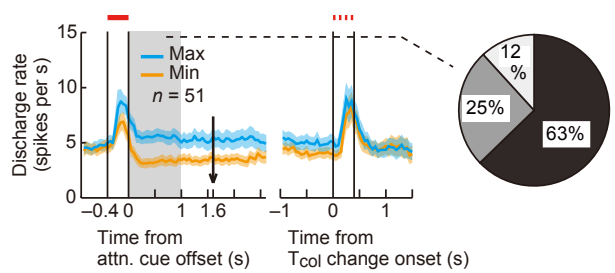

d

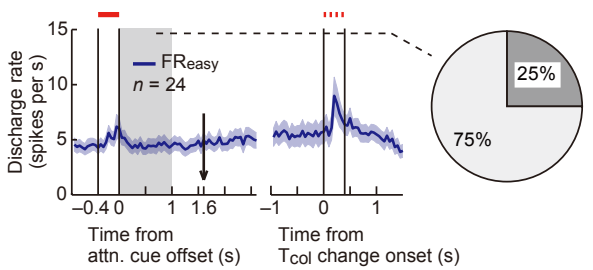

e
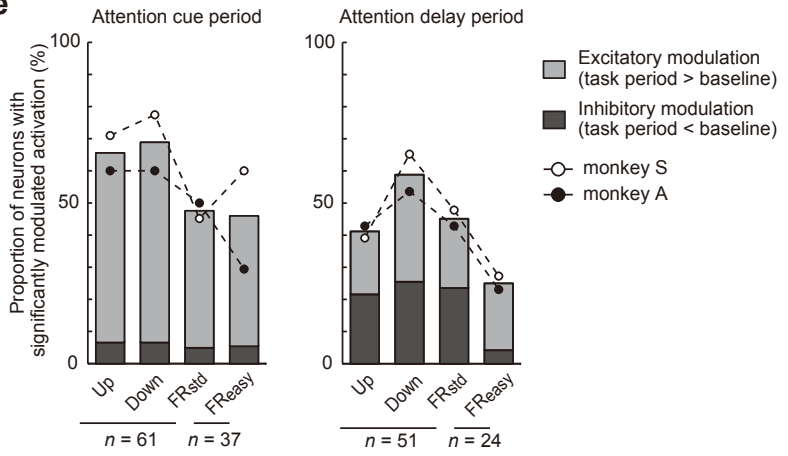

Attention task condition 


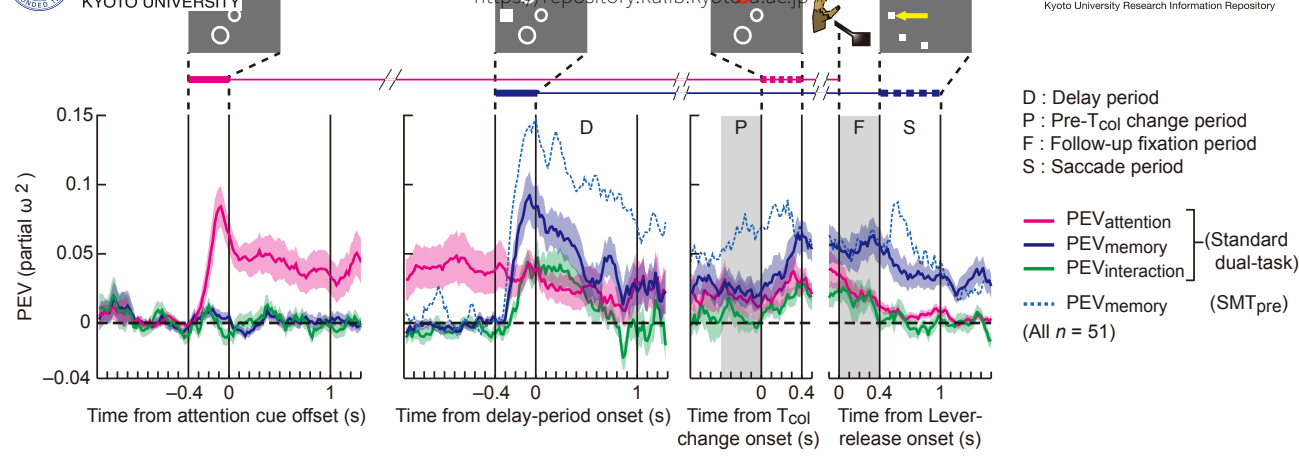

b

\section{$P E V_{\text {memory }}$ (standard dual-task) in} Pre- $T_{\text {col }}$ change period $(P)$ vs. Follow-up fix. period $(F)$

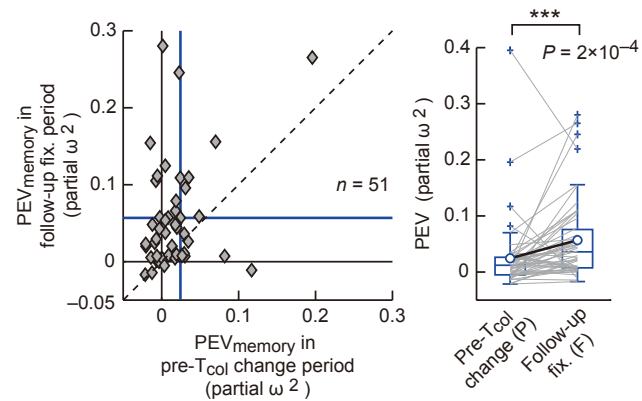

d

PEV memory (SMTpre) vs. PEV memory (standard dual-task) in Follow-up fixation period $(F)$
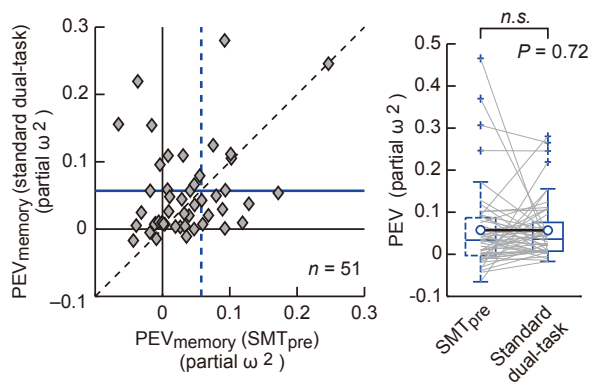

f
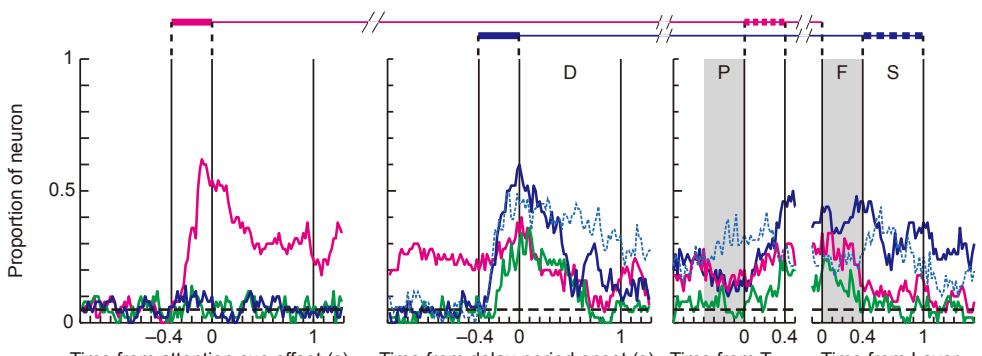

Factor:

- Attn. cue loc. $]$ - (Standard

— Interaction dual-task)

..... Mem. cue loc. (SMTpre) (All $n=51$ )

\section{Figure-8 (Funahashi)}




\title{
Supplementary Information
}

Neural mechanisms of dual-task interference and cognitive capacity limitation in the primate lateral prefrontal cortex

\author{
Kei Watanabe \& Shintaro Funahashi
}

Supplementary Figures $1-12$

Correspondence to:

Shintaro Funahashi, Ph.D.

Professor

Kokoro Research Center

Kyoto University

46 Shimoadachi-cho, Yoshida Sakyo-ku,

Kyoto, 606-8501, Japan

Phone \& Fax: (+81) 75-753-9674

E-mail: funahashi.shintaro.2z@kyoto-u.ac.jp 


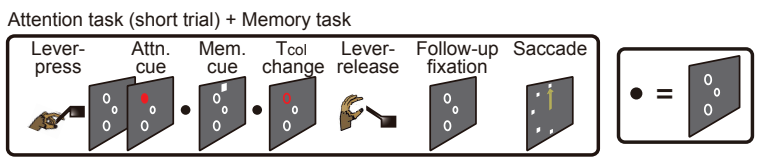

Attention task (long trial) +Memory task

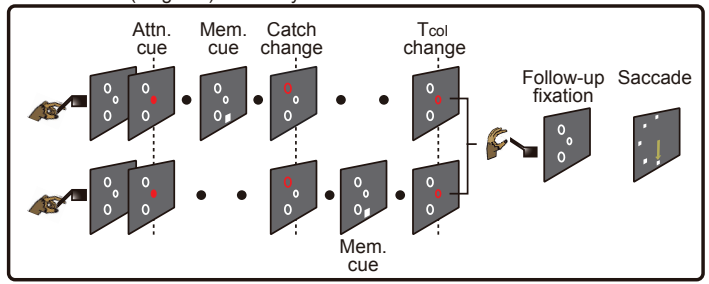

b Easy dual-task

Attention task (short trial) + Memory task

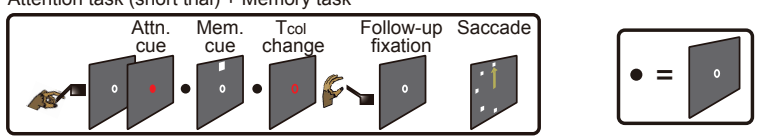

Attention task (long trial) + Memory task

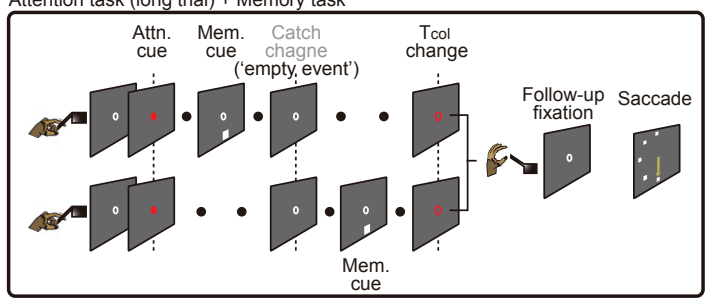

c Single memory task (SMT)

Memory task + No Attention task (all 'empty events', short trial)

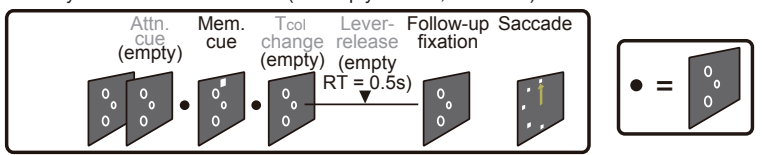

Memory task + No Attention task (all 'empty events', long trial)

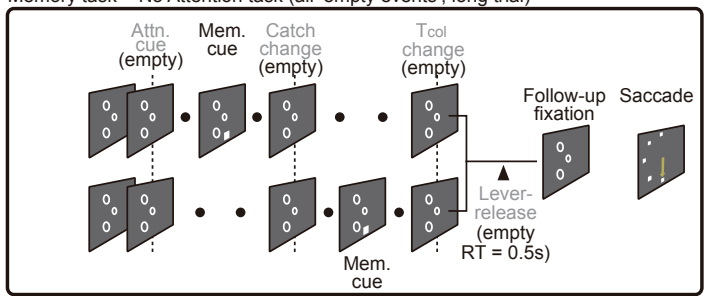

Supplementary Figure 1 Event sequence of example trials. (a) Example trials in the standard dual-task. The upper row depicts an example dual-task trial in which the attention task is performed as a short trial. The bottom row shows a trial in which the attention task is performed as a long trial. Note that, in the long trial, there were two possible patterns in the temporal order of catch change and memory cue presentation. (b) Same as in panel (a), but for the easy dual-task. Note that, in the long trial (bottom row), catch change was scheduled and executed as an 'empty event'. (c) Example trials in the single memory task (SMT). The time course of the task was matched with that in the standard and easy dual-tasks. However, while all attention task events were scheduled, they were executed as 'empty events'. Trials were automatically initiated by the appearance of FR after an intertrial interval (4.0-7.0 s). 
Monkey A

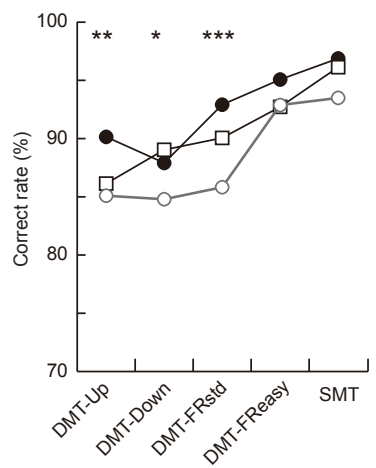

b

Monkey S

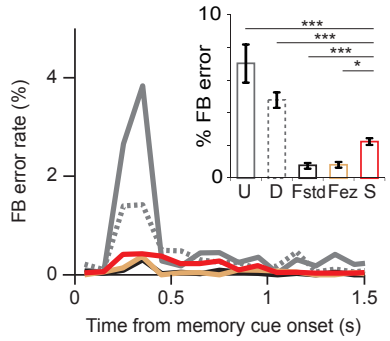

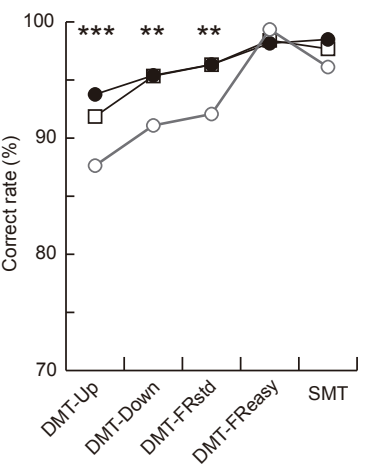

Monkey A

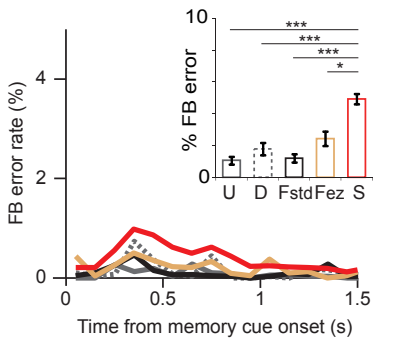

- Short delay (<2s)

$\neg-$ Medium delay $(2-4 \mathrm{~s})$

$-\infty$ Long delay $(>4 \mathrm{~s})$

* $\quad P<0.05$

** $P<0.01$

*** $P<0.001$

C

Monkey S

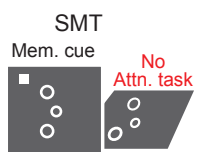

DMT-Up

DMT-Down
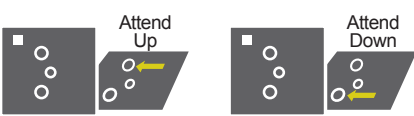

DMT-FRstd

DMT-FReasy

** $P<0.01$

*** $P<0.001$

(Steel-Dwass test)

DMT-Up (U)

"II!" DMT-Down (D)

- DMT-FRstd (Fstd)

DMT-FReasy (Fez)

$\begin{array}{r}P<0.05 \\ \hline * 0.01\end{array}$
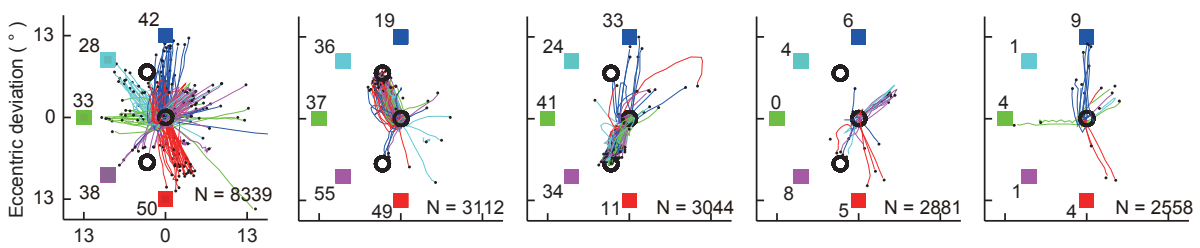

d Monkey A
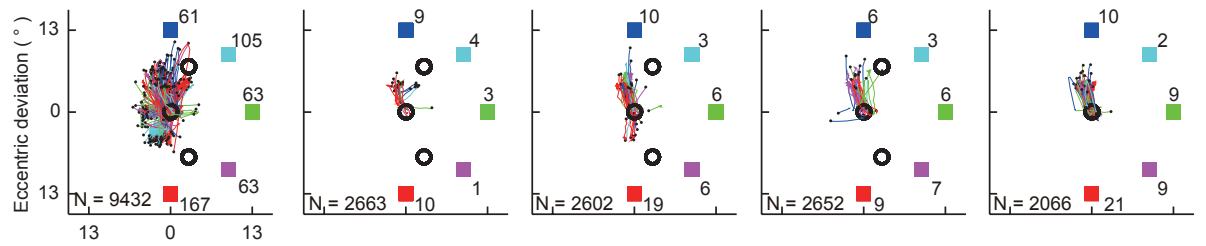
Supplementary Figure 2 Additional evidence supporting the presence of dual-task interference effect in the DMT conditions. (a) Mean percent correct rates in the SMT and four DMT conditions plotted separately for the trials with short $(<2.0 \mathrm{~s})$, medium $(2.0-4.0 \mathrm{~s})$ and long $(>4.0 \mathrm{~s})$ memory delay periods. In monkey $\mathrm{S}(\mathrm{left}), \mathrm{a}$ two-way mixed-design ANOVA showed significant main effects of both Task condition and Delay length $(P<$ $\left.10^{-4}\right)$, and a nonsignificant interaction effect $(P=0.27)$. In monkey A (right), there were significant main effects of both Task condition and Delay length $\left(P<10^{-4}\right)$, and an interaction effect $(P=0.02)$. Asterisks indicate the result of the simple effect ANOVA for the factor Delay length. (b) Time course of FB error rates relative to memory cue onset averaged across all sessions for monkeys S (left) and A (right). Inset bar graphs indicate the mean FB error rate during the 1-s period following memory cue onset. Error bars indicate s.e.m. During this period, monkey $\mathrm{S}$ showed a significant increase in the FB error rate in DMT-Up and DMT-Down compared with SMT (post-hoc Steel-Dwass test, $P<10^{-4}$; omnibus Kruskal-Wallis test, $P<10^{-4}$ ), indicating that the oculomotor aspect of memory task performance was substantially interfered with by the concurrent attention task. (c, d) Trajectories and end points of FB eye movements that occurred during the 1-s period following memory cue onset in monkeys S (c) and A (d). Three unfilled black circles indicate the ring stimuli for the attention task. Colors are assigned to each memory cue location (square) and each FB eye movement trajectory so that the color of a given FB eye movement trajectory indicates the location of the memory cue presentation that preceded this FB error. End points of FB eye movements are shown as black dots. Numbers shown on each colored square indicate the cumulative number of FB errors across sessions in each memory cue location. The total number of trials $(\mathrm{N})$ in which the memory cue was presented is shown at the bottom of each panel. In the DMT-Up and DMT-Down conditions in monkey S, regardless of memory cue location, FB eye movements after memory cue onset were predominantly directed toward the attention target ring, rather than the memory cue location as observed in SMT. However, importantly, at the time of memory cue onset, the attention cue had been removed from the monitor for 1.6-5.1 s, and the monkeys were simply viewing the still images of three rings. This indicates that around the time of memory cue presentation, information regarding the position of the target ring for the attention task was occupying monkey S's processing capacity, suggesting that the level of readiness for memory cue encoding was severely disrupted. Monkey A's FB errors were characterized by short eye movements clustered on the vertical axis, regardless of the memory task conditions. The similarity in FB eye movement trajectories between the SMT and DMT conditions indicates that in all of the four DMT conditions, the preparatory state for memory cue encoding was not disrupted by the concurrent attention task. Thus, we concluded that, for monkey A, dual-task interference on the oculomotor aspect memory task performance was minimal. 
a

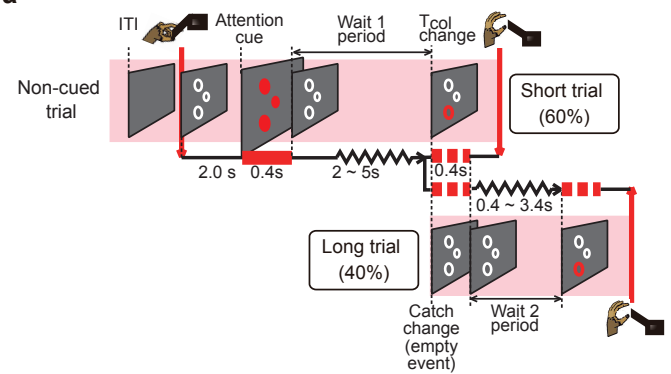

c

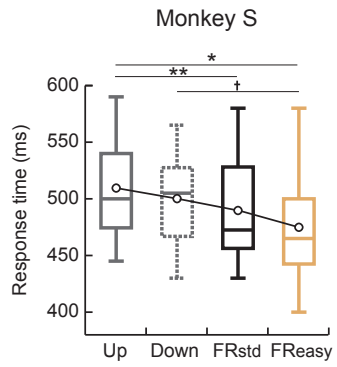

Attention task condition (in cued trials only)

e

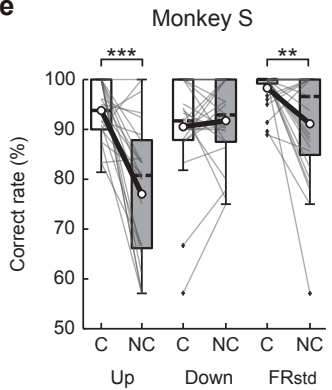

b

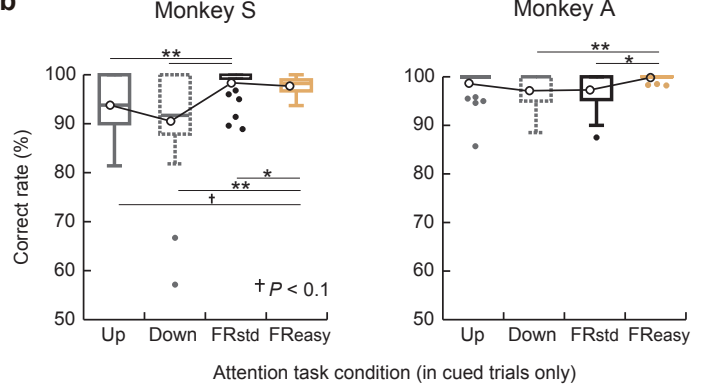

d

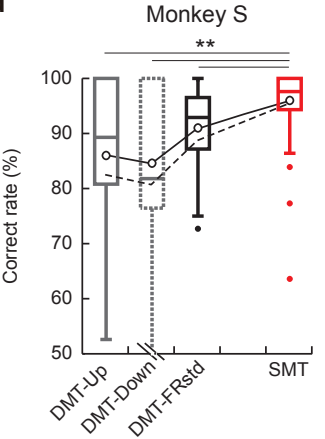

Memory task condition (in cued trials only)

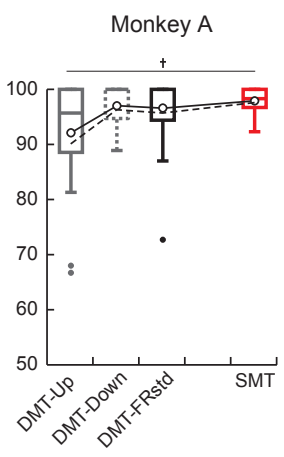

Monkey A

f

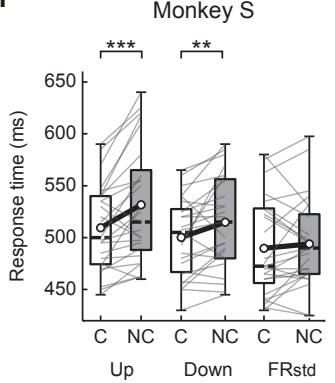

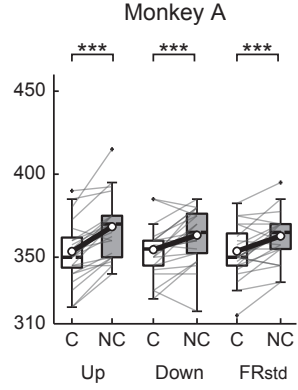

Trial type in each attention task condition (C:cued trial, NC: non-cued trial) 
Supplementary Figure 3 Behavioral performance in the modified standard dual-task. (a) Schematic diagram of the event sequence for non-cued trials of the attention task that were randomly inserted among the Up, Down, $\mathrm{FR}_{\text {std }}$ conditions (cued trials). A catch change was scheduled but executed as an 'empty event' without actual changes in the display items. (b) Distribution of the session-by-session percent correct rates in the attention task in the modified standard dual-task (consecutive 25 sessions in each monkey) in monkeys S (left) and A (right). Only data from cued trials are shown. The results in $\mathrm{FR}_{\text {easy }}$ (Fig. 2a) are also shown in the rightmost box plot. The statistical testing procedure and conventions were the same as in Fig. 2a. The results in FR easy $($ Fig. 2b) are also shown in the rightmost box plot. (c) Distribution of the session-by-session median RTs in the attention task for monkeys S (left) and A (right). (d) Distribution of the session-by-session percent correct rates in the three DMT conditions for monkeys S (left) and A (right). The results in SMT (Fig. 2c) are also shown in the rightmost box plot. The dotted line indicates the mean percent correct rates after "corrected-for-guessing" transformation. $P$-values were adjusted for three multiple comparisons between the SMT and three DMT conditions. (e) Comparison of the session-by-session percent correct rates between cued (C) and non-cued (NC) trials in the attention task. In monkey $\mathrm{S}$, the percent correct rates in cued trials were significantly higher than those in non-cued trials (two-way repeated-measures ANOVA: main effect of Cueing, $P=2 \times 10^{-4}$; Attention condition, $P<10^{-4}$; interaction, $P<10^{-4}$ ) (simple main effect of Cueing: $\mathrm{Up}, P<10^{-4}$; $\mathrm{FR}_{\mathrm{std}}, P=0.006$; both $\mathrm{C}$ $>$ NC). (f) Comparison of the session-by-session median lever-release RTs between cued (C) and non-cued (NC) trials in the attention task. In both monkeys, the RTs in cued trials were significantly shorter than those in non-cued trials (monkey S: main effect of Cueing, $P=5 \times 10^{-4}$; Attention condition, $P<10^{-4}$; interaction, $P=$ 0.06; monkey A: main effect of Cueing, $P<10^{-4}$; Attention condition, $P=0.17$; interaction, $P=0.09$ ) (simple main effect of Cueing: $P<0.009$ for the Up and Down conditions in monkey $\mathrm{S} ; P<2 \times 10^{-4}$ for all three conditions in monkey A; all $\mathrm{NC}>\mathrm{C}$ ). 

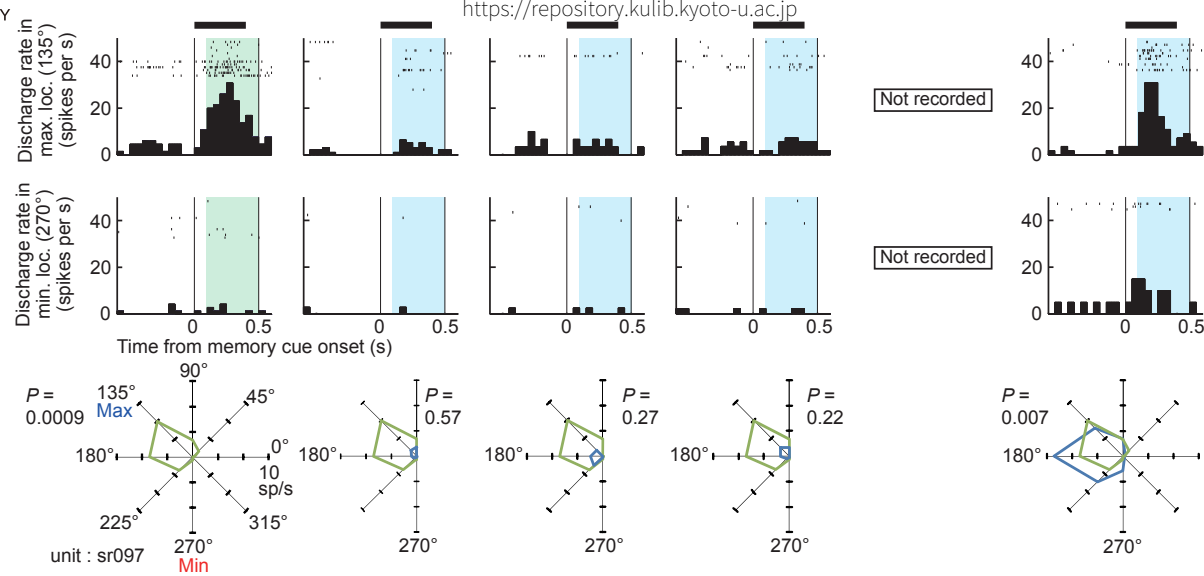

Not recorded

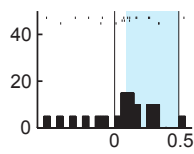

b
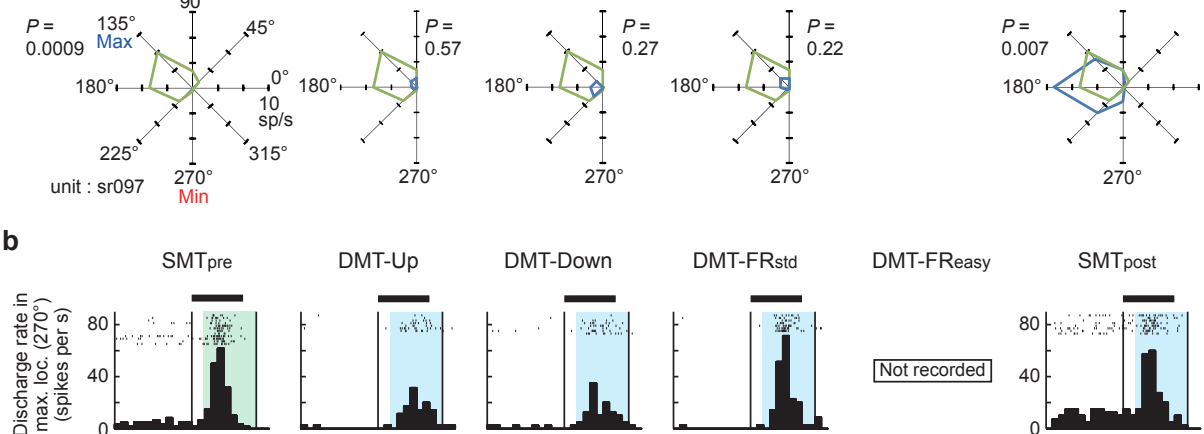

DMT-Down

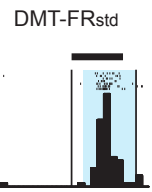

DMT-FReasy

SMTpost
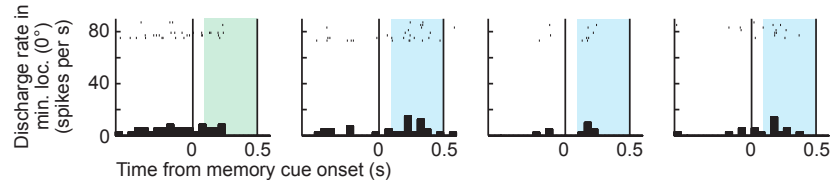

Not recorded

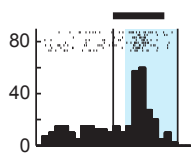

Not recorded
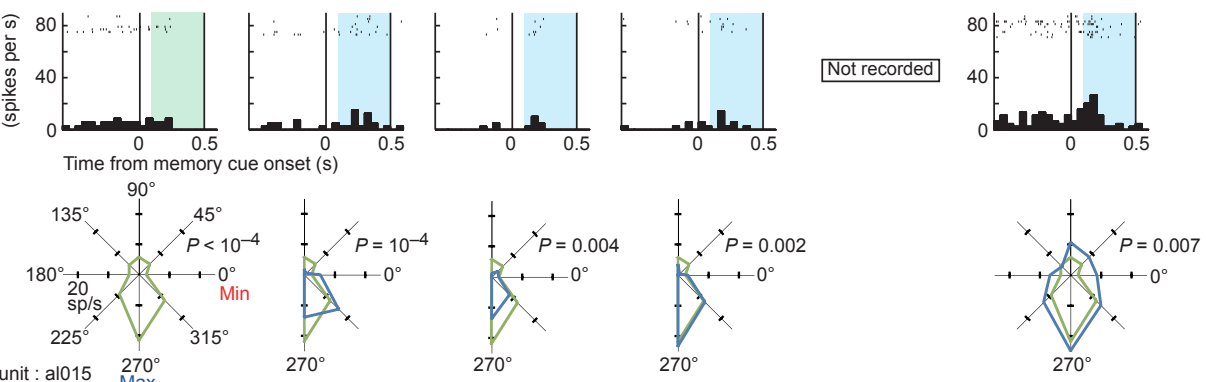

C

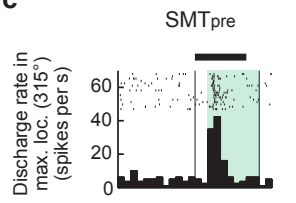

DMT-Up

DMT-Down

DMT-FRstd

DMT-FReasy

SMTpost

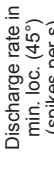

Not recorded

Not recorded

Not recorded
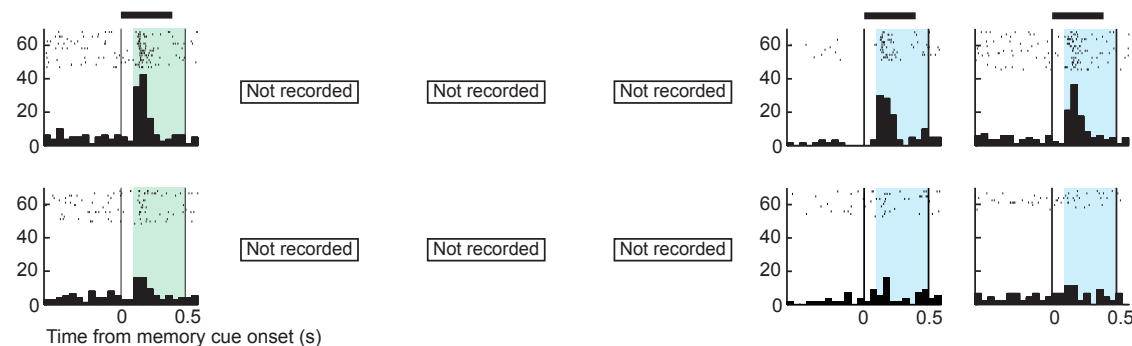

Not recorded

Not recorded

Not recorded
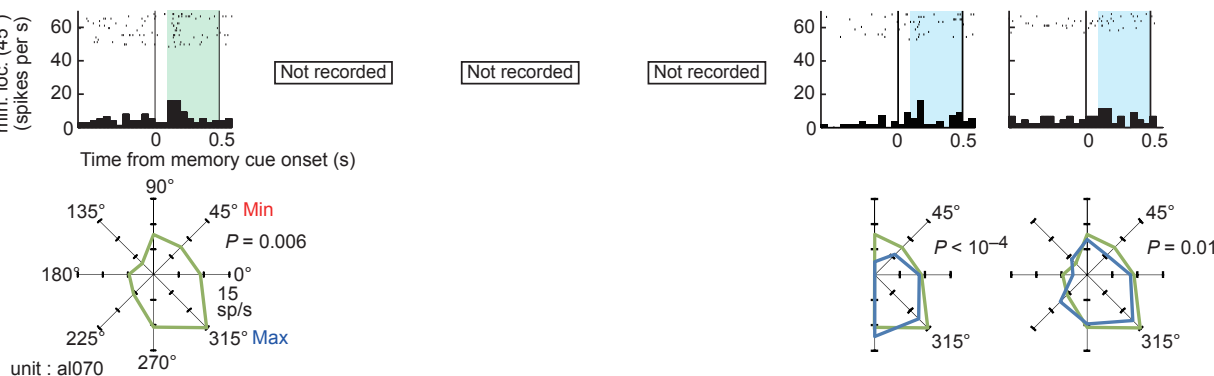

Supplementary Figure 4 Cue-period activity of example neurons. (a) Activity of a single neuron (monkey S, right hemi.) recorded in the $\mathrm{SMT}_{\text {pre, DMT-Up, DMT-Down, DMT-FR }}$ (std (standard dual-task), and SMT $\mathrm{S}_{\text {post }}$ conditions. Conventions as in Fig. 4. (b) Same as in panel (a), but for a neuron recorded from monkey A (left hemi.) This neuron exhibited significant spatial selectivity in all three DMT conditions in the standard dual-task. However, the strength of cue-period activity in the maximum response location $\left(270^{\circ}\right)$ is significantly attenuated in DMT-Up and DMT-Down compared to that in $\mathrm{SMT}_{\text {pre }}$ (c) Activity of a single neuron (monkey A, left hemi.)

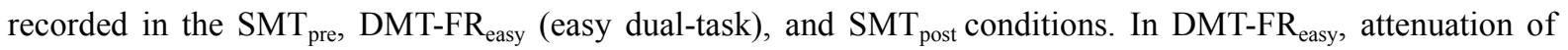
both the magnitude and selectivity of cue-period activity was absent. 
a

SMTpre

DMT-FReasy

SMTpost -1

DMT-Up

DMT-Down

DMT-FRstd

SMTpost -2
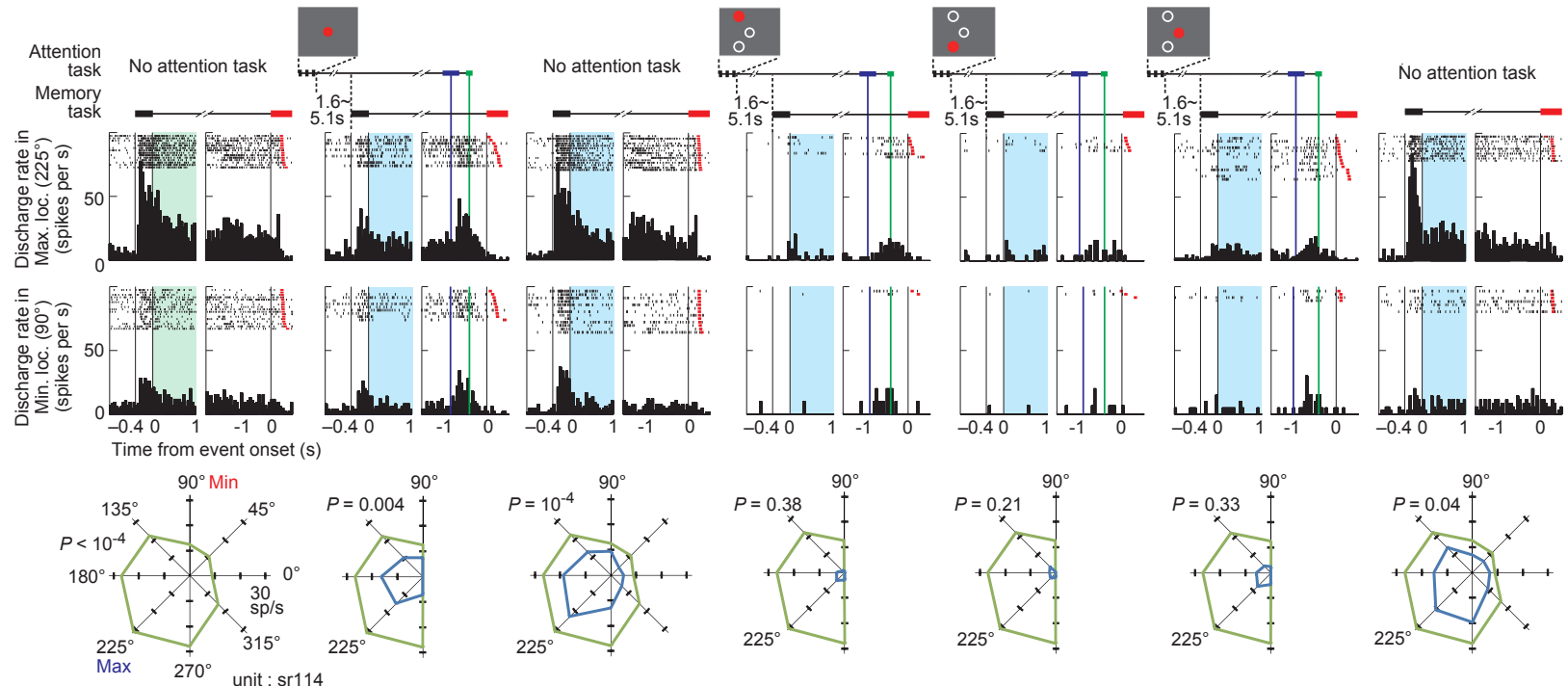

b

SMTpre

DMT-Up

DMT-Down

DMT-FRstd
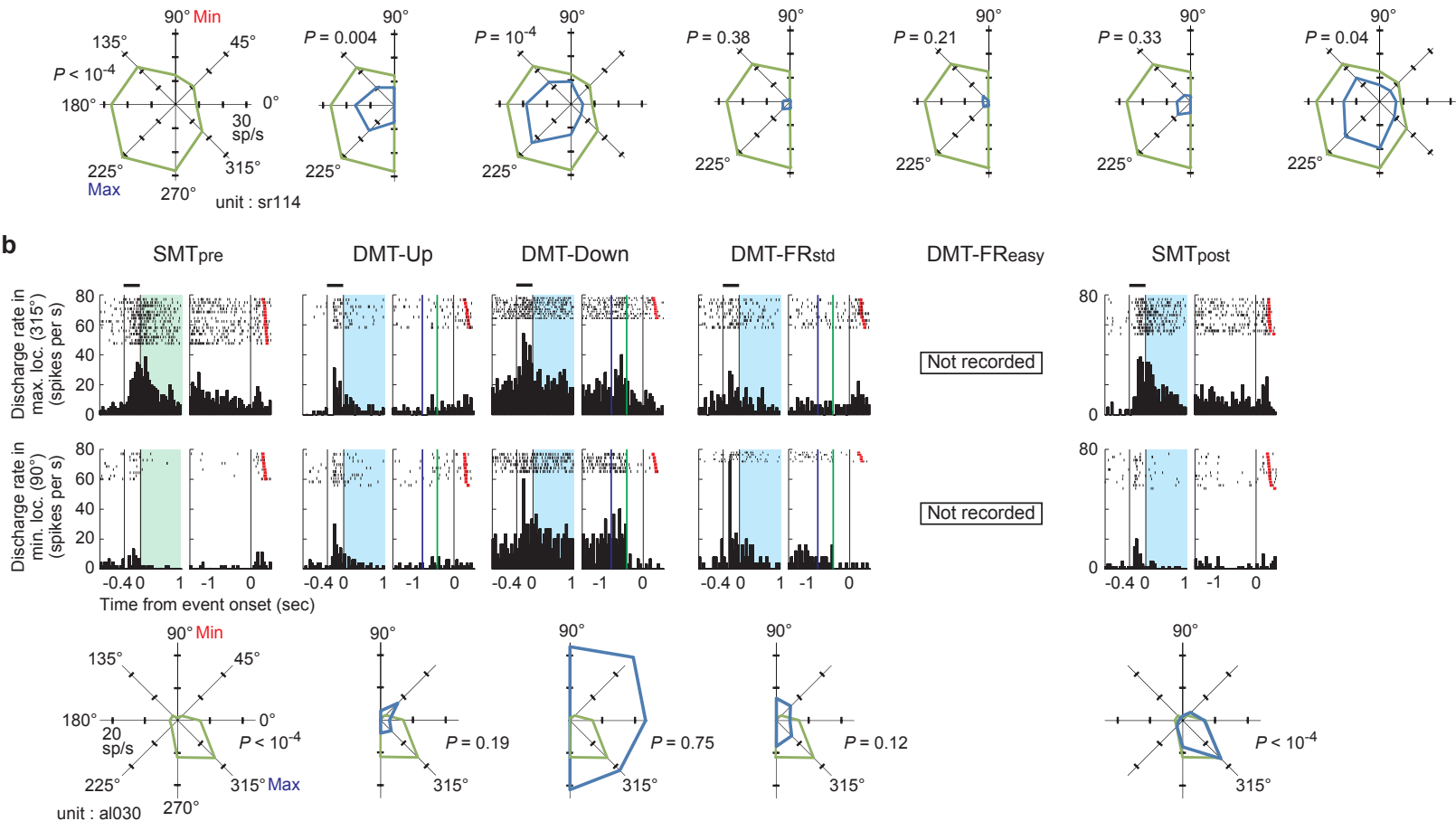

c

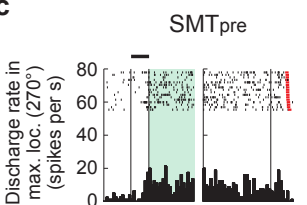

DMT-Up

DMT-Down

Not recorded

Not recorded

Not recorded

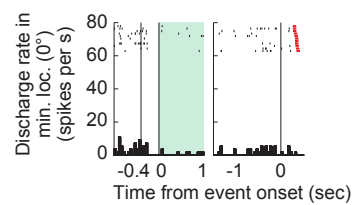

Not recorded

Not recorded

Not recorded

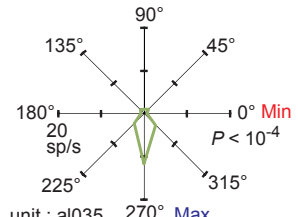

DMT-FRstd
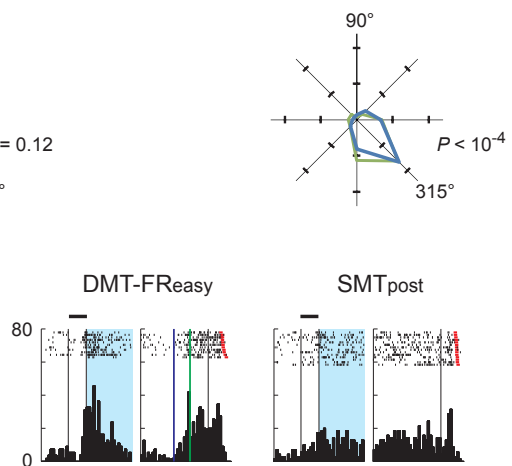
Supplementary Figure 5 Delay-period activity of example neurons. (a) Activity of a single neuron (monkey $\mathrm{S}$, right hemi.) recorded in the $\mathrm{SMT}_{\text {pre }}$, DMT-Up, DMT-Down, DMT-FR $\mathrm{std}_{\text {st }}$ DMT-FR easy, and $\mathrm{SMT}_{\text {post }}$ conditions Conventions as in Fig. 4. From left to right, seven memory task conditions, including two $\mathrm{SMT}_{\text {post }}$ blocks $\left(\mathrm{SMT}_{\text {post }}{ }^{-1}\right.$ and $\left.\mathrm{SMT}_{\text {post }}-2\right)$, are shown in the order of recording, except for DMT-Up, DMT-Down and DMT-FR $_{\text {std. }}$ For this and three other neurons (monkey $\mathrm{S}$ ) with spatially-selective delay-period activity in $\mathrm{SMT}_{\text {pre }}$ activities were obtained in both the standard and easy dual-tasks. (b) Activity of a single neuron (monkey A, left hemi.) recorded in $\mathrm{SMT}_{\text {pre }}$, DMT-Up, DMT-Down, DMT-FR std $_{\text {, and }}$ a $\mathrm{SMT}_{\text {post }}$ Note that in DMT-Down, the activity level was elevated in all memory cue locations because the attention cue had been presented near the neuron's maximum response location $\left(315^{\circ}\right)$. Nevertheless, spatial selectivity of delay-period activity was lost in this condition $(P=0.75)$. (c) Activity of a single neuron (monkey A, left hemi.) recorded in $\mathrm{SMT}_{\text {pre, }}$ DMT-FR easy, and $\mathrm{SMT}_{\text {post. }}$. This neuron exhibited delay-period activity similar to that in panel (b). However, attenuation of both the magnitude and selectivity of delay-period activity was absent in DMT-FR $\mathrm{easy}_{\text {. }}$ 

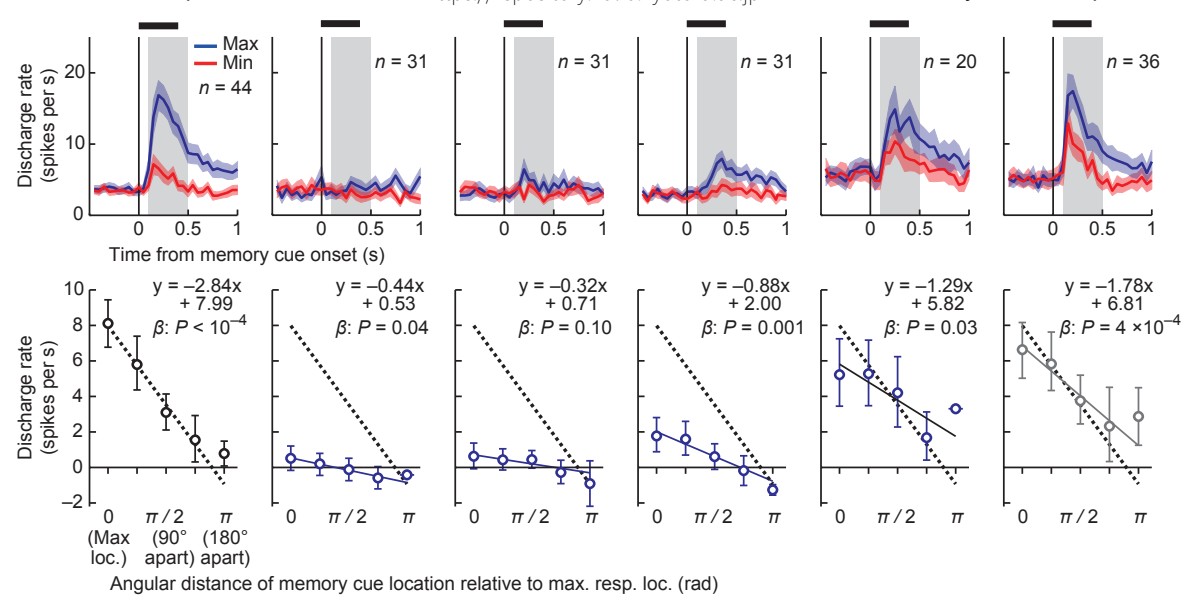

b

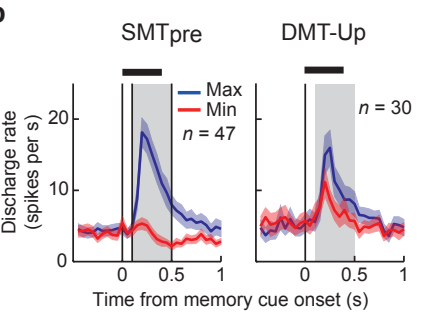

DMT-Down

DMT-FRstd

DMT-FReasy
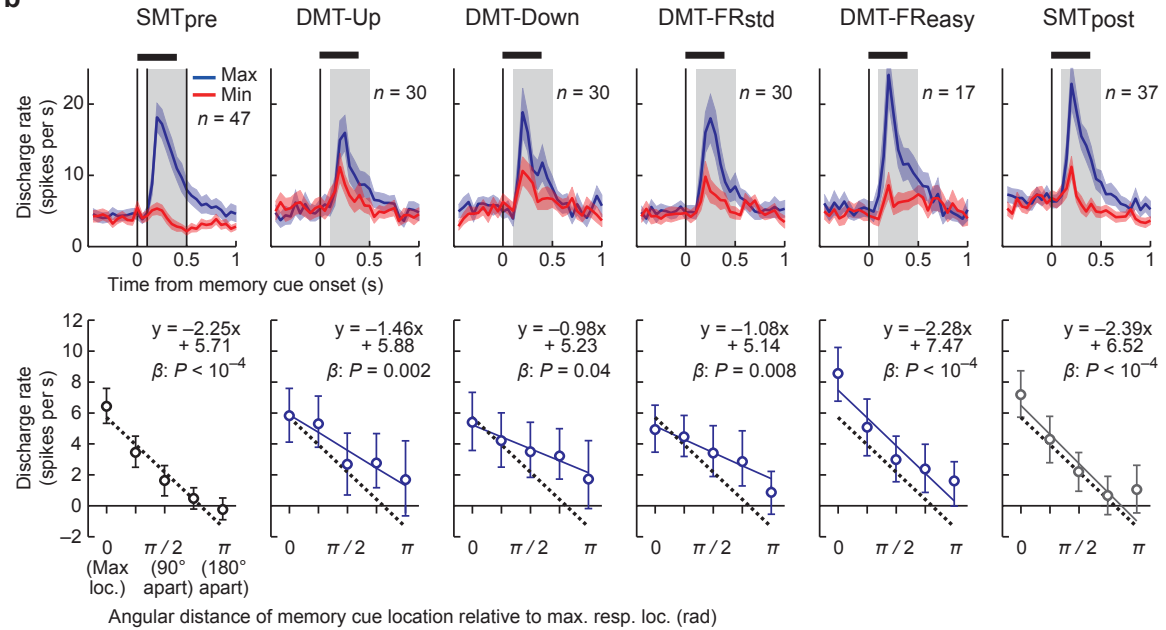

apart) apart)

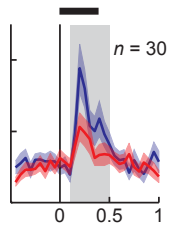

Supplementary Figure 6 Population cue-period activities of individual monkeys. (a, b) Data are for monkeys S (a) and A (b). Upper row: population cue-period activity in the maximum (blue line) and minimum (red line) response locations across the six memory task conditions. In both monkeys, significant interaction effects [Task condition $\times$ Cue location] were observed among cue-period activity (monkey S: main effect of Task condition, $F_{5,187}=10.85, P<10^{-4}$; Cue location, $F_{1,187}=114.88, P<10^{-4}$; interaction, $F_{5,187}=10.52, P<10^{-4}$; monkey A: main effect of Task condition, $F_{5,185}=0.54, P=0.76$; Cue location, $F_{1,185}=154.90, P<10^{-4}$; interaction, $F_{5,185}=$ 3.57, $P=0.004$; two-way mixed design ANOVA). Bottom row: population spatial tuning during the cue-period. Conventions as in Fig. 5. Although, in both monkeys, cue-period activity exhibited a significant attenuation of spatial selectivity under DMT, the degree of attenuation was smaller in monkey A, whose cue-period activity exhibited a robust increase following memory cue onset. This suggests that, in monkey A, information processing of the memory task was rather unaffected in the initial phase (time period immediately following memory cue presentation), whereas in monkey S, processing of the memory task was considerably disrupted from this phase. In agreement with this notion, comparison of the behavioral performance between the two monkeys showed that monkey A exhibited a more moderate dual-task interference on memory task performance than monkey S (Fig. 2c). This was particularly evident in the trials that had short memory delay period $(<2.0 \mathrm{~s})$ (Supplementary Fig. 2a). In addition, monkey A's oculomotor behavior following memory cue presentation did not show signs of dual-task interference, whereas monkey S's oculomotor behavior in the same epoch clearly exhibited interference by the attention task, as indicated by a significant increase in FB error rates in DMT-Up and DMT-Down relative to SMT (Supplementary Fig. 2b-d). 


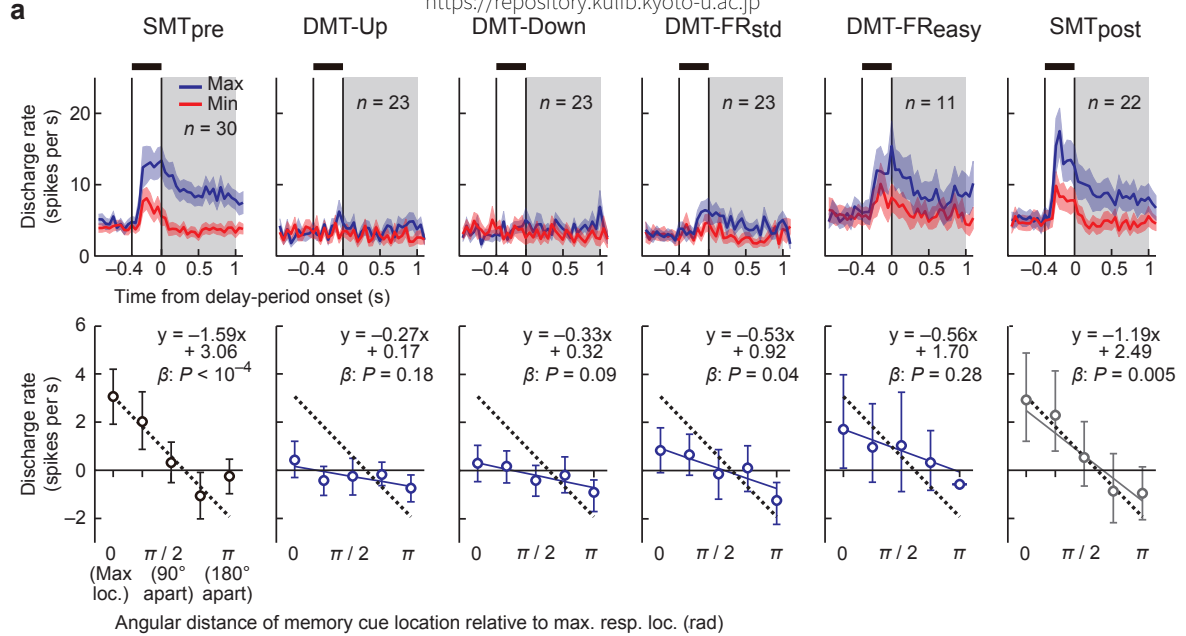

b
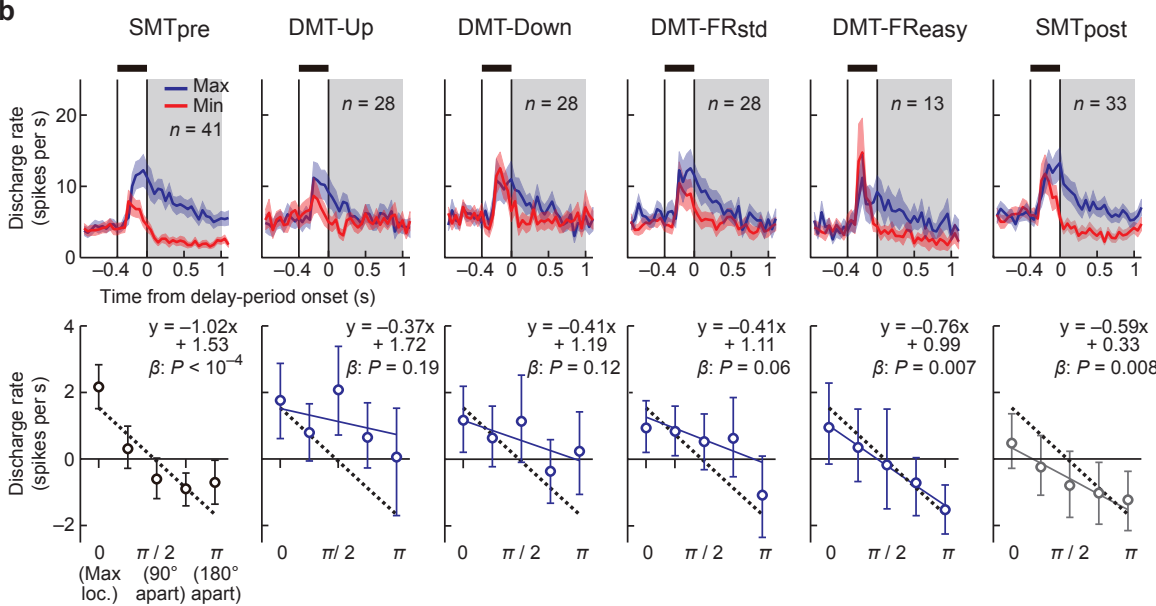

Angular distance of memory cue location relative to max. resp. loc. (rad)

Supplementary Figure 7 Population delay-period activities of individual monkeys. (a, b) Data are for monkeys S (a) and A (b). Upper row: population delay-period activity in the maximum (blue line) and minimum (red line) response locations across the six memory task conditions. In both monkeys, significant interaction effects [Task condition $\times$ Cue location] were observed among delay-period activity (monkey S: main effect of Task condition, $F_{5,126}=4.10, P=0.002$; Cue location, $F_{1,126}=66.05, P<10^{-4}$; interaction, $F_{5,126}=5.13$, $P=3 \times 10^{-4}$; monkey A: main effect of Task condition, $F_{5,165}=0.64, P=0.67$; Cue location, $F_{1,165}=65.64, P<$ $10^{-4}$; interaction, $F_{5,165}=3.77, P=0.003$ ). Bottom row: population spatial tuning during the delay-period. Conventions as in Fig. 5. In contrast to the cue-period activity, the degree of selectivity attenuation among delay-period activity was comparable between the two monkeys in the DMT conditions, suggesting that, in both monkeys, memory task processing was substantially disrupted in the later stage by the presence of the concurrent attention task. In accordance with this notion, behavioral results showed that, in both monkeys, prominent dual-task interference was observed in the trials that had long memory delay-period ( $>4.0 \mathrm{~s})$ (Supplementary Fig. 2a). The close correspondence between the individual variability among behavioral performance and that among response patterns of cue- and delay-period activities further supports the notion that the attenuation of neuronal selectivity for the memory cue location under DMT is a direct neural correlate of the behavioral cost of dual-task performance. 

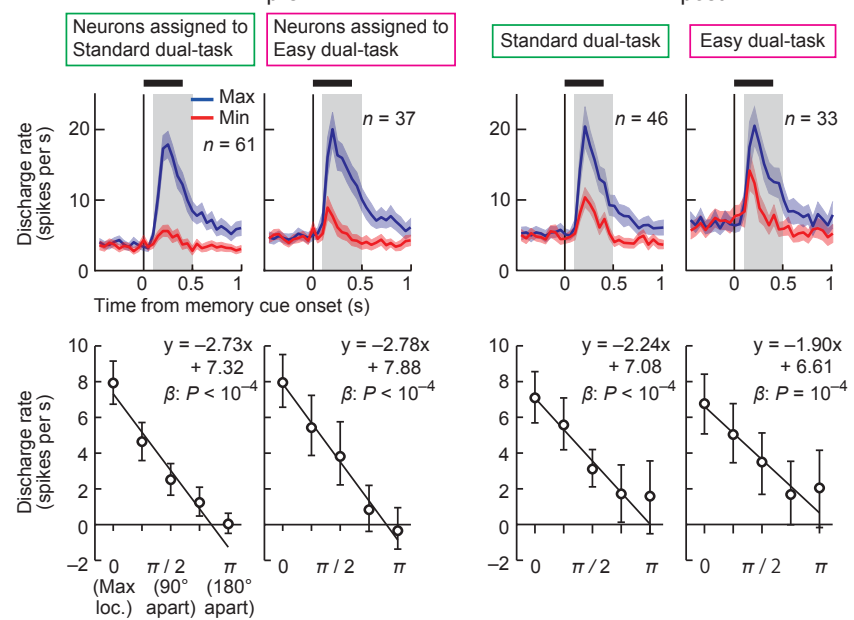

Angular distance of memory cue location relative to max. resp. location (rad)

C
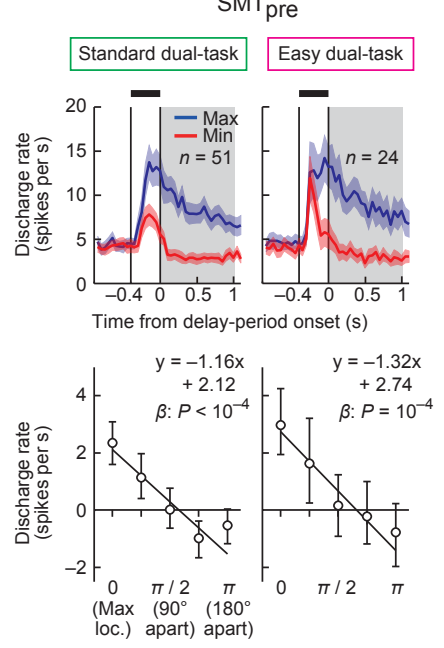

d
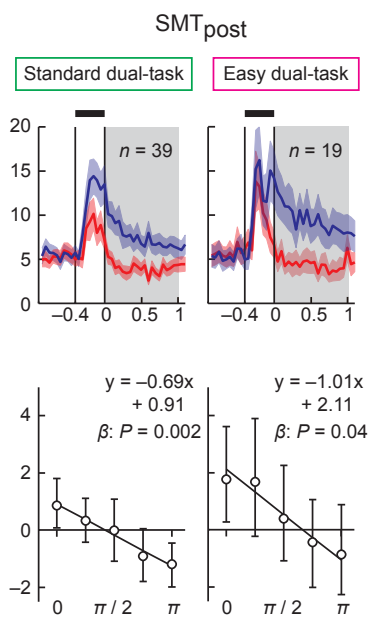

Angular distance of memory cue location relative to max. resp. location (rad)

Supplementary Figure 8 Comparison of cue- and delay-period activities in SMT between neurons assigned to the recording in the standard dual-task and the easy dual-task. (a,b) Upper row: population cue-period activities in the $\mathrm{SMT}_{\text {pre }}$ (a) and $\mathrm{SMT}_{\text {post }}$ (b) conditions for neurons assigned to the recording in the standard dual-task (left) and the easy dual-task (right). Conventions are the same as in Fig. 5. In both conditions, the activity patterns were highly similar between the groups of neurons assigned to the standard dual-task (left) and the easy dual-task (right) $\left(\mathrm{SMT}_{\text {pre }}\right.$ : main effect of Task assignment, $F_{1,96}=0.83, P=0.36$; Cue location, $F_{1,96}=$ 170.30, $P<10^{-4}$; interaction, $F_{1,96}=0.06, P=0.81$; $\mathrm{SMT}_{\text {post }}$ : main effect of Task assignment, $F_{1,77}=0.40, P=$ 0.53; Cue location, $F_{1,77}=67.03, P<10^{-4}$; interaction, $F_{1,77}=0.07, P=0.79$; two-way mixed-design ANOVA). Bottom row: population spatial tuning during the cue-period in $\operatorname{SMT}_{\text {pre }}$ (a) and $\mathrm{SMT}_{\text {post }}(\mathbf{b})$. In both $\mathrm{SMT}_{\text {pre }}$ and

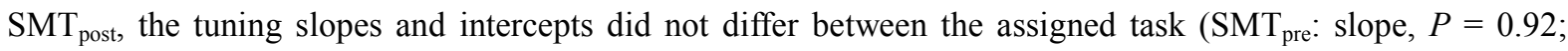
intercept, $P=0.44$; SMT $_{\text {post }}$ : slope, $P=0.56$; intercept, $\left.P=0.60\right)$. (c,d) Same as in (a) and (b), but for delay-period activity $\left(\mathrm{SMT}_{\text {pre }}\right.$ : main effect of Task assignment, $F_{1,73}=0.16, P=0.69$; Cue location, $F_{1,73}=92.18$, $P<10^{-4}$; interaction, $F_{1,73}=0.28, P=0.60$; $\mathrm{SMT}_{\text {post }}$ : main effect of Task assignment, $F_{1,56}=0.78, P=0.38$; Cue location, $F_{1,56}=43.21, P<10^{-4}$; interaction, $\left.F_{1,56}=1.54, P=0.22\right)$. The tuning slopes and intercepts did not differ between the assigned task $\left(\mathrm{SMT}_{\text {pre }}\right.$ : slope, $P=0.65$; intercept, $P=0.26$; $\mathrm{SMT}_{\text {post }}$ : slope, $P=0.49$; intercept, $P=0.10)$. 
a
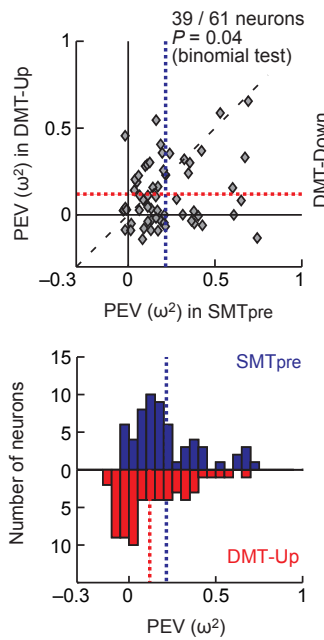

b
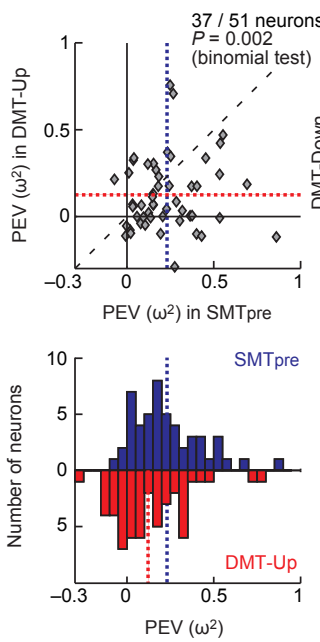

C

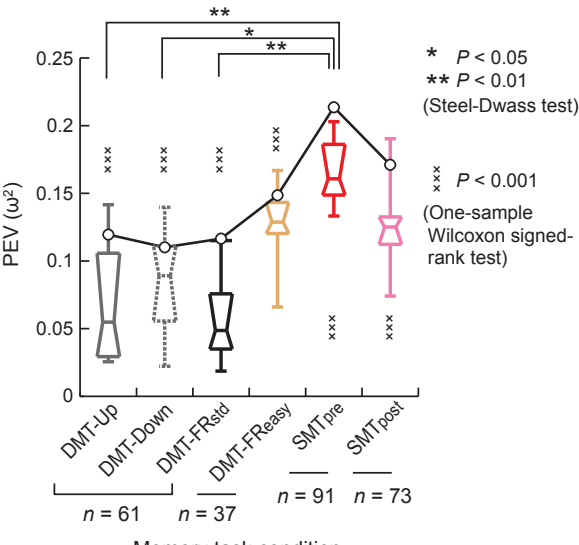

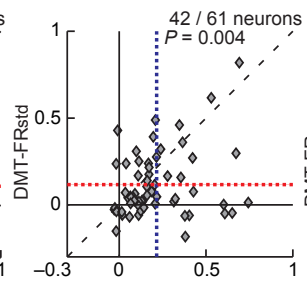

PEV $\left(\omega^{2}\right)$ in SMTpre

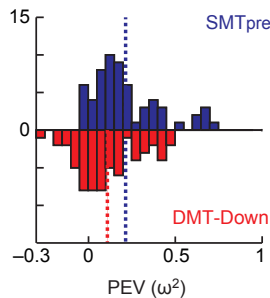

SMTpre
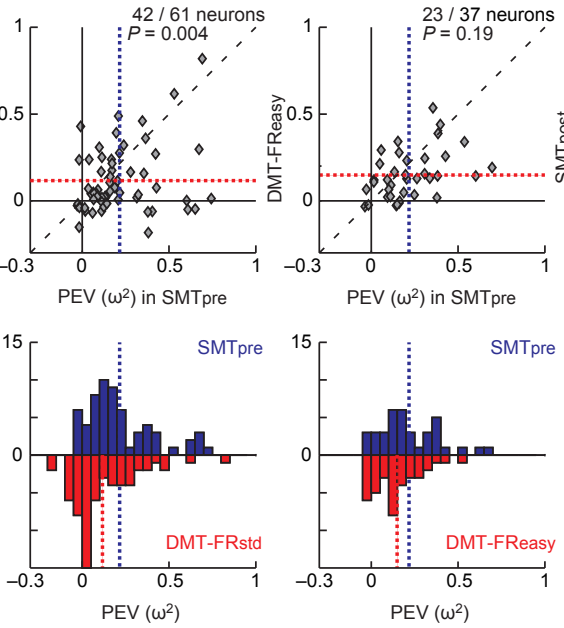

PEV $\left(\omega^{2}\right)$ in SMTpre

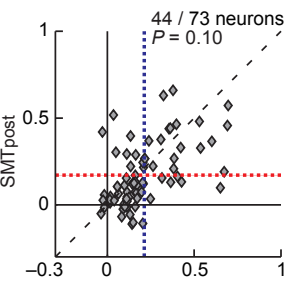

PEV $\left(\omega^{2}\right)$ in SMTpre
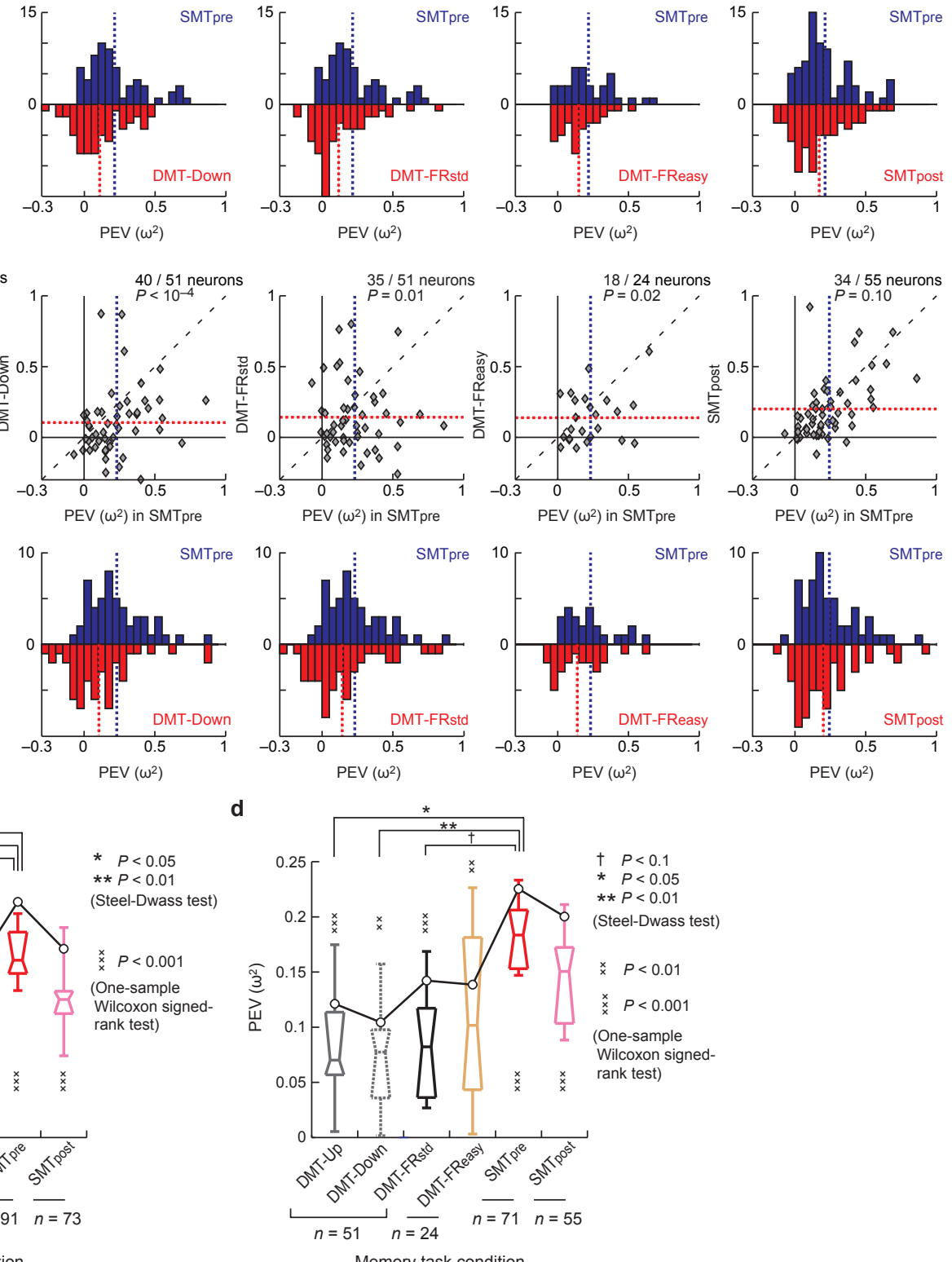

Memory task condition

Memory task condition 
Supplementary Figure 9 Comparison of single-neuron PEV values between the SMT and DMT conditions. (a) Upper row: scatter diagrams comparing PEV values of cue-period activity in $\mathrm{SMT}_{\text {pre }}$ to those in the four $\mathrm{DMT}$ and $\mathrm{SMT}_{\text {post }}$ conditions. Integration time window for PEV calculation was $0.4 \mathrm{~s}(0.1-0.5 \mathrm{~s}$ from memory cue onset). Blue dashed lines indicate the mean PEV values in $\mathrm{SMT}_{\text {pre }}$ Red dashed lines indicate the same neurons' mean PEV values in the corresponding conditions for comparison. Fractions show the number of

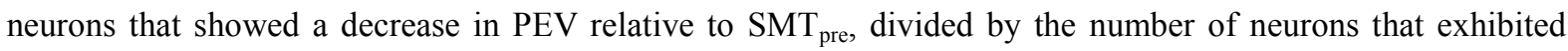

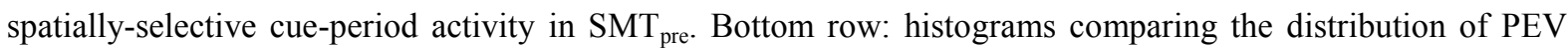
values between $\mathrm{SMT}_{\text {pre }}$ (blue bars) vs. each of the four DMT and $\mathrm{SMT}_{\text {post }}$ conditions (inverted red bars). (b) Same as in (a), but for delay-period activity. Integration time window was $1.0 \mathrm{~s}(0-1.0 \mathrm{~s}$ from memory cue

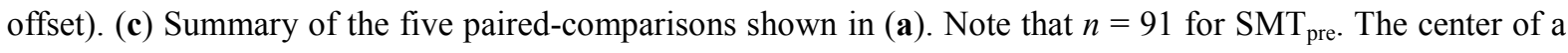
notched bar indicates the median value, edges are $\mathrm{CI}_{68 \%}$, and the error bar is the $\mathrm{CI}_{95 \%}$ of the median (bootstrap method). Open black circles indicate mean values. PEV values for cue-period activity were significantly different across memory task conditions (Kruskal-Wallis test, $P=4 \times 10^{-4}$ ), and $\mathrm{SMT}_{\text {pre }}$ showed a significantly

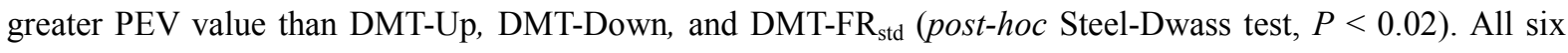
memory task conditions gave median PEV values significantly larger than zero (one-sample Wilcoxon signed-rank test). (d) Summary of the five paired-comparisons in (b). Note that $n=71$ for SMT pre. PEV values in delay-period activity were significantly different across memory task conditions (Kruskal-Wallis test, $P=$ 0.001). $\mathrm{SMT}_{\text {pre }}$ showed a significantly greater PEV value than DMT-Up, DMT-Down (post-hoc Steel-Dwass test, $P<0.03)$ and a substantially greater PEV value than $\mathrm{DMT}_{-\mathrm{FR}_{\text {std }}}(P=0.06)$. All six memory task conditions gave median PEV values significantly larger than zero. 
a
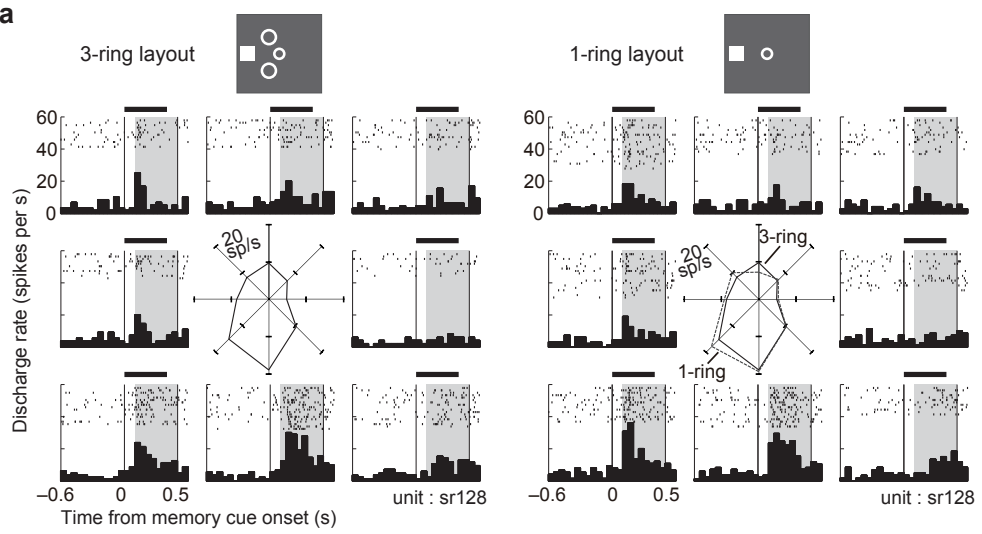

b
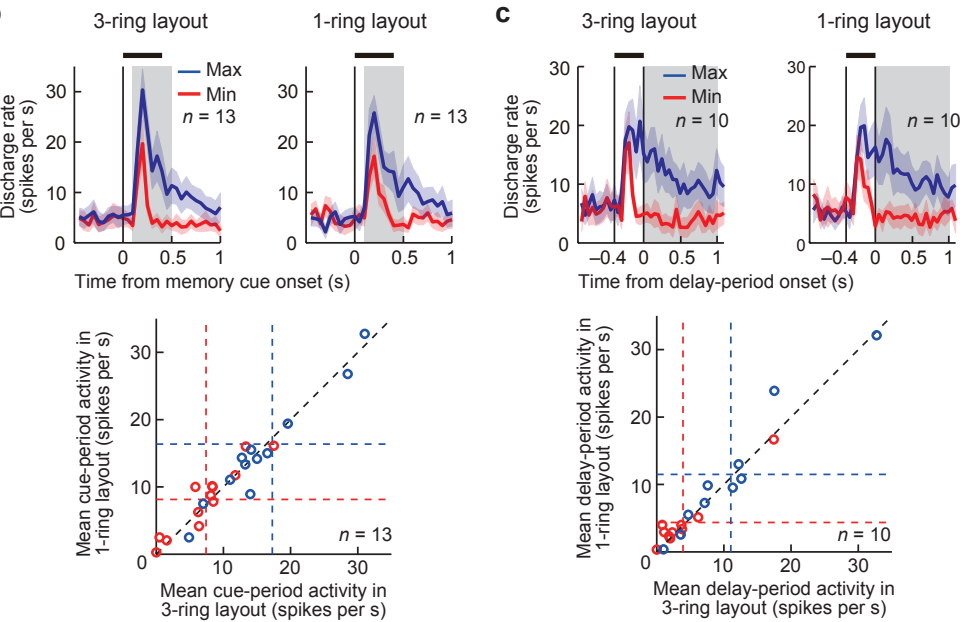

Supplementary Figure 10 Comparison of memory task-related activity between the 3-ring and 1-ring layout types in the modified single memory task. (a) Spatially-selective cue-period activity of a representative neuron that exhibited almost identical activities in the two layout types. Conventions as in Fig. 3. (b) Population activity in the 3-ring (top left) and 1-ring layout types (top right) for 13 spatially-selective cue neurons. A scatter diagram (bottom) shows a comparison of the strength of cue-period activity in the maximum (blue) and minimum (red) response locations that were selected from the five cue locations that were also used in DMT. Dotted lines indicate the mean cue-period activity across the population. The strength of cue-period activity was comparable between the two ring layout types at both the maximum $(P=0.31)$ and minimum $(P=0.19)$ response locations (Wilcoxon signed-rank test). (c) Same as in panel (b), but for 10 spatially-selective delay neurons. There was no significant difference in the strength of delay-period activity between the two layout types at both the maximum $(P=0.92)$ and minimum $(P=0.43)$ response locations. 

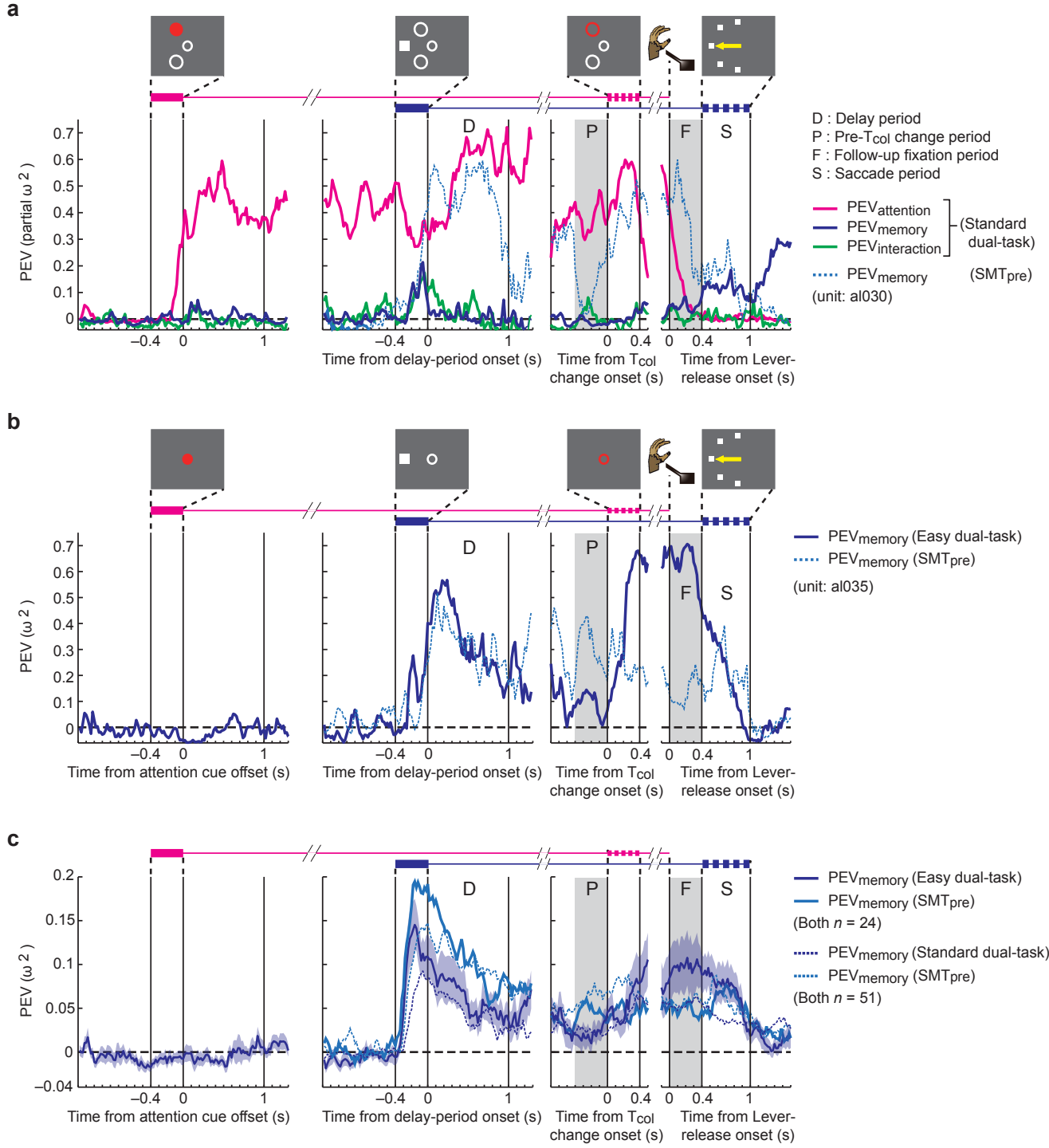

d
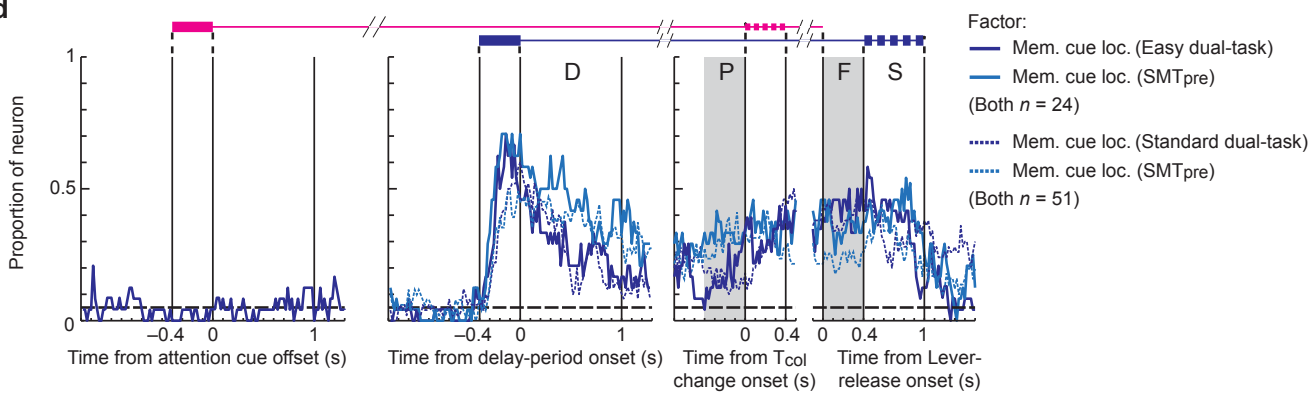
Supplementary Figure 11 Temporal dynamics of neuronal signals representing attention and memory task information in the standard and easy dual-tasks. (a) Time course of neuronal signals of an example neuron (the neuron shown in Supplementary Fig. 5b) representing the location of the attention cue $\left(\mathrm{PEV}_{\text {attention, }}\right.$ magenta),

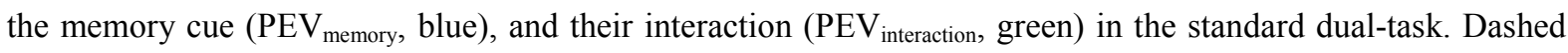
cyan line indicates the same neuron's $\mathrm{PEV}_{\text {memory }}$ in $\mathrm{SMT}_{\text {pre }}$ Conventions are the same as in Fig. 8a. (b) Time course of neuronal signals of an example neuron (the neuron shown in Supplementary Fig. 5c) representing the location of the memory cue $\left(\mathrm{PEV}_{\text {memory }}\right.$, blue) in the easy dual-task. Dashed cyan line indicates the same neuron's $\mathrm{PEV}_{\text {memory }}$ obtained in $\mathrm{SMT}_{\text {pre. }}$ (c) Population-averaged time course of $\mathrm{PEV}_{\text {memory }}$ in the easy dual-task (solid blue line, $n=24$ ). Shaded areas indicate s.e.m. The same neurons' population-averaged $\mathrm{PEV}_{\text {memory }}$ time series in $\mathrm{SMT}_{\text {pre }}$ are plotted as a solid cyan line. Dashed blue line and dashed cyan line indicate population-averaged $\mathrm{PEV}_{\text {memory }}$ time series in the standard dual-task and $\mathrm{SMT}_{\text {pre }}$, respectively for 51 neurons analyzed in Fig. 8 (the curves are the same as those shown in Fig. 8a). (d) Time course of the proportion of neurons that exhibited significant information $(P<0.05)$ about the memory cue location (solid blue line). The same neurons' results in $\mathrm{SMT}_{\text {pre }}$ are plotted as a solid cyan line. Dashed blue line and dashed cyan line indicate the results of the same analysis in the standard dual-task and $\mathrm{SMT}_{\text {pre }}$, respectively (both $n=51$, the curves are the same as in Fig. 8f). Horizontal dashed lines indicate the proportion expected by chance (5\%). 
a

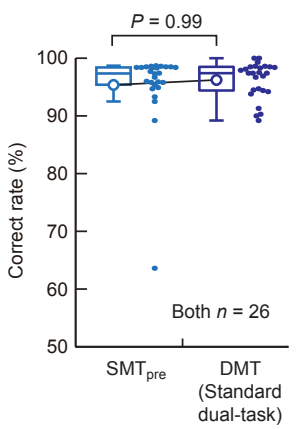

b

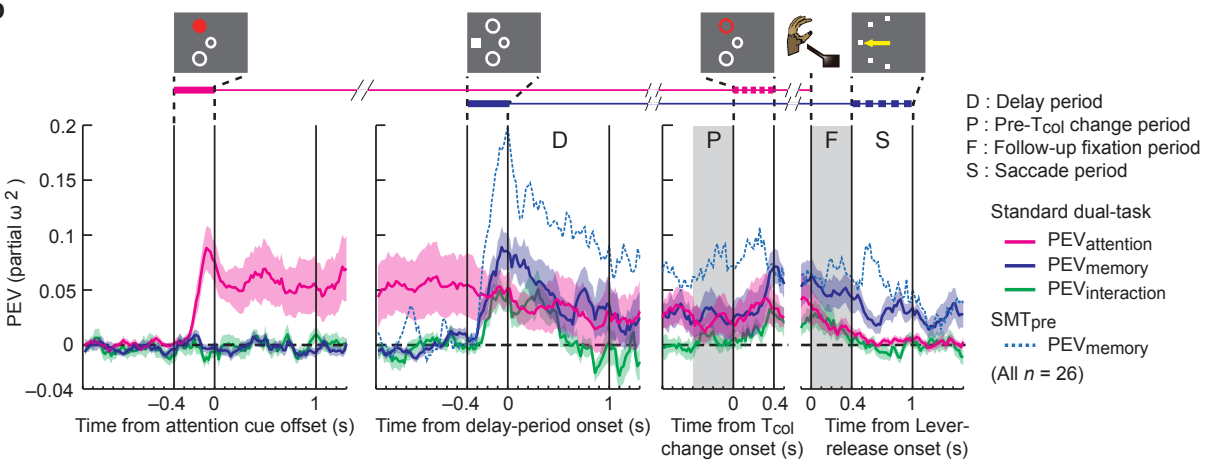

c

Memory task condition
PEV memory (standard dual-task) in

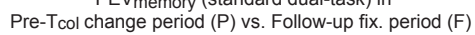

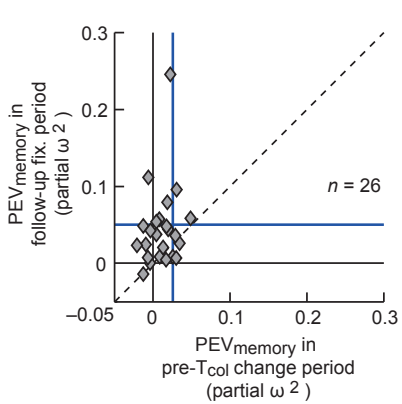

e

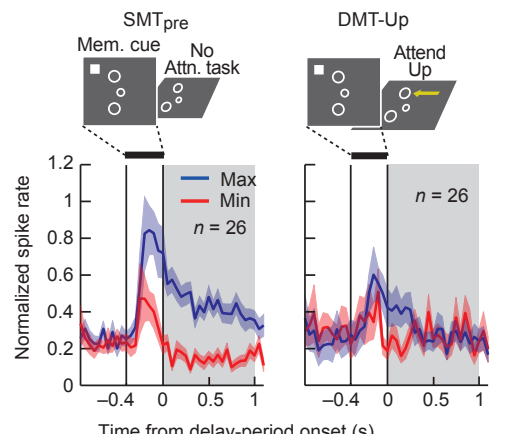

d PEV memory vs. PEV attention (standard dual-task) in Follow-up fixation period (F)
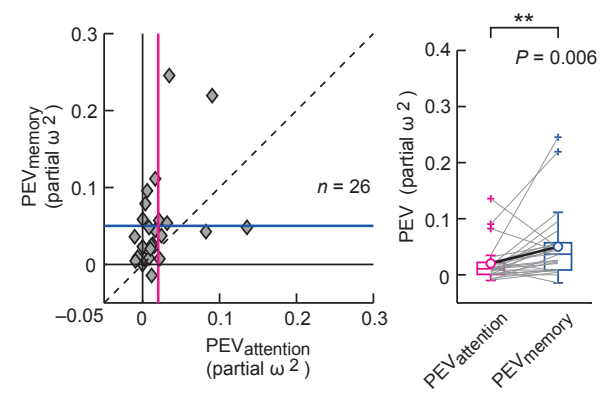

DMT-Down
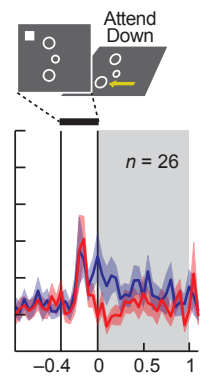
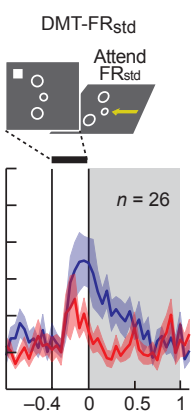

f

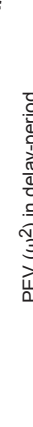

Kruskal Wallis test $X^{2}=9.31 ; P=0.03$

* $P<0.05$

(Post-hoc Steel-Dwass test)

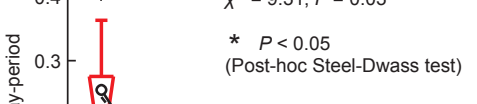

Memory task condition (all $n=26$ ) 
Supplementary Figure 12 Comparison of spatial selectivity between the SMT and DMT conditions after performance matching. (a) Behavioral performance matching across the SMT pre and DMT (standard dual-task) conditions. To achieve performance matching, session-by-session percent correct rates in each memory task condition were rank-ordered and split at the median. The bottom half of SMT pre sessions and the top half of DMT sessions were selected. This analysis included 51 sessions where spatially-selective delay-period activity was recorded in $\mathrm{SMT}_{\text {pre. }}$. Data from the three DMT conditions in the standard dual-task (DMT-Up, DMT-Down and DMT-FR ${ }_{\text {std }}$ ) were collapsed. The sessions from the individual monkey were separately rank-ordered to avoid a biased subsampling from one monkey. The subsampled sessions gave highly similar percent correct

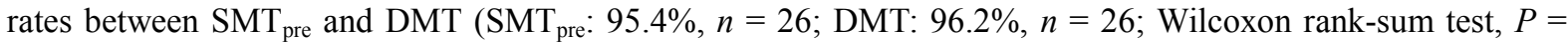
0.99). Conventions as in Fig. 2c. (b) Time course of $\mathrm{PEV}_{\text {attention }}$ (magenta), $\mathrm{PEV}_{\text {memory }}$ (blue), and $P E V_{\text {interaction }}$ (green) in the standard dual-task for the 26 subsampled sessions. The magnitude of $\mathrm{PEV}_{\text {memory }}$ during the delay-period $(D)$ was significantly attenuated relative to that in $\operatorname{SMT}_{\text {pre }}(n=26$, dashed cyan line) (Wilcoxon rank-sum test, $P=0.03$ ). Conventions as in Fig. 8. (c) Comparison of $P E V_{\text {memory }}$ between the pre- $\mathrm{T}_{\text {col }}$ change period and the follow-up fixation period in the standard dual-task. (d) Comparison of PEV $\mathrm{Pemory}_{\text {and }}$ PEV attention during the follow-up fixation period in the standard dual-task. In the subsampled DMT sessions that gave

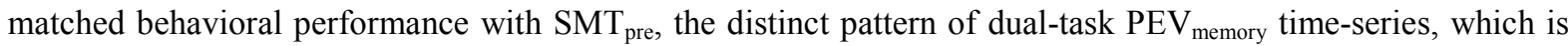
characterized by initial attenuation and subsequent reawakening, was still present. Therefore, these neuronal dual-task effects are inconsistent with the explanation of general, task-independent effect of task-difficulty. Rather, the present effects are directly attributable to the presence of the demands for the concurrent attention task processing, and thus unique to dual-task performance. (e) Normalized population-averaged delay-period activity (grey shaded area) in the maximum (blue line) and minimum (red line) response locations in $\mathrm{SMT}_{\text {pre }}$ and the three DMT conditions in the standard dual-task for the subsampled sessions. For comparing delay-period activity across the four conditions, behavioral performance in DMT-Up, DMT-Down and DMT-FR $_{\text {std }}$ were rank-ordered separately. The subsampled sessions gave similar percent correct rates across the four conditions ( $P=0.14, n=26$ for each of the four conditions, $)$. For each neuron, firing rate in each 50 -ms bin was divided by the peak delay-period firing rate at the maximum response location in the $\mathrm{SMT}_{\text {pre }}$ condition. Compared with $\mathrm{SMT}_{\text {pre, }}$ the difference in activity between the maximum and minimum response locations was

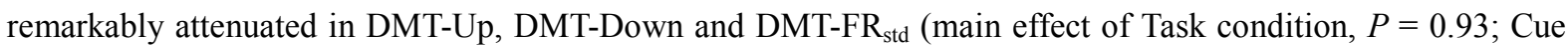
location, $P<10^{-4}$; interaction, $P=6 \times 10^{-4}$; two-way mixed design ANOVA). Conventions as in Fig. 5c. (f) Comparison of PEV values of the delay-period activity between the SMT and three DMT conditions. The three DMT conditions in the standard dual-task exhibited attenuation in spatial selectivity relative to $\mathrm{SMT}_{\text {pre }}$ (Kruskal-Wallis test, $P=0.03$ ). Conventions as in Supplementary Fig. 9d. Similar result was obtained when the rank-order of sessions was done over monkey-collapsed data (dotted line, $P=0.03$ ). 